Dr. J. A. Swaffield

Service Systems Program

Center for Building Technology

U.S. Department of Commerce

National Bureau of Standards

Washington, DC 20234

and

Drainage Research Group

Department of Building Technology

Brunnel University

U.K.

June 1981
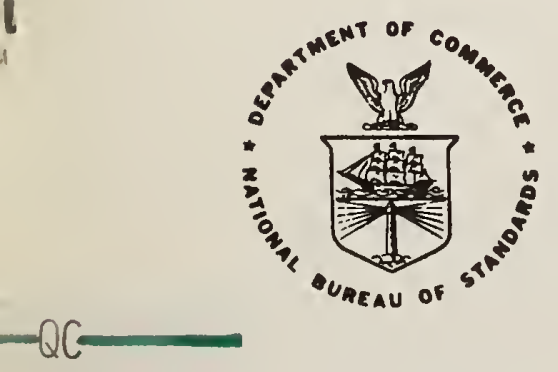



\section{ENTRY TRANSITION WATER SURFACE PROFILE PREDICTION IN SUPERCRITICAL PARTIALLY FILLED PIPE FLOW}

Dr. J. A. Swaffield

Service Systems Program

Center for Building Technology U.S. Department of Commerce National Bureau of Standards

Washington, DC 20234

and

Drainage Research Group

Department of Building Technology Brunnel University

U.K.

June 1981

U.S. DEPARTMENT OF COMMERCE, Malcolm Baldrige, Secretary NATIONAL BUREAU OF STANDARDS, Ernest Ambler, Director 



\section{PREFACE}

This report is one of a group documenting National Bureau of Standards (NBS) research and analysis efforts in developing water conservation test methods, models for technical and economic analysis, and strategies for implementation and acceptance of practices. This work is sponsored by the Department of Housing and Urban Development, Office of Policy Development and Research, Building Technology and Standards Division, under HUD Interagency Agreement H-48-78. 


\section{SUMMARY}

The criteria governing the development of steady partially filled supercritical pipe.flow are presented together with the necessary techniques to determine the water surface profile in the pipe entry transition length.

The establishment of full bore flow is predicted for a range of flow rates and pipe design parameters. Based on the water surface profile calculation technique pipe length predictions are presented to avoid the air pressure fluctuations in the drainage system that result from full bore flow establishment.

Tabular data are presented to allow design decisions to be made that link pipe slope, diameter and roughness to the need to avoid full bore flow. A graphical technique is also presented that removes the necessity to interpolate from the tabular data.

The effect of entry geometry loss coefficients is included in the techniques presented. 


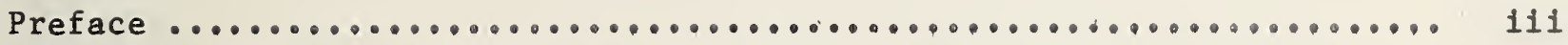

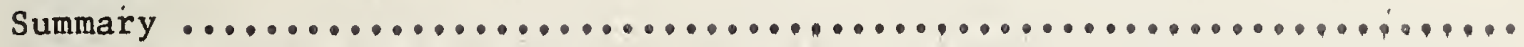

List of Figures and Tables $\ldots \ldots \ldots \ldots \ldots \ldots \ldots \ldots \ldots \ldots \ldots \ldots \ldots \ldots \ldots \ldots$

Notation

1. INTRODUCTION $\ldots \ldots \ldots \ldots \ldots \ldots \ldots \ldots \ldots \ldots \ldots \ldots \ldots \ldots \ldots \ldots \ldots \ldots \ldots \ldots \ldots \ldots \ldots \ldots \ldots \ldots$ 1

2. THEORETICAL CONSIDERATIONS .............................. 2

2.1 Steady Uniform Flow in Partially Filled Pipe Flow ........... 2

2.2 Entry Transition Length in Partially Filled Pipe Flow ........ 3

2.3 Gradually Varied Flow in Partially Filled Pipes ............. 5

2.4 Loss Coefficients for Slope Transitions in Partially Filled

Plpe Flow ...................................... 6

3. CALCULATION TECHNIQUES AND PRESENTATION OF RESULTS ............ 8

3.1 Determination of Normal and Critical Depths .............. 8

3.2 Numerical Integration for Surface Profiles ................. 8

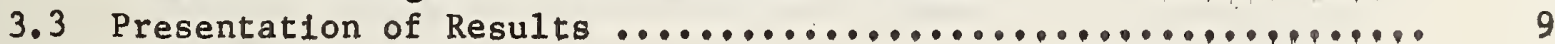

3.4 Input Data Choice .................................. 9

4. DISCUSSION OF RESULTS ................................ 10

5. CONCLUSIONS $\ldots \ldots \ldots \ldots \ldots \ldots \ldots \ldots \ldots \ldots \ldots \ldots \ldots \ldots \ldots \ldots \ldots \ldots \ldots \ldots \ldots \ldots \ldots \ldots$

6. REFERENCES ........................................... 14

APPENDIX 1 Tabular Data Relating Flow Normal Depth and Transition

Length to Flow and Pipe Parameters .................. 26

APPENDIX 2 Description of Program PROFIL $2 \ldots \ldots \ldots \ldots \ldots \ldots \ldots . . . . . . .91$ 
Figures

1 Derivation of Chezy's equation for free surface flow ....... 15

2 Relationship between flow specific energy and flow depth ..... 16

3 Transition length downstream of a pipe slope change in partially filled pipe flow ........................ 17

4 Basis of gradually varied flow analysis ............... 18

5 Schematic representation of numerical integration to determine

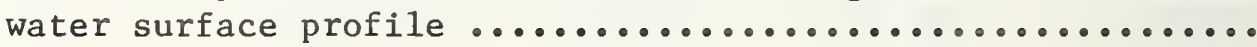

6 Water surface profiles for a range of pipe gradients, illustrating increase in transition length as pipe slope is increased 00000000000000000000000000000000000000000000000000

7 Water surface profile for a range of pipe roughness values, illustrating increased transition length as pipe roughness is decreased

8 Flow normal depth as a function of pipe and flow parameters

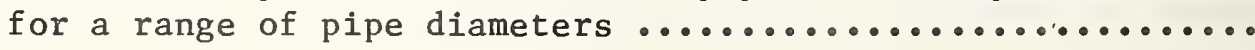

9 Entry depth as a function of flow rate and entry specific energy .....................................

Transition length as a function of depth change from entry to normal depth, note that flow rate and entry energy are. subsumed in the depth change term

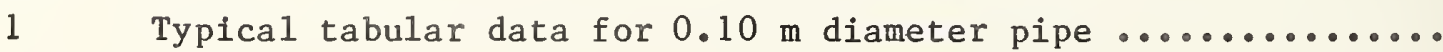



A Channel cross sectional area
C Chezy constant
D Pipe diameter
E Specific energy
g Acceleration due to gravity
h Flow depth
$\mathrm{h}_{\mathrm{c}} \quad$ Critical flow depth
$h_{e} \quad$ Entry flow depth
$\mathrm{h}_{\mathrm{n}} \quad$ Normal flow depth
L Transition length
m Hydraulic mean depth
n Manning coefficient
P Wetted perimeter
Q Flow rate
S Slope of energy grade line
So Channel slope
$\mathrm{T}$ Water surface width in channel
V Local average velocity
$x \quad$ Axial flow direction
Z Elevation channel above some datum
p Fluid density
$\theta_{1} \quad$ Approach pipe slope
$\theta_{2} \quad$ Test pipe slope 



\section{INTRODUCTION}

In the design of bullding dralnage systems the maintenance of appllance trap seals is a major criterion. Trap seal depletion may occur as a result of either negative or positive alr pressure transients in the drainage pipework. Poa1tive pressures may be generated if the water flow in the syatem becomes full bore at some downstream location. The most common cause of such a closure of the air passage above the water surface is the flow depth change that accom panies a change in pipe slope. The transition from vertical stack flow to the near horizontal building drain, commonly set at gradients in the range $1 / 40$ to $1 / 80$, results in a rapid depth increase downstream of the slope change that may result in the establishment of local full bore flow. Transition from the entry depth to a greater depth can be erroneously interpreted in experimental observations as an hydraulic jump; the theoretical predictions developed here indicate that a monotonically increasing (smooth) depth change can occur. The conditions under which a hydraulic jump exist are discussed in an earlier study [1].

The design criteria necessary to avoid this condition are set out in this report, together with the necessary computing techniques and design table examples for a range of typical pipe diameters, gradients and flow rates. 


\section{THEORETICAL CONSIDERATIONS}

\subsection{STEADY, UNIFORM FLOW IN PARTIALLY FILLED PIPE FLOW}

Figure 1 illustrates the force balance equation for steady flow in an open channel or partially filled duct. The common expression of this relationship is known as the Chezy equation where

$$
\begin{aligned}
& \mathrm{V}=\mathrm{C} \sqrt{\mathrm{m} \mathrm{S}_{\mathrm{O}}} \\
& \mathrm{m}=\text { hydraulic mean depth } \mathrm{A} / \mathrm{P}, \mathrm{m} \\
& \mathrm{S}_{\mathrm{O}}=\sin \Theta, \text { duct slope } \\
& \mathrm{V}=\text { mean velocity, } \mathrm{m} / \mathrm{s} \\
& \mathrm{C}=\text { Chezy constant. }
\end{aligned}
$$

The value of loss coefficient $C$ was found by Manning to be dependent on hydraulic mean depth and duct surface roughness $n$. The Manning formula is the simplest of the open channel equations:

$$
\begin{aligned}
& \mathrm{V}=\mathrm{m}_{\mathrm{n}} \mathrm{m}_{\mathrm{o}}^{2 / 3} \mathrm{~s}_{\mathrm{o}}^{1 / 2} \\
& \mathrm{Q}=1 \mathrm{~A} \mathrm{~m}^{2 / 3} \mathrm{~s}_{\mathrm{o}}^{1 / 2}
\end{aligned}
$$

where $Q$ is the flow rate $\mathrm{m}^{3} / \mathrm{s}$

$A$ is the flow cross sectional area, $\mathrm{m}^{2}$

The value of the Manning coefficient, $n$, varies with pipe or channel material. Chow [2] suggests values in the range 0.009 to 0.020 for materials commonly found in building drainage systems. The utilization of $\mathrm{n}$ as a variable with depth can be introduced (in the computer program) for more detailed calculations. The effects of wall accumulations on $\mathrm{n}$ as a function of deposited materials is unknown.

Equation 1 effectively determines the flow depth, $h$, under steady, uniform conditions, only one value of $h$ yielding the values of $A$ and $m$ necessary to satisfy the equation. As this depth is by definition constant downstream, $\mathrm{dh} / \mathrm{dx}=0$, it must also be the terminal depth corresponding to the flow terminal velocity at that channel slope.

This depth, $h_{n}$, is commonly referred to as the normal depth.

The specific energy of the flow may be defined as

$$
E=h+v^{2}
$$

where $\mathrm{h}=$ local flow depth, $\mathrm{m}$

$\mathrm{V}=$ local average flow velocity, $\mathrm{m} / \mathrm{s}$ 
Figure 2 illustrates the alternate depths that w111 satisfy equation ( 3 ) and their significance in terms of the flow definition.

From equation ( 3 ) and figure 2 it may also be seen that the flow specific energy has a minimum value below which the given flow conditions cannot exist. In a general, non rectangular channel this value may be determined:

$$
\begin{aligned}
& E=h+\underset{2 A^{2}}{Q^{2}} \\
& \frac{d E}{d h}=0=1-\frac{Q^{2}}{g A^{3}} \frac{d A}{d h}
\end{aligned}
$$

From figure 3

$$
\mathrm{dA}=\mathrm{T} \cdot \mathrm{dh}
$$

where $T$ is the surface width at any depth, $h$.

From equations (4) and (5) the minimum value of $E$ will occur at a depth value, $h_{c}$, that satisfies the expression

$$
1-Q^{2} T / g A^{3}=0
$$

This value of $h$ is referred to as the flow critical depth $h_{c}$.

If the normal flow depth $h_{n}$ exceeds $h_{c}$ then the terminal flow would be termed subcritical, or tranquil flow. If $h_{n}$ is less than $h_{c}$ then the flow is termed rapid or supercritical.

It should be stressed that $h_{c}$ is independent of plpe slope and plpe surface roughness; while the normal depth is dependent on both. Thus the same volume flow rate in any particular pipe may be rapid or tranquil depending on pipe slope, and similarly the same flow rate in a serles of constant diameter pipes will be tranquil or rapid depending on roughness.

Pipes or channels in which rapid flow is normal are termed steep, pipes or channels in which tranquil flow is normal are termed of mild slope.

\subsection{ENTRY TRANSITION LENGTH IN PARTIALLY FILLED PIPE FLOW}

It will be seen from figure 2 that the flow depth at any point along a partially filled drain is dependent on both flow rate and specific energy. However the only stable depth is that represented by the normal flow equation, (2), thus a transition region may be expected in any partially filled pipe flow when the inlet flow conditions, expressed in terms of $Q$ and $E$, do not match the normal depth characteristic values.

At the base of a vertical stack it is unlikely that the flow rate and specific energy will match the downstream drain normal depth values. In general the specific energy at the drain inlet will be higher than the normal depth and 
flow characteristic specific energy, so that the entry flow depth will be less than the downstream normal depth.

Under, these conditions frictional forces acting on the fluid flow result in an increase in flow depth downstream until the flow normal depth is reached. Thus a transition regime may be identified whose length is dependent on the pipe slope, diameter and roughness and the entry flow rate and specific energy. Figure 3 illustrates this transition region. This description is generally true whether the flow downstream is classified as subcritical or supercritical.

For the subcritical flow case, the normal depth to be achieved is greater than the flow critical depth and hence its establishment requires the generation of a hydraulic jump. This topic was dealt with in an earlier report [1].

For the supercritical flow case the depth profile may increase downstream until the normal depth is achieved without the generation of a hydraulic jump, or local flow depth discontinuity.

It is however necessary to distinguish clearly between the generation of local full bore flow due to a hydraulic jump and the observation of full bore flow as a result of a "smooth" transition to normal depth in supercritical flow. Comparison of the normal depth, calculated from equation (2), to the critical depth, as calculated from equation (6), is sufficient to determine whether a jump will occur, or whether it is sufficient to only consider the developing supercritical flow depth profile.

Care should be taken in any literature survey of drainage studies in this area as the term "hydraulic jump" is often used loosely to identify both the true jump condition and the establishment of full bore flow as a result of the supercritical depth transition described above.

In both cases the likelihood of full bore flow is predictable from a comparison of the target normal depth with the pipe diameter.

In the analysis presented steady flow conditions are assumed at a range of flow rates. In practice the entry flow to the drain will follow some flow vs time profile. Thus the establishment of full bore flow will depend on the peak entry flow rate and its attenuation as the inflow surge progresses down the pipe. A study of attenuation in supercritical free surface flow [2] indicates that the effect will be small over the first 5 to $6 \mathrm{~m}$ of drain so that it is reasonable to base design tables on the assumption that the entry profile may be represented by a series of increasing steady flow rates. Adjustments for the flow increase to permit adjustment for the series of stepwise increased rates may be made in the integrands of the equations in this report.

In order to provide design data two calculation techniques are required:

(1) Comparison of flow normal depth with both the drain critical depth value to determine the applicable flow regime, and the pipe diameter, to determine whether full bore flow is possible at this combination of flow rate, pipe diameter, gradient and roughness. 
(2) Calculation of the water surface profile from the pipe entry downstream to either the establishment of normal depth flow or full bore flow. The necessary equations for ( 1 ) above have been established. The calcularlon of the water surface profile requires the use of gradually varied flow analysis.

\subsection{GRADUALLY VARIED FLOW IN PARTIALLY FILLED PIPES}

Gradually varied flow is steady. non-uniform flow of a special type. The flow parameters are assumed to change slowly, if at all, in the flow direction. The basic assumption in the treatment of this type of flow is that the local head loss at any section is given by the Manning expression, (2), for the 1dentical local flow depth and rate under assumed steady, uniform flow conditions.

Depth profile predictions by numerical integration are based on this assumption, expressed in terms of figure 4 by

$$
d L\left\{\frac{V^{2}}{2 g}+\left(z_{O}-S_{O} L\right)+h\right\}=-\left\{\underset{\operatorname{Am}^{2 / 3}}{n Q}\right\}^{2}
$$

where $\left(Z_{O}-S_{O} L\right)$ is the elevation at distance $L$ along the channel, measured in the downstream direction; $S_{0}$ is $\sin \theta$, channel bed slope,

hence $-\frac{\mathrm{V}}{\mathrm{g}} \frac{\mathrm{dV}}{\mathrm{dL}}+\mathrm{s}_{0}-\frac{\mathrm{dh}}{\mathrm{dL}}=\left(\begin{array}{c}\mathrm{nQ} \\ \mathrm{Am}\end{array}\right.$

and as, $Q=V A$

$$
\frac{d V}{d L} A+V \frac{d A}{d L}=0
$$

and as $\frac{d A}{d h}=T$ from equation 5 it follows that

$$
\frac{d V}{d L}=\frac{V}{A} \frac{d A}{d L}=-\frac{V T}{A} \frac{d h}{d L}=-\frac{Q T}{A^{2}} \frac{d h}{d L}
$$

and substituting in equation (8) yields

$$
\begin{aligned}
& Q^{2} \mathrm{~T} d \mathrm{dh}+\mathrm{S}_{\mathrm{O}}-\underset{\mathrm{dL}}{\mathrm{dL}}=\left\{\begin{array}{c}
\mathrm{n} \mathrm{Q}_{\mathrm{Am}}^{2 / 3} \\
\mathrm{AA}^{3} \mathrm{dL}
\end{array}\right\}^{2} \\
& \mathrm{dL}=\left\{\begin{array}{c}
1-\mathrm{Q}^{2} \mathrm{~T} / \mathrm{g} \mathrm{A}^{3} \\
\mathrm{~s}_{0}-\left(\mathrm{nQ} / \mathrm{Am}^{2 / 3}\right)
\end{array}\right\} \mathrm{dh}
\end{aligned}
$$

or 


$$
L=\int_{h_{0}}^{h_{1}} S_{0}-\left(n Q / A m^{2 / 3}\right)^{2} d h
$$

where' $\mathrm{L}$ is the distance between two known depths $\mathrm{h}_{\mathrm{O}}, \mathrm{h}_{\mathrm{l}}$.

Figure 5 illustrates this numerical integration, which may be conveniently achieved by Simpson's rule.

The numerator and denominator of equation (10) will be recognized as the equations determining the critical and normal flow depths in an open channel.

When the term $\left(1-\mathrm{Q}^{2} \mathrm{~T} / \mathrm{gA}^{3}\right)$ is zero the flow is at critical depth, i.e., there is no change in $\mathrm{L}$ for a change in $\mathrm{h}$.

For uniform cross section channels with constant roughness, $\mathrm{n}$, and slope, $\mathrm{S}_{\mathrm{O}}$, the expression (10) becomes solely a function of flow depth $h$.

In order to numerically evaluate (10) it is necessary to define boundary conditions from which the integration may proceed. It should be stressed that the integration may be carried out either upstream or downstream from a known depth point. This ability is central to the use of this technique to determine the position of a profile continuity, such as a hydraulic jump.

Figure 5 illustrates the control depths used in the prediction of the water surface profiles in the case being investigated, namely the change in slope of an open channel.

If the flow rate $Q$ and specific energy are known at pipe entry, at $B$, figure 3 then the depth at $B$ may be calculated by choosing the lower depth root of equation (4). In the study reported a range of entry specific energy values for a constant inflow were obtained by considering the entry flow at $B$ to have attained terminal flow conditions in an approach pipe, $A B$ figure 3, set at a range of gradients from $15^{\circ}$ to $90^{\circ}$, however this artifice is not strictly necessary as any suitable energy values could have been utilized.

The choice of dh values in the numerical integration is based on the difference between the control depth at entry and the "target" depth, representing the normal flow depth to be achieved downstream.

For the example in figure 5 the dh value is

$$
\mathrm{dh}=\left(\mathrm{h}_{\mathrm{n}}-\mathrm{h}_{\mathrm{e}}\right) / \mathrm{N}
$$

where $\mathrm{N}$ is a reasonable number in the range 10-30. Since the change from $\mathrm{h}_{\mathrm{e}}$ to $h_{n}$ can be expected to fall within an order of magnitude and the monotonic change (except for the jump condition) in the function h(L) is not rapid the size of incremental steps can be of the order of unity. 
If the normal depth, $h_{n}$, exceeds the pipe dlameter. $D$, then the numerical integration is terminated when the predicted flow depth exceeds the pipe diameter value.

\subsection{LOSS COEFFICIENTS FOR SLOPE TRANSITIONS IN PARTIALEY FILLED PIPE FLOW}

No data could be obtained on the loss coefflcients for slope transitions in open channel flow. For this reason the results presented assume no loss at the pipe entry. The computer program as written has been designed to include such a loss coefficient, in the range 0 to 1 , should such data become avallable from a future experimental program. The effect of such a loss would be to increase the flow depth at pipe entry, with a consequent decrease in the kinetic energy term at pipe entry. In turn this would have the effect of generally moving the energy transition upstream towards pipe entry. Experimental work is required as a back up to the computer simulation to clarify this area. 


\section{CALCULATION TECHNIQUES AND PRESENTATION OF RESULTS}

\subsection{DETERMINATION OF NORMAL AND CRITICAL DEPTHS}

The bisection method was used to solve the equation defining both critical flow depth

$$
X=1-Q^{2} \mathrm{~T} / \mathrm{gA}^{3}
$$

and normal flow depth

$$
\mathrm{Y}=\mathrm{S}_{\mathrm{O}}-\left(\mathrm{n} \mathrm{Q} / \mathrm{Am}^{2 / 3}\right)^{2}
$$

It may be assumed that both $\mathrm{X}$ and $\mathrm{Y}$ have zero values for some value of depth $\mathrm{h}$ in the range $0<\mathrm{h}<\mathrm{D}$ for pipe case or $0<\mathrm{h}<\mathrm{W}$ for the square section case.

This initial interval is bisected and $h=\mathrm{D} / 2$ or $\mathrm{w} / 2 \mathrm{for}$ the square section) used to evaluate $\mathrm{X}, \mathrm{Y}$. If the resulting values are positive then the root is less than the midpoint. The upper limit is then reset equal to the $h$ value just used and the remaining interval bisected. The process repeats until a root is obtained. If the $\mathrm{X}$ or $\mathrm{Y}$ value had been negative then the root would be greater than the trial $\mathrm{h}$ value. In this case the lower limit is reset to the trial $h$ value and incremented with the remaining interval bisected.

Due to the need to include the area depth relationship this solution must be undertaken by an iterative process. The computer time taken depends on the complexity of the area-depth function.

\subsection{NUMERICAL INTEGRATION FOR SURFACE PROFILES}

The integration of the position vs depth profile

$$
L=\int_{h_{0}}^{h_{1}} S_{0}-\left(n Q / A m^{2 / 3}\right)^{2} d h
$$

$\mathrm{h}_{1}$

is achieved by means of Simpson's Rule. Let the integral $\mathrm{X}=\int \mathrm{F}(\mathrm{h}) \mathrm{dh}$, ho

then if the interval $h_{1}-h_{0}$ is divided into 2 equal increments, the value of $X$ is given by

$$
X=\frac{1}{3} d h\left[F\left(h_{0}\right)+4 F\left(h_{0}+d h\right)+F\left(h_{0}+2 d h\right)\right]
$$

As the integration moves on the length traversed may be accumulated as $\mathrm{L}=$ $\mathrm{L}+\mathrm{X}$ at the completion of each integration. 


\section{3 PRESENTATION OF RESULTS}

The transition profiles for the following cases are presented in tabular form in Appendix I:

Flow rates 2 to $12 \mathrm{l} / \mathrm{s}$, extended to $22 \mathrm{l} / \mathrm{s}$ for the $0.15 \mathrm{~m}$ diameter pipe case

Pipe diameters: $0.075 \mathrm{~m}, 0.10 \mathrm{~m}$ and $0.15 \mathrm{~m}$

Roughness coefficients: $0.009,0.012,0.015,0.018$

Pipe gradients: $1 / 20,1 / 40,1 / 60,1 / 80$

Entry specific energy range simulated by varying the approach pipe slope from $15^{\circ}$ to $90^{\circ}$.

In addition to tabular data, an alternative graphical technique is presented.

\subsection{INPUT DATA CHOICE}

As far as possible the choice of input test conditions was governed by the range of values likely to be found in drainage systems. The pipe diameters chosen, $0.075,0.10$ and 0.15 conform to this criterion as do the pipe gradients used for all test cases, $1 / 40$ to $1 / 80$. The choice of pipe roughness or Manning coefficient was more difficult, however values in the range 0.009 to 0.015 are recommended in many texts, i.e. Jaeger [3] and Chow [4].

Losses at the change of slope that produces the conditions conducive to full bore flow have been ignored in this treatment. No available data on open channel transition loss coefficients for partially filled pipes or channels could be obtained. The program is capable of dealing with transition losses however via an input data control variable provided the loss can be expressed as a factor, 0 to 1.0 , of the specific energy of the flow at pipe entry. 


\section{DISCUSSION OF RESULTS}

The water surface profile integration downstream from pipe entry is illustrated by figures 6 and 7 . It will be seen that the transition length, or the distance to full bore flow or normal depth, whichever is less, depends on both the flow rate and entry specific energy and hence any simplified prediction technique would have to include both these parameters.

The determination of whether or not full bore flow will occur at any particular combination of flow, $Q$, roughness, $n$, and pipe slope, $S_{O}$, is rather simpler, depending on a comparison of normal depth to pipe diameter.

Figure 8 presents the normal depth to pipe diameter ratio as a function of a term nQ/s 172 that may be seen to determine normal depth from the Manning expression, equation (2). It will be seen that the curves for each of the three pipe diameters tend to become linear at the higher values of $h_{n} / D$. Thus a general equation may be proposed to indicate whether full bore flow is achievable, from figure 8,

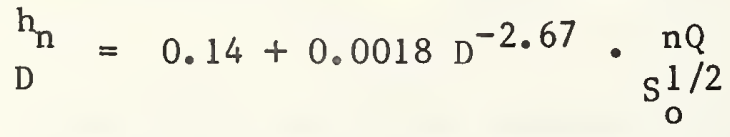

Once the establishment of full bore flow has been predicted it becomes necessary to determine whether this will occur. within the system pipe length. The tables presented in Appendix 1 allow this calculation provided that the inflow rate and specific energy are known together with pipe slope, roughness and diameter.

Obviously if the transition length predicted is less than the required pipe length then design alternatives would include an increase in pipe slope, an increase in pipe diameter or the use of a smoother pipe, although it is recognized that this last alternative is unlikely to be employed.

The use of such tabular data is considered practical, however a graphical technique would have advantages in terms of the appreciation of effect of rapid design changes.

The problem to be solved here is the dependence of transition length on entry energy, however this may be bypassed if the entry depth is calculated.

Figure 9 presents the entry depth for any flow rate and specific energy combination for the three pipe diameters condensed in the form

$$
\begin{aligned}
& h_{e} / D=f(Q, E) \\
& \left(h_{e} / D\right) / Q=f_{1}(E)
\end{aligned}
$$

Thus for any $Q$ and $E$ values the entry depth $h_{e}$ may be read off figure 9 . 
It was found that the transition length at any pipe roughness, gradient, inflow rate and specific energy could be expressed in terms of

$$
L / D=f\left(h_{n} / D-h_{e} / D\right)
$$

as illustrated in figure 10 .

For example, for a given flow rate the entry depth will decrease as the entry specific energy increases. Hence the depth change to be achieved to either full bore flow or normal depth increases as the entry energy rises. Therefore it would be reasonable to expect that the transition length would increase as the entry depth decreased, as shown in figure 10.

The curves in figure 10 apply to the specified one pipe diameter at one pipe slope at a range of pipe roughness coefficients. Obviously such curves may be generated from the data presented in appendix 1 for all the test cases.

It will be noted that individual points are plotted in figure 10 in the manner normal for test results and display a degree of scatter. This is due to the use of either pipe diameter or normal depth as the depth downstream target, whichever is the smaller value. This leads to a slight incompatibility between transition lengths to either $D$ or $h_{n}$. However this scatter of the order of 2 to 3 percent and is well within the other uncertainties in the design of drainage systems, so that this technique may be proposed as a rapid visual method of determining the effect of design changes.

The tabular data presented in appendix 1 may be utilized if the entry flow rate and specific energy are known. Referring to table 1, reproduced from appen dix 1 , it will be seen that it will be necessary to employ an interpolation technique to determine the transition length if the flow entry rate and specific energy do not match the values given. The curves based on the tabular data presented above indicate that a simple linear interpolation would be sufficient. The format of the data, table l, could be simplified as the approach pipe slope is, as mentioned, only included as a convenient method of determining a range in inflow specific energies.

Similarly the data could be rearranged in terms of constant inflow rate blocks instead of the constant approach pipe slope utilized in appendix 1 and table 1.

The data presented has been based on inflow rate and specific energy and does not include an entry loss coefficient. In practice the form of the entry junction from, for example, a vertical stack to the building drain would lead to a junction loss coefficient that would appear, for any given flow rate, as a reduced specific energy.

Although this loss coefficient is not included in the presented data, it is automatically accounted for in the prediction method outlined above. A reduction in specific energy for any flow rate effectively increases the entry depth, figure 9, and hence reduces the transition length, figure 10, for any one set of pipe design parameters. 
In the absence of published data on such loss coefficients, experimental work is suggested to fill this gap and the inclusion of an estimated loss coefficient is recommended in the application of the presented data. 


\section{CONCLUSIONS}

In supercritical flow in a partially filled pipe a transition region may be identified at the pipe entry that allows the flow depth to increase to that compatible with the flow rate and pipe parameters (slope, roughness, diameter, etc.).

This transition may lead to the establishment of full bore flow if the pipe exceeds the necessary transition length at flow rates where the normal depth, as predicted by Mannings equation, exceeds the pipe diameter.

This study has identified the conditions necessary for full bore flow and has determined the transition lengths at a range of flow rates and pipe design parameters compatible with current drainage design.

It is stressed that the design data presented is based on entry flow and specific energy. Although no entry loss coefficient has been included, this effect is automatically accounted for in the design curves presented as an entry loss would merely reduce the flow specific energy at entry and would not affect the validity of predictions based on the data presented. 
6. REFERENCES

1. Swaffield, J. A., Prediction of the Hydraulic Jump Location Following a Change of Slope in Partially Filled Pipe Flow, NBS Report, August 1980.

2. Swaffield, J. A., Application of the Method of Characteristics to Predict Attenuation in Unsteady Partially Filled Pipe Flow, NBS Report, October 1980.

3. Jaeger, C., Engineering. Fluid Mechanics, Blackie and Sons, London, 1956.

4. Chow, V. T., Open Channel Hydraulics, McGraw Hill, 1970. 

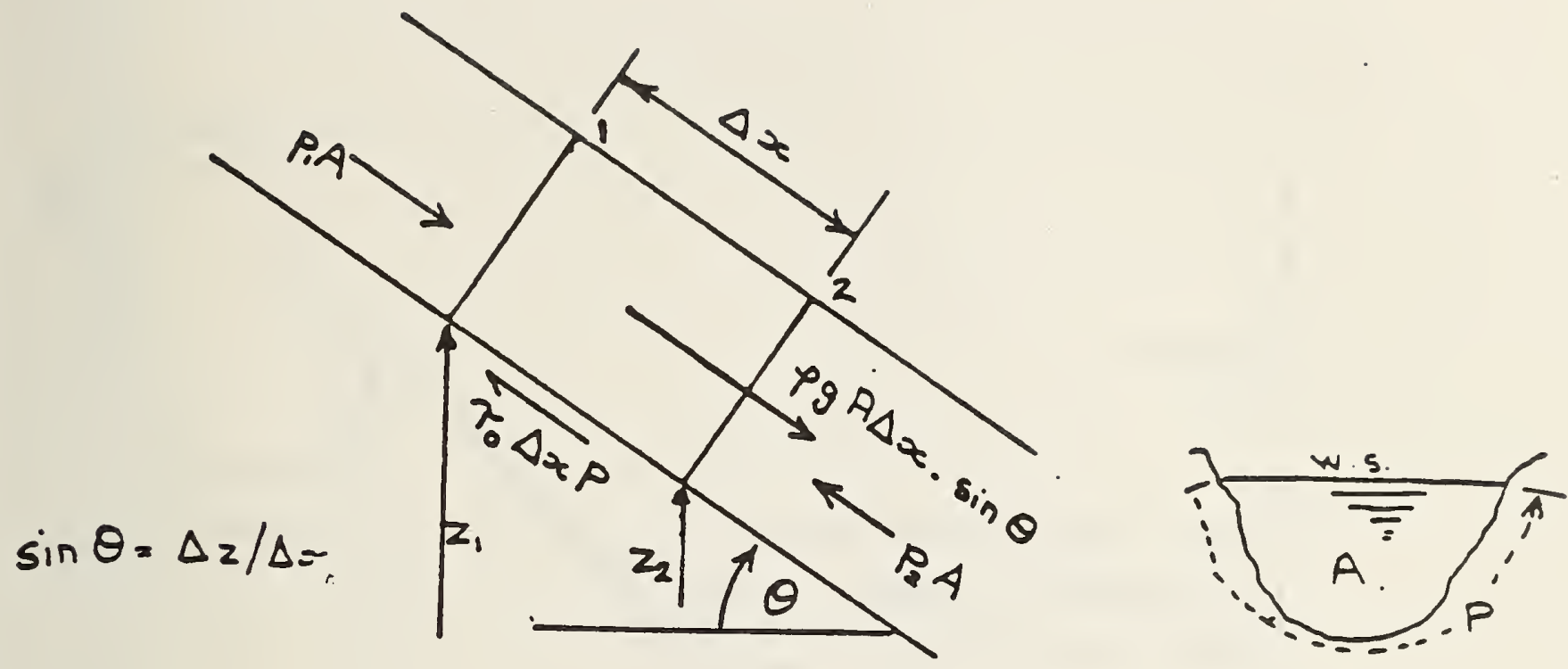

From energy equation $1-2$

$$
\text { Losses }=h_{f}=\frac{p_{1}-p_{2}}{p g}+z_{1}-z_{2}
$$

as $V_{1}=V_{2}$; steady, uniform flow.

From momentum equation down slope

$$
\left(p_{1}-p_{2}\right) A+\rho g A \Delta x \sin \theta-\tau_{0} \Delta x P=0
$$

as $d V / d t=0$; study flow.

$$
\therefore \quad \frac{p_{1}-p_{2}}{\rho g}+\Delta z=\tau_{0} \frac{\Delta x p}{\rho g A}=h_{f}
$$

For turbulent flow $\tau_{0}=f \frac{1}{2} p v^{2}$

$$
\begin{aligned}
h_{f}=f \frac{\Delta x v^{2}}{2 g m}, v=C \sqrt{m S_{0}}, & S_{0}=\sin \theta \\
m & =A / P \\
C & =\text { constant }
\end{aligned}
$$

Figure 1. Derivation of Chez's equation for free surface flow

15 

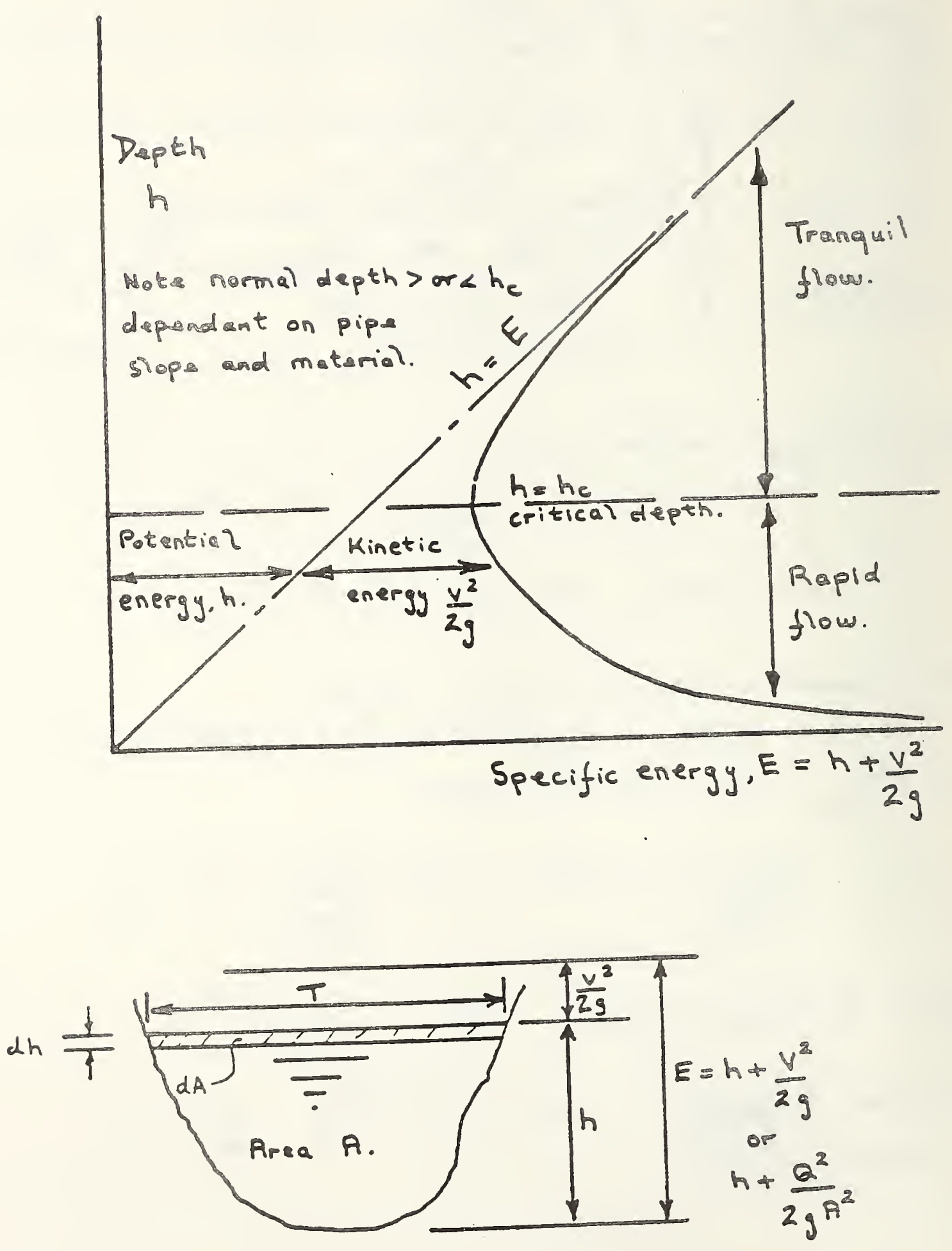

Figure 2. Relationship between flow specific energy and flow depth 


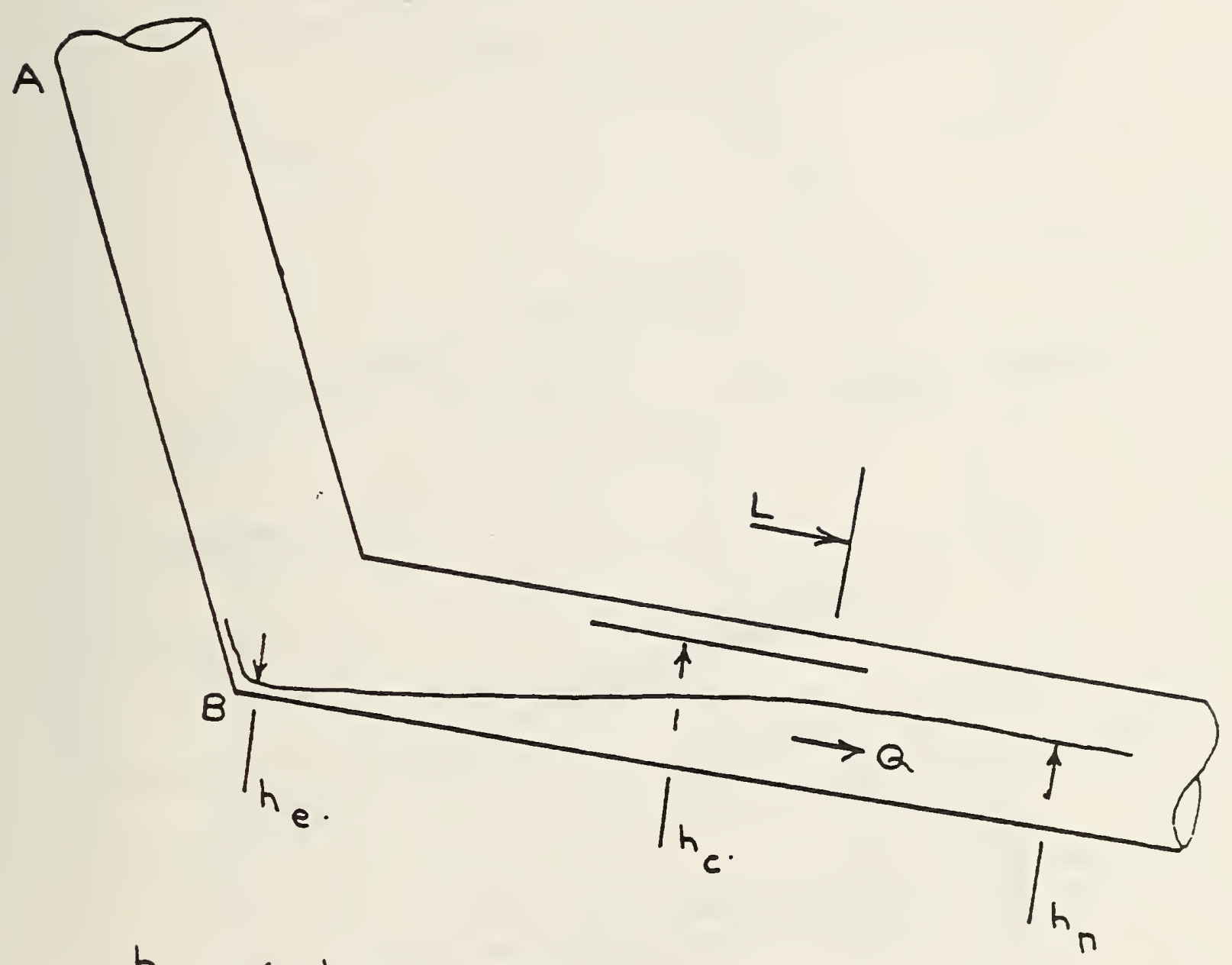

$h_{n}<h_{c}$, flow supercritical, no hydraulic jump forms.

$$
h_{e}=f(Q, E), \quad E=h_{e}+V_{e}^{2} / 2 g
$$

Transition length $L=f\left(h_{e}, h_{n}\right)$

Note transition length taken as $h$ value for $h=0.975 h_{n}$.

Figure 3. Transition length downstream of a pipe slope change in partially filled pipe flow

17 


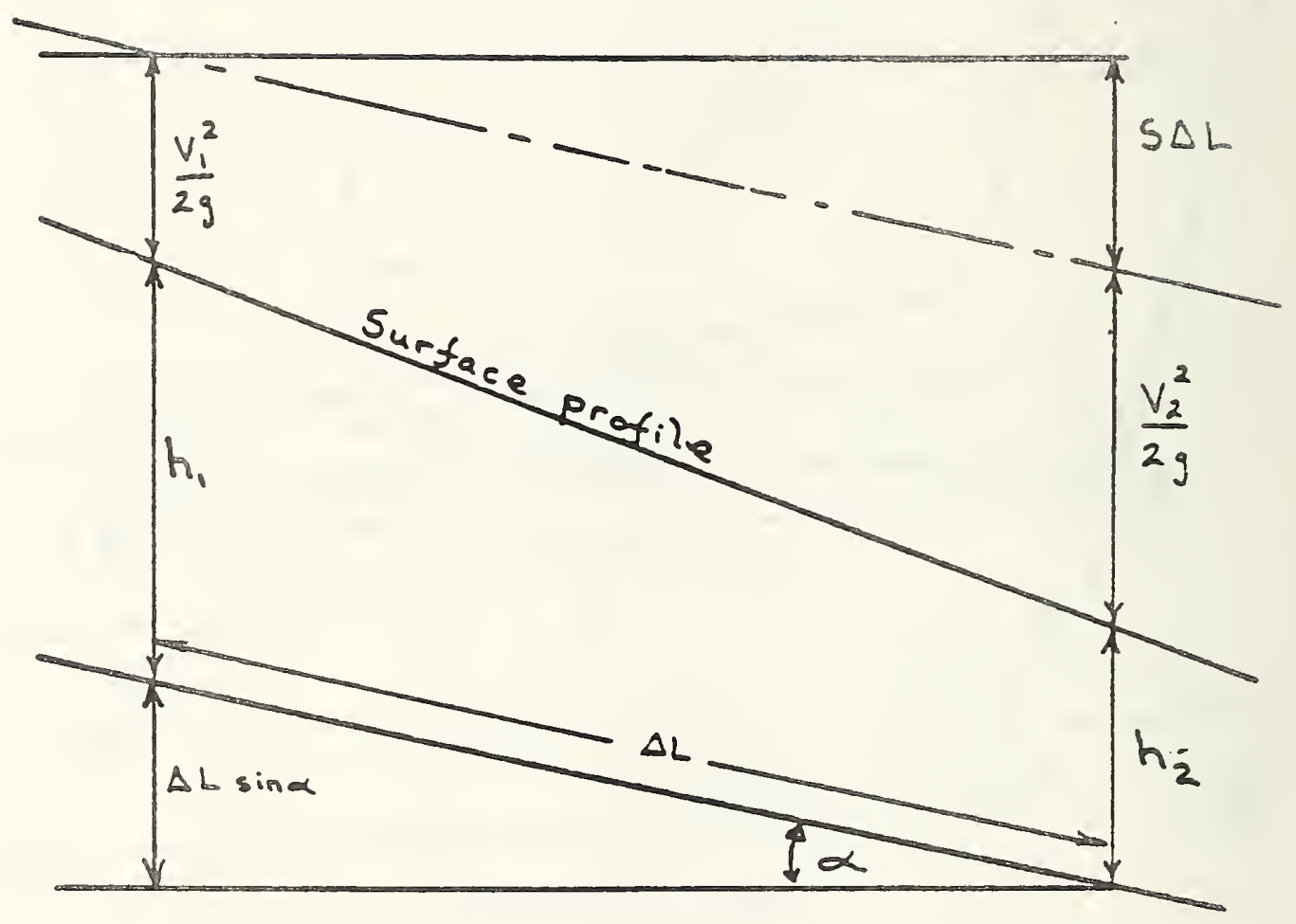

Grodually varied flow, analysis based on heed loss at any sectien being equel to manning loss prediction, where

$$
S=-\frac{\Delta E}{\Delta L}=\left(\frac{n Q}{A m^{2 / 3}}\right)^{2}
$$

Figure 4. Basis of gradually varied flow analysis 
Transition zone

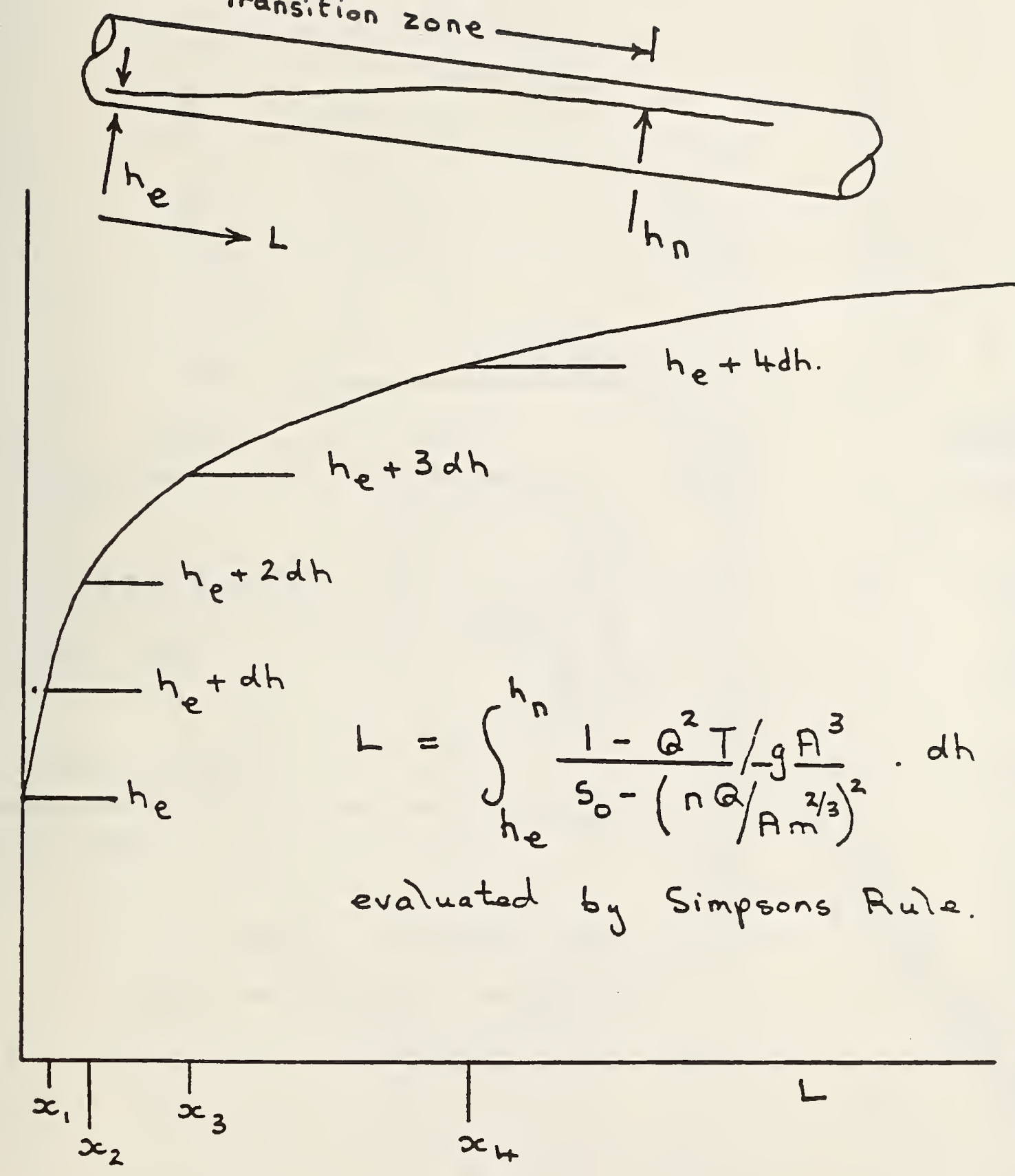

Note (i) $\Delta L$ increases as $L$ increases

(ii) water surface profile approaches normal depth as shown only in supercritical flow

Figure 5. Schematic representation of numerical integration to determine water surface profile

19 


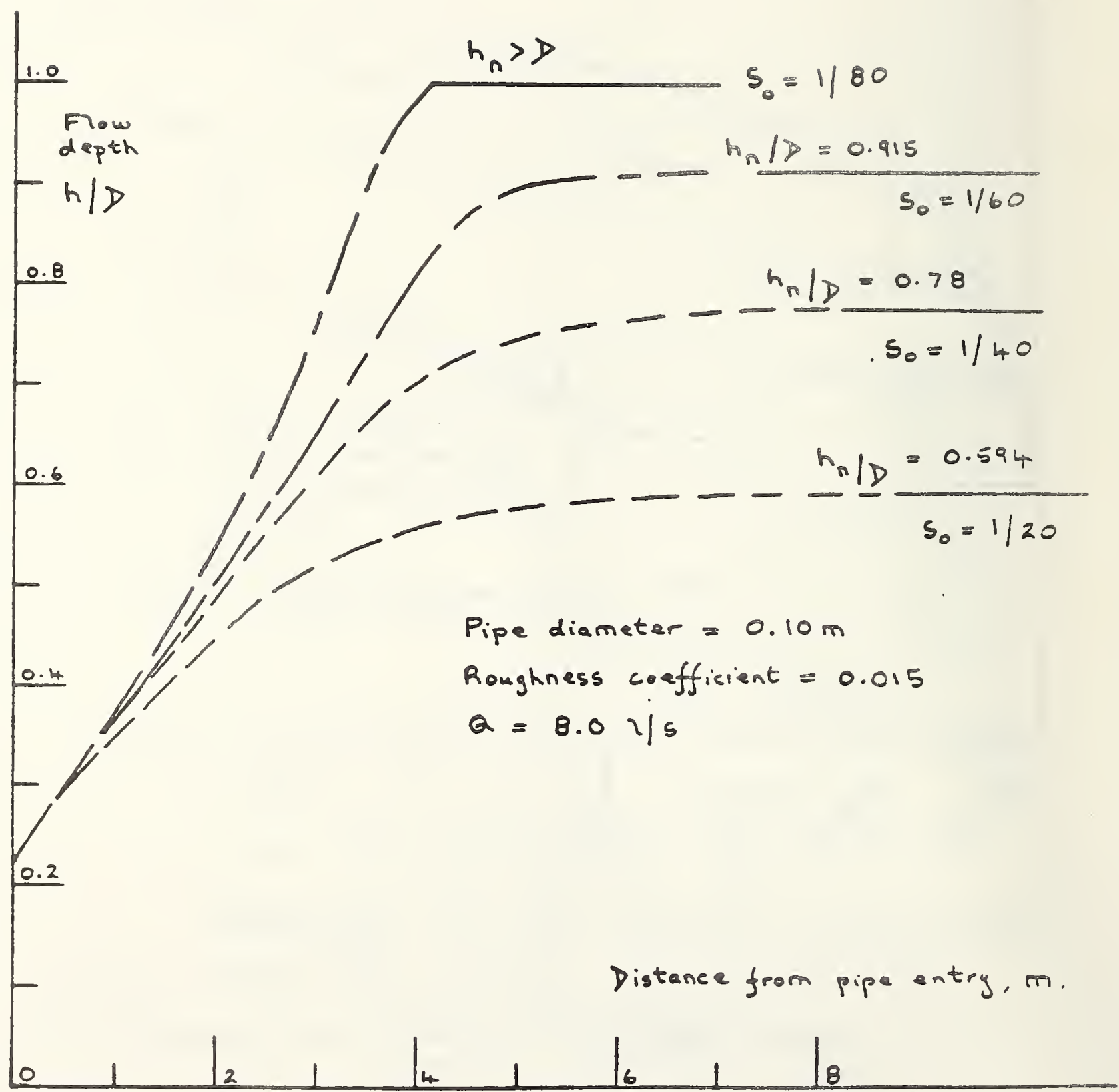

Figure 6. Water surface profiles for a range of pipe gradients, illustrating increase in transition length as pipe slope is increased 


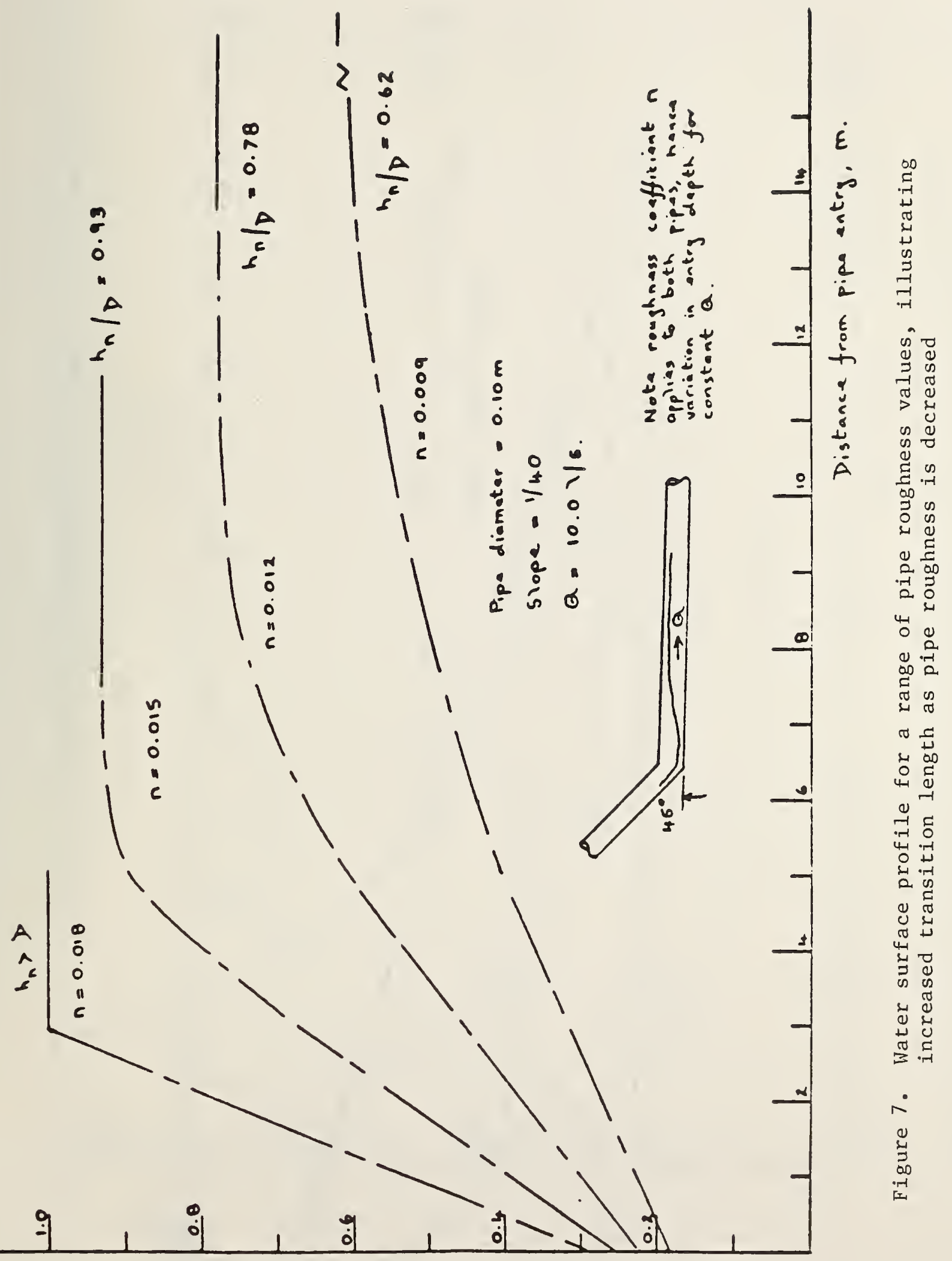




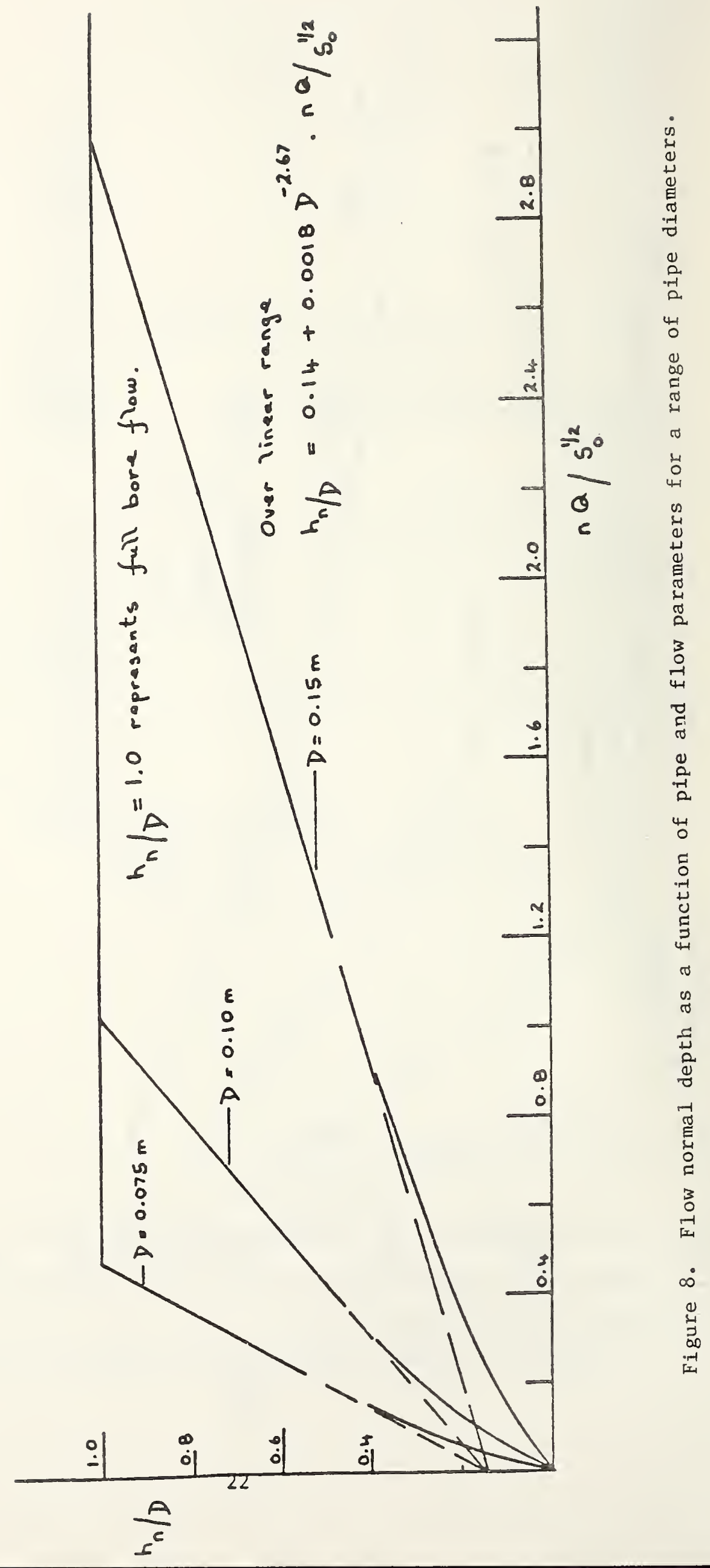




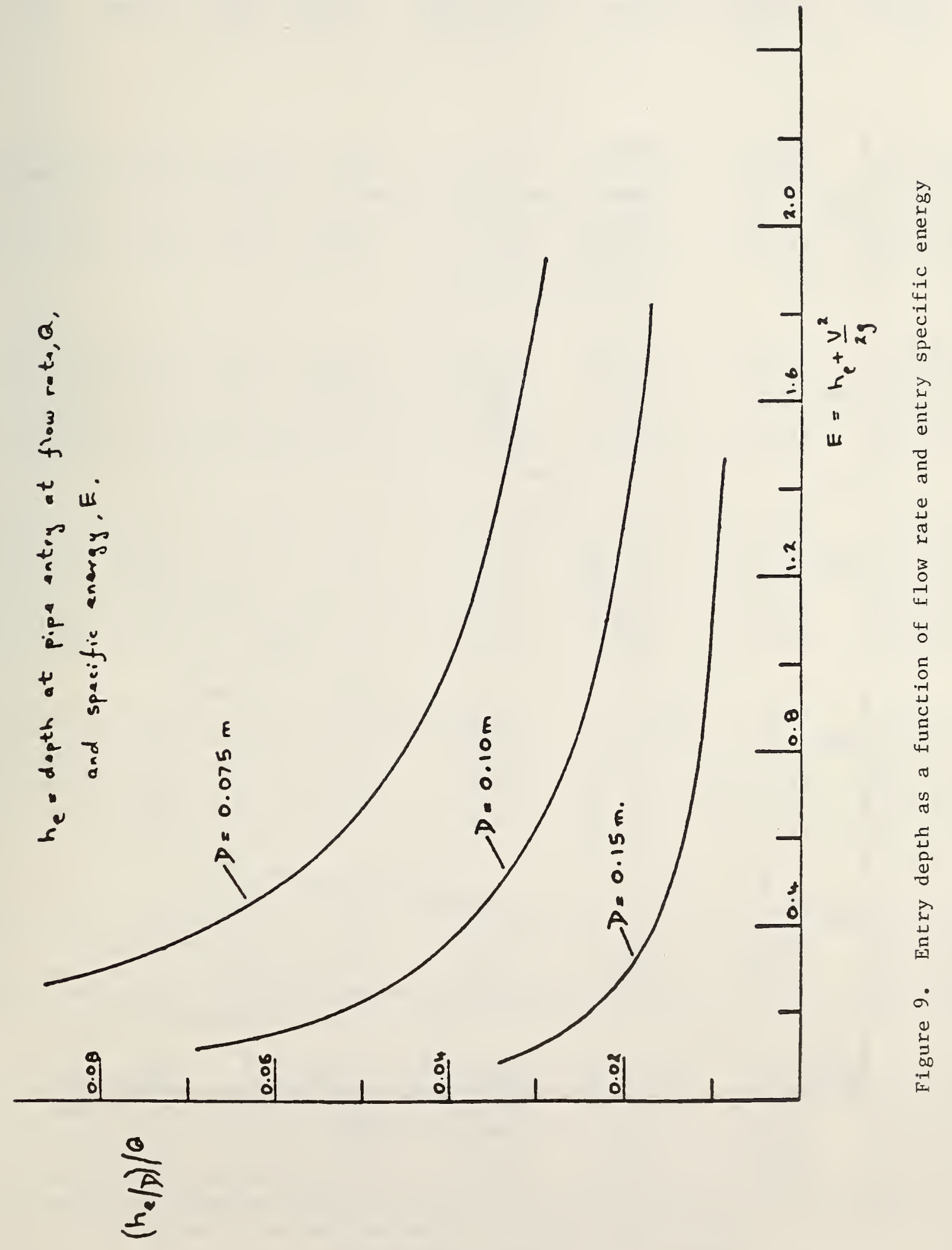




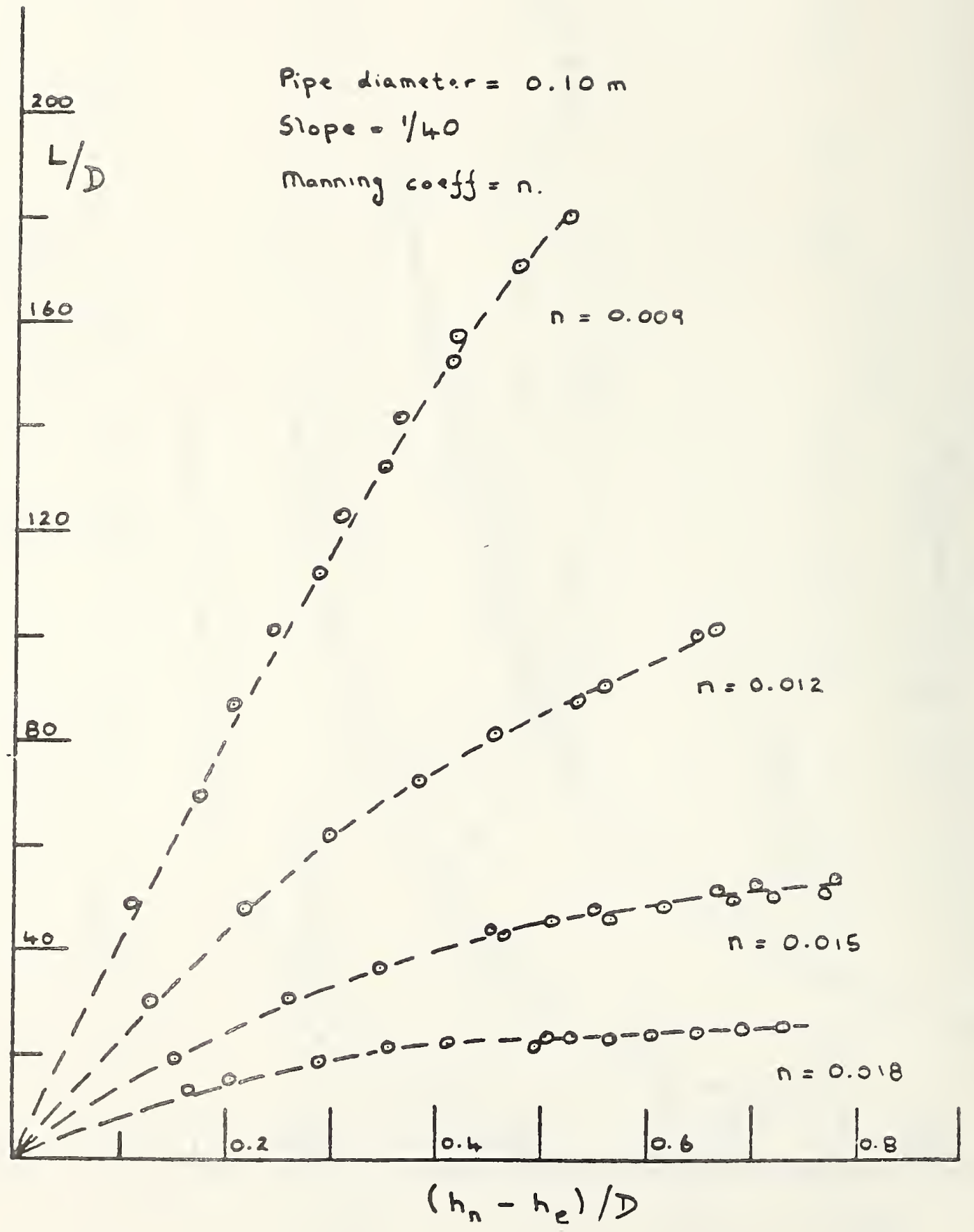

Figure 10. Transition length as a function of depth change from entry to normal depth, note that flow rate and entry energy are subsumed in the depth change term 


FLOH DIA. MAYH.
LIS. H. COSFF

SUPPLY CFAIN

SLCPE SLCPE

(SIN) ISIN)
ORAIN FLOH

ENTRY DEPTH

RATIO H/O. .
ENTRY

ENERGY

n.
NORMAL DEPTH

H/D.
PIPL LEHETH TC NORMAL JEPTH. L/U.

\begin{tabular}{|c|c|c|c|c|}
\hline a & 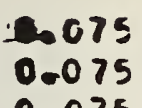 & 0 & 0 & 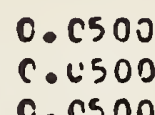 \\
\hline & & $\begin{array}{l}0.00>0 \\
0.00>0 \\
0.0 .0>0\end{array}$ & $\begin{array}{l}0 \\
0 .\end{array}$ & $\begin{array}{l}10 \\
00 \\
\text { no }\end{array}$ \\
\hline
\end{tabular}

$2.000 . C 750.00700 .3 C C C$ C. 0.530 $4.000 .0750 .37700 .5 c c 0 c .0500$ 6.00 C.C75 C.0740 $0.5 \operatorname{ccc} C . C 500$

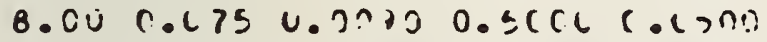

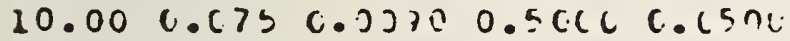

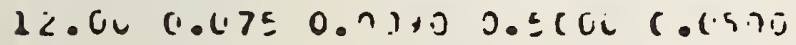

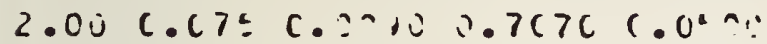
4.00 C..L7E C. $30700.7 C 7 C$ C.C.5 .J $6.00 \quad 0.1750 .10700 .7 C 7$ C. $\quad(590)$

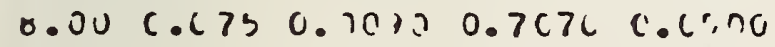

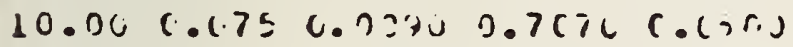

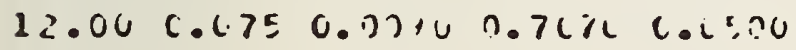

\begin{abstract}
0.1493
0.2384

0.3167

C. 3594

0.4507

c. $52=9$
\end{abstract}

0.1204

c. 19006

$0.25 i 2$

c. 3.374

c. 3600

c.4124

(. 1070

U.164t

0.2230

$4.27: 2$

0.3230

U. 3032

0.1208

$0.150 \mathrm{C}$

U. 20,1

c. 2537

U. 2400

4.3372

0.0974

0.1530

c. 20 ve

U. 2444

ن. 2354

3.3245
0.3003

0.4714

0.0021

0.7491

0.7449

0.0782

0.4534

0.7241

0.9377

1.1143

1.0059

$1.4(11)$
0.2605

0.4250

0.5746

0.7241

0.0447

1. 0000
53.3110

90.4052

112.4573

130.7793

154.0711

170.5312

$0.2 t: 05$

0.4200

$0.37 \times 6$

0.7241

U. 9047

1. กi)

0.2640

0.7092

1. $182 \mathrm{~h}$

1.4117

1.0105

1.7572

j. 20.15

U. $42=0$

$0.57 \times 6$

$0.7<41$

0.8497

1. is Jus

01.7107

1 บU.57บ?

124.5074

129.0747

$173.24=0$

$210.71,71$

04.2311

1しン. $2 \vdots 4<$

1:2. 3713

159.754 4

1.1. 1.1:7

(2).972?

0.2 tis

$0.42=0$

$0.57 \times 0$

0.7241

v. Ond?

1.000u

0.2305

$0.42=0$

J.5740

U. 7241

0.8647

1.0000

65. SL25

$107.12=7$

i $37 .+4=2$

12. .225

$\therefore=: 0<10$

23<.

Oc.15j=

$100.17+7$

134.3301

$104.13=3$

1E $7.27=0$

<.o.t.1., 7

L.e.3.3!4

$0.0904 \quad 0.7013$

0.26 .5

100.4 143

U. $1>12 \quad 1.1961$

$0 .+2.0$

$134.74,2$

$1.4=57$

$0.57 \times 0$

U. $72+1$

$10 \% .4 i, 1$

U. 2410

0.5347

1 $5 \%$. his

23i.33)

Table 1. Typical Tabular Data for $0.10 \mathrm{~m}$ Diameter Pipe 
APPENDIX 1

TABULAR DATA RELATING FLOW NORMAL DEPTH AND TRANSITION LENGTH TO FLOW AND PIPE PARAIETERS

$$
\begin{aligned}
& \text { Cases included: } \\
& \begin{array}{l}
\mathrm{D}=0.075 \mathrm{~m}, \mathrm{Q}=2-12 \mathrm{l} / \mathrm{s}, \mathrm{n}=0.009-0.018 \\
\mathrm{D}=0.10 \mathrm{~m}, \mathrm{Q}=2-12 \mathrm{l} / \mathrm{s}, \mathrm{n}=0.009-0.018 \\
\mathrm{D}=0.15 \mathrm{~m}, \mathrm{Q}=2-12 \mathrm{l} / \mathrm{s}, \mathrm{n}=0.009-0.018 \\
\mathrm{D}=0.15 \mathrm{~m}, \mathrm{Q}=14-22 \mathrm{l} / \mathrm{s}, \mathrm{n}=0.009-0.018
\end{array}
\end{aligned}
$$




$$
D=0.075
$$




FLOH DIA. MAYY. SUPPLY CKAIM
L/S. H. COSFF SLCPE SLOPE

$2.00 \quad 0.0750 .72900 .25060 .0500$

$4.00 \quad 0.075 \quad 0.7270 \quad 0.25+t \quad 6.4500$

6.00 0.Cis c. on?0 0.żEt 0.esno

8.00 C.C75 0.29100 .8500 C.c1500

$10.00 \mathrm{C} C .675$ i. $30700.25 t e 0 .(50)$

$12.00 \quad 0.0750 .00100 .256 t \quad .05 n 0$

$2.00 \quad 0.0750 .00300 .5060 \quad 0.0500$

$4.00 \quad 0.075 \quad c .37300 .5$ CCC 0.0500

$6.00 \quad 6.075 \quad$ G.07.0 0.5CCC C. 0500

$8.00 \quad 0.675$ U. $30700.5 C C L C .1500$

$10.00 \quad 0.075 \quad 0.3370 \quad 0.5000$ C.C500

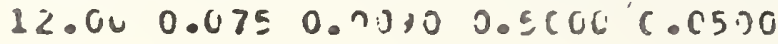

ORAIN FLOH

ENTKY DEPTH

RAIIO HIO.
ENTRY

ENERGY

ก.
MORMAL

DEPTH

HOD.
PIPE LEISTIH TI NORMAL JEPTH.

L/U.

\subsection{3 \\ 0.2344 \\ 0.3167 \\ $\because .3394$ \\ 0.4507 \\ 30.5234}

0.3003

0.4714

0.6021

0.7692

0.7499

0.0782

0.4534

0.7241

0.9377

1.1143

$1 .<659$

1.4015

0.1076

0.1696

0.2230

4.2722

u. $320 t$

U. 3630
0.5646

0.4092

1.1826

1.4117

1.0103

1.7872
0.2605

0.4230

0.5746

0.7241

0.8647

1.00ن
53.3110

90.4602

115.9573

130.7753

154.6711

170.3312

$0.26 \cup 5$

0.4200

0.5746

0.7241

0.5647

1.0000

0.2605

0.4200

0.5746

U. 7241

0.8547

1.0000

0.0422

.1 .0369

1. 3543

1.0213

i. 0530

2.0659

61.7167

100.5702

124.5654

153.0547

$173.24<5$

210.7971

105.0192

135.0715

159.7544

161.0117

225.0757

65.5230

207.1253

$137.94=2$

103.2259

$109.4<70$

$23<$. प्र००
0.2605

0.4230

$0.57 \times 6$

0.7241

U. 3647

1.0000
64.2397

0.2505

$0.42=0$

3.5740

0.7241

0.4647

1.0000

2.2277

0.7010

1.1381

1.4087

1.7047.

2.0470

2.2740
0.2605

0.4200

0.5740

4.8047

1.0000
0.7241
60.1532

100.1747

139.3343

$104.1 \% 23$

187.2720

230.61 .27

60.3314 100.4443 134.7435 16\%.474 J $167.31,3$ 237.532. c. 3210 
FLOH DIA. MANN. SUFPLY OPAIN
LIS. M. COFFF SLCPE SLUPE
ISINI ISINI
DRAIN FLOH ENTRY DEPTH RATIO MIO.
ENTRY

ENERGY

M.
NORMAL OEPTH

H/O.
PIPE LEIAGTH TO NORMAL DEPTH. L/O.

\begin{abstract}
$2.00 \quad 0.0750 .0990 \quad 0.25820 .0250$
$\begin{array}{llllll}4.00 & 0.075 & 0.1070 & 0.2586 & 0.0 .250\end{array}$

$\begin{array}{lllllll}6.00 & 0.075 & 0.0070 & 0.2586 & 0.0250\end{array}$

$8.00 \quad 0 . c 1750.3710 \quad 0.268 .6 \quad 0 .(1 \geq 50$

$\begin{array}{llllll}10.00 & 0.075 & 0.2730 & 0.25 \varepsilon \varepsilon & 0.0253\end{array}$

$12.00 \quad 0.075 \quad 0.0070 \quad 0.2584 \quad 0.0250$
\end{abstract}

\begin{abstract}
0.1493
0.2384

0.3167

0.3244

0.4567

10.5259
\end{abstract}

0.1204

0.1900

c. 2512

0.31174

0.3606

0.4114

0.1076

0.1696

0.2230

0.2722

$0.31=6$

0.3530

0.1008

0.1506

0.2001

c. 2537

0.2766

0.3372

0.0974

0.1530

c. 2046

0.2444

0.2354

0.3245

0.0964

0.1512

0.1994

0.2410

$0.2+\angle 0$

0.3210
0.3003

0.4714

0.6021

0.7091

0.7994

0.0732

0.4534

0.7241

0.4377

1.1143

1.2659

1.4015

0.5646

0.9092

1.1023

1.4117

1.0108

1.7872

0.0421

1.0369

1.3543

1.0213

1.0530

2.0659

\subsection{2}

1.1131

1.4537

1.7445

1.9990

2.2277

0.7010

1.1381

1.4887

1.7047

2.1479

2.2746
0.3328

0.5542

0.7593

0.9536

1.0000

1. 0000 .

0.3328

0.5542

$0.75 \times 3$

0.9556

1.0000

1.0000

0.3328

0.5542

$0.75+3$

0.9536

1.0000

1.0000

0.3328

0.5542

$0.75 \div 3$

0.9556

1.0000

1.0000

0.3328

0.3542

0.7593

0.9556

$1.00 v 0$

1.0000

0.3328

0.5542

$0.75>3$

0.9536

1. 0000

1.0000
74.7487

110.5603

147.3901

160.7624

100.4435

82.4533

80.1539

123.5271

159.2294

163.1312

122.8343

101.4159

82.4540

124.3949

164.3577

$184.4 \angle 02$

133.7221

$110.32>0$

83.6575

131.41330

$107.07 \leq 1$

192.7649

137.8971

115.4011

84.2522

132.4250

160.4523

194.4705

140.0367

117.7731

84.4212

132.7300

160.8524

$1 \times 4.91,22$

$14 \mathrm{~L} .71 .2$

$11 c .4 i=0$ 
FLOM DIA. MANN. SUPPLY CRAIN L/S. H. CJEFF SLEPE SLGPE ISINS ISINI
DRAIN FLCH ENTRY ENTRY OEPTH. ENERGY RAI 10 HJO.
NORMAL DEPTH MD.
PIPE. LEIGTH TI NORHAL DEPTH. LID.

\begin{abstract}
$\begin{array}{llllll}2.00 & 0.075 & 0.0070 & 0.2583 & 0.0167\end{array}$
$4.00 \quad 0.075 \quad c .3970 \quad 0.2560 \quad c .4167$

$6.00 \quad 0.0750 .0090 \quad 0.25 E$ \& $\quad 0.0167$

8.0C C.0.75 $0.32 .30 \quad 0.25 E E$ C.C.257

$10.00 \quad 0.075 \quad 0.3270 \quad 0.25 E 80.0167$

$12.00 \quad 0.0750 .0030 \quad 0.25860 .0157$
\end{abstract}

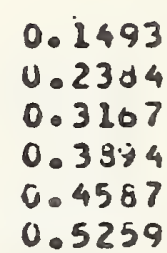

0.1204

0.1906

0.2512

0.3074

0.3606

0.4114
0.3003

0.4714

0.0021

0.7091

0.7999

0.0782

0.4534

0.7241

$0 .+377$

1.2143

1.2659

1.4015

0.5646

0.4092

1.1826

1.4117

1.0108

1.7872
0.1008

0.1536

0.2001

0.2537

0.2960

0.3372

0.0974

0.1530

प. 2040

0.2444

0.2554

0.3245

0.0421

1.0369

1.3543

1.6213

1.0530

2.0659

0.6872

1.123 .1

1.4567

1.7445

1. 4990

2.2277

0.0964

0.1512

0.1934

0.2410

ن. 2520

0.3220
0.3850

0.6470

0.3921

1. 0000

1. U0ua

1.0000

0.3850

0.6470

0.3921

1.0000

1.0000

1.0000

$0.38,0$

0.6470

0.6921

1.0000

1.0000

1.0000

0.3850

0.6470

0.8921

1.0000

1.0000

1.0000

0.3950

0.6470

0.8421

\&. 0000

1.0000

1. 0000

0.3850

0.6470

0.8921

1.0040

1. 0000

1.0000
84.0454

126.7447

15303044

116.0 .9 .57

40.0043

$73.045 \%$

84.2367

135.4402

167.4073

134.7243

100.3604

92.0247

91.4912

139.2714

172.17 .1

230.3745

114.5910

$101.2055^{\circ}$

92.6923

147.0222

170.3521

141.4461

110.7400

105.9259

93.2547

150.7227

170.3251

143.7405

126.5152

$10.0 .27+3$

$93.31 \% 1$ 150.4101 170.1441 144.2433

$121.54: 5$

100.7125 


FLOH DIA. MAIN.
L/S. M. COEFF

SUPPLY DOAIN

SLCPE. SLCPF

(SIN) (SIN)
DKAIN FLOH

EMTRY DEPTM ENERGY

RATIO HID.
ENITRY
ENERGY

M.
NURMAL DEPTH H/D.
PIPE. LEIVGTH TO NOKMAL JEPTH. LSO.

\begin{abstract}
$2.00 \quad 0.075 \quad 0.0070 \quad 0.2588 \quad 0.0125$
$4.00 \quad 0.0750 .13070 \quad 0.2562 \quad 0.4125$

$6.00 \quad 0.0750 .1970 \quad 0.2560 \quad 0.0125$

B.00 $0.075 \quad 0.32: 0 \quad 0.25$.

$10.00 \quad 0.075 \quad 6.02700 .2528 \quad$ C.6123

$12.00 \quad 0.075 \quad 0.01 .100 .25 \varepsilon 0 \quad 0.0125$
\end{abstract}

\begin{abstract}
0.1493
0.2304

0.3167

0.3844

U. $4 \div 37$

0.5259
\end{abstract}

C. 1204

$0.190 t$

0.2512

0.3074

U. 3006

U. 4114

i. 1076

$0.16 \% t$

0.2230

$0.27<2$

0.3106

$0.30 s$ is

0.10 .58

0.1526

0.2031

ن. $25 \$ 7$

0.2760

ن. 3372

C. 0974

0.1510

ن. 23.16

0.2444

c. 2354

U. 324 :

0.0904

c. 1512

0.1904

c. 2410

0.2360

0.3210
0.3003

0.4714

0.0021

0.7041

0.7994

0.0742

0.4534

0.7241

0.4377

1.1143

1.2659

1.4015

0.5340

0.4092

1.2026

1.4117

1.0108

1.7872

0.6421

1. 6369

1.3543

1.0213

1.0530

2.005t

0.0872

1.2131

1.4567

1.7445

1. 4970

2.2277

0.7010

1.1381

$1.4=67$

1.8347

2.3470

$2 .<745$
0.4280

0.7241

1. 0000

1.0000

1.0000

1. 0000

0.4280

0.7241

1.0000

1.0000

1.01000

1.0000

0.4280

0.7241

1.0000

1.0000

1.0000

1. 0000

0.4200

0.7241

1.0000

1.0000

1.0000

1. 0000

0.4200

0.7241

1.0000

1.0000

1.0000

1.0000

0.4200

0.7241

1.0000

$1.00 \cup 0$

1.0 .000

1.01100
87. 0075

120.2355

103.24>7

101. 2007

93.0233

70.0330

93.0496

130.7601

$18 \mathrm{u} \cdot 100 \mathrm{~s}$

110.1131

100.3729

80.7008

95.2693

$144.537 j$

$180.7 \angle 23$

$122.64: 4$

$100 \cdot 3: 5 \% 2$

$97.24 \div 2$

94.1412

144.0031

190.2013

$120 \cdot 23.3$

112.0145

$101 .+730$

$93.57 \times 4$

15<.32

192.0 .043

120.0131

114.2115

104.3304

93.405:

152.11 .7

19c.5:1'3

126.00.0

$114 .=271$

$104.90 ; 1$ 


\begin{abstract}
FLOH DIA.
tis. n.

MANK.

COEFF
\end{abstract}

DRAIN FLOH

ENTRY DEPTH

RATIO H/O.
EMTRY

ENERGY

He
MORHAL

DEPTH

$\therefore$.HOO
PIPE LENGTA 1

MORAAL UEPTH. L/D.

:

36.6329

57.1439

72.1624

84.3317

56.6411

37.2790

40.7864

63.8034

80.7602

94.5010

60.5540

50.2442

\subsection{6 \\ 0.2059 \\ 0.2722 \\ 0.3333 \\ 0.3918 \\ 0.4475}

0.1214

0.1723

0.2537

0.3103

0.3640

0.4153

0.1172

0.1852

0.2444

C. 2450

$0.34+9$

U. 3492

0.1160

0.1832

0.2410

0.2952

0.3434

0.3945
0.39 .24

0.0232

0.0030

0.7533

1.0785

1. 2918

0.4463

0.7117

0.9203

1. 4940

1.2430

1.3762

0.4775

0.7655

0.4693

1. 1788

1. .4423

1. 4057

0.4373

0.7014

1.0116

1.2055

1.3719

1.5180
0.3191

0.5248

0.7241

0.9116

1.0000

1.0000

0.3191

0.5298

0.7241

0.9116

1. 0000

1. 0000

0.3141

0.5248

0.7241

0.9110

1.0000

1.0000

0.3141

0.5248

0.7241

0.9116

1.0000

1.0000
42.4726

$60.55 i .3$

84.4010

90.9052

$330: 00004$

56.1702

43. 3450

67.9977

86.3142

101.1972

70.7545

54.3613

43.7702

60.7412

87.2702

142.3732

70.2510

60.9831

43.9010

60.4431

37. 5030

102.7105

70.6030

$61 \cdot 4:=3$ 


FLOH OIA. MANV.
L/S. H. CIJEFF

SUFPLY TRLI:

SLUPE SLCPE (SIN) (SIIA)
DRAIN FLOH

ENTKY DEPTH

RAIIT M/C.
ENTRY

ENERGY

n.
NORMAL UEPTH

H/O.
PIPE LEYGTH TC MORMAL UEPTH. L/D.

\begin{abstract}
$2.00 \quad 0.075 \quad 0.0120 \quad 0.25480 .4250$
4.00 C.C 75 $0.31200 .2660 \quad 0 .(1250$

$6.00 \quad 0.075 \quad 0.312 C \quad 0.2560 \quad C .(250$

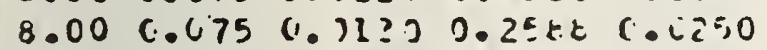

$10.00 \quad 0.0 .75 \quad 0.171200 .2586 \quad 0 .(250$

$12.00 \quad 0.075 \quad 0.21200 .2380 \quad 0.4250$
\end{abstract}

\author{
0.1810 \\ 0.2413 \\ U. 3344 \\ 0.4312 \\ U. 5640 \\ ن. 6548
}

0.1454

0.2316

U. 3074

0.3777

6.4446

C. 5093

0.1290

0.2059

0.2722

4.3313

v. 3:316

U. 447 E

0.1214

0.1923

0.2537

i. 31 ن 3

C. $364 \mathrm{C}$

0.4153

0.1172

0.1322

U. 2444

c. 29.96

$\therefore 3449$

i. $314 \mathrm{c}$

(1. 1100

v. 1332

0. 2430

$0.27,2$

U. 34.519

u. $3+44=$
0.2102

0.3257

0.4126

0.4013

0.5359

0.3901

0.3157

0.4480

0.0369

0.7511

0.0453

0.4325

0.3924

0.0232

0 -díl

0.4533

1.47 . 5

1.1916

0.4463

0.7117

0.4203

1. نप4

1. (43)

1.3762

0.4775

0.7655

$0.1=93$

1.17 is

1. 3423

1.4051

0.4573

0.78 .14

1. 4110

1.<us?

1.371 .

$1.51<0$
0.4049

0.6919

0.4550

i. 0000

1.0000

1.0000

0.4049

0.0919

0.9556

1. 0 DL 0

1. on 00

1.0000

$0.40>9$

0.6719

0.9356

1.0000

1.0000

$1.00 \cup 0$

J. 4099

$0.5+14$

$0.93>b$

1. .0000

1.0000

1.0000

0.4097

0.6914

$0.95>0$

1. viruo

1. 1000

1. 00,0

0.40 .84

0.t919

$0.95>0$

1. 1) U⿺辶 0

1. 0 1.

1. 00u
$45.57<4$

67.2437

80.0209

51.4024

$35.05=3$

29.5558

$49 \cdot 16+4$

73.1474

$87.77<1$

60.9 .355

49.5017

41.5505

50.7431

72.7575

$91 \cdot 2 \cdot 14$

$65.45: 7$

34.5434

$47.23<5$

51.5213

$77.10 \leq 7$

93.1075

$07.73+7$

37.41.3

$50.350 y$

51.0901

77.5431

94.1404

04. ?2.2

$3=041=$

$5+1112$

50.1670

7c. נ4:

$94 \cdot 4 c>2$

6). 37.2

5i. 2911

32. 3443 
FLDH DIA. MANN. SUPFLY CFAIN L/S. :H. COEFF SLOPE SLLPE (SIN) (SIN)
DRAIN FLOW ENTRY ENTRY OEPTH ENERGY RATIO MID.
MURMAL DEPTH

n.
HOO.
PIPE LEMGTH TO MORMAL JEPTHO $L / D$.

\section{$2.00 \quad 0.0750 .0120 \quad 0.25680 .0267$ \\ $4.00 \quad 0.075 \quad 0.3120 \quad 0.2565 \quad 0.0167$ \\ $6.00 \quad 0.075 \quad 0.21 \geq 0 \quad 0.2560 \quad 0.0167$ \\ 8.00 $0.0750 .01200 .2540 \quad 0.0157$ \\ 10.04 G. $(.750 .31: 00.25$. 0.0 .0137 \\ $\begin{array}{lllllll}12.00 & 0.075 & 0.0120 & 0.2546 & 0.0167\end{array}$}

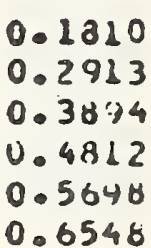

0.1454

0.2316

0.3074

0.3777

0.4446

U. 5073

\subsection{2}

0.3257

0.4116

0.4813

0.5384

0.5901

0.3157

0.4780

0.0369

0.7512

0.0483

0.9326

0.3924

0.0232

0.2030

0.4533

1.0785

1.1918
0.4753

0.8110

1.0000

1.0000

1.0000

1.0000

0.4753

0.3110

1. 0000

1.0000

$1.00 \cup 0$

1.0000

0.4753

0.8110

1.0040

1.0000

$1.00 \cup 0$

1.0000
46.1400

63.7326

50.0000

44.4526

35.4055

27.7305

49.6925

69.61 .16

64.7537

53.0033

4 七. 0744

34.4050

51.2317

72.2713

60.3547

50.1770

51.0614

45.0710

0.4753

0.8110

1.0000

2. 0000

1. 0000

1. 0000

$1.37 t 2$

0.4753

0.8110

1.0000

1.0000

1. 0000

1.0000

52.1350

$75.5,36$

$70.312 ?$

60.5127

53.561 .5

45.1232

53.2285

77.3009

71.3 .504

01.7240

$50.21>0$

44.0531

53.5474

$77 .=425$

71.30120

6i.joull

59.011U

5u. 11 . 5 

FLOH DIA. MANH.
LIS. H. CUEFF
SUPPLY CRAIN

SLOPE SLCPE

(SIN) (SIN)
DRAIN FLCM EMTKY LEPTH RATIO HID.
EATRY

ENERGY

M.
NURMAL DEPTH

H/D.
PIPE LEYGTH TC MORMAL JEPTH. L/D. $\begin{array}{rllll}2.00 & 0.075 & 0.0120 & 0.2588 & 0.0125 \\ 4.00 & 0.075 & 0.1120 & 0.2588 & 0.0125 \\ 6.00 & 0.075 & 0.0120 & 0.2568 & 0.0125 \\ 7.00 & 0.075 & 0.1120 & 0.2586 & 0.1125 \\ 10.00 & 0.075 & 0.0120 & 0.2586 & 0.0125 \\ 12.00 & 0.0 .75 & 0.0120 & 0.2566 & 0.0125\end{array}$

2.000 .6750 .01200 .56000 .0125$.

$\begin{array}{llllll}4.00 & 0.075 & 0.0120 & 0.5000 & 0.0125\end{array}$

6.00 C.C7S 0.02200 .5 CCC 0.4225

$0.00 \quad 0.0750 .31200 .5 C C U \quad 0 .(125$

$10.00 \quad 0.0750 .32200 .3600, c .0125$

$12.000 .0750 .11200 .5 \operatorname{coc} C .01120$

$2.00 \quad 0.0750 .0120 \quad 0.7 C 7 C \quad 0.0125$

$4.00 \quad 0.0 .750 .01200 .70 .76 \quad c .4125$

$6.00 \quad 0.0750 .01200 .7 C 70$ r.0.12?

8.00 C.L.7E $0.01200 .7 C 70$ C.L.125

$\begin{array}{lllll}10.00 & 0.075 & 0.3120 & 0.70 .76 & 0.0125\end{array}$

$12.00 \quad 0.0750 .01200 .7070 \quad 0.0225$

$2.00 \quad C .075 \quad 0.0120 \quad 0 . E \in E$ C.CZ2.5

$4.00 \quad \because .0750 .01 ? 30 . \Sigma$ Ut: C.C125

6.00 C.u $750.31200 . t \in t u 0.0125$

B.00 $0.475 \quad 0.01 \geq 2 \quad 0.5 t$. C. C.2125

$10.00 \quad 0.0750 .31200 .86 t 0 \quad$ C.C.C125

$12.00 \quad 0.6 .75 \quad 0.0120 \quad 0$. ettc C.il?s

$2.00 \quad 0.0750 .5120 \quad 0.9654 \quad 0.0125$

$4.00 \quad 0.0750 .01200 .4654$ C.L125

$6.00 \quad 0.075 \quad 0.01200 .4$ CEY C.0125

E.00 C.075 C. $31 \geq 0$ 0.4.654 0.6125

$10.000 .0750 .11200 .46 \leqslant 4 \quad 0.1225$

$12.00 \quad 0.0750 .21200 .5+34$ C.C125

$2.00 \quad 0.0750 .01200 .9962 \quad 0.0125$

$4.00 \quad 0 . C 75 \quad C .0 .1200 .996$ C.

0.00 C.C.75 $0.01 \geq 0.0 .44<=0.6125$

1.00 C.175 0.71?2 0.99ez C.L1?5

10.04 C.475 0.0120 u.9.9C.. C.(12)

12.00 C.. 75 U. 1120 0.4948 C. $12 \mathrm{~m}$
0.1810

0.2913

4.3544

i. 4312

ن. 5648

0.6548

0.1454

0.2316

U. 3074

0.3777

$0.444 t$

U. 5093

$0.12 \% 0$

0.2059

0.2722

0.3333

0.31310

0. 4475

0.1214

0.1923

0.2537

$0.31 J 3$

0.3640

0.4133

4.1172

$0.185{ }^{\circ}$

ن. 2444

i. 2106

0.3479

v. 3942

c. 1160

$0.10 j 2$

0.2416

w. 2.122

c. 3454

$0.324=$
0.2102

0.3257

0.4116

$0.4 \$ 13$

0.2388

0.2901

0.3157

0.4980

0.0360

0.7511

0.0483

$0.932 t$

0.3924

0.0232

0.0030

0.9533

1.0785

1.1918

0.4463

0.7117

0.1203

1.0440

1.2430

1. 2762

0.4775

0.7055

0.1303

1.1735

1.0423

1.4057

0.4673

$0.7 \pm 14$

L. Uं10

1.2055

1. 3714

1. $2: 80$
0.5298

0.9116

1. 00 i 0

1.1)100

$1.00 \cup C$

2. ن Ou 0

0.5298

0.9116

1. 0000

1. Juvo

1. cuvo

1. 0000

3.3240

0.9116

1.0000

1.0000

1.0000

1.0000

0.5298

0.9116

1.0000

1.0000

1.0000

1.0000

0.5248

$0.9: 16$

1.0000

1.0000

1.0000

1.01000

0.5245

0.3116

$1.00 \cup 0$

1.00 .10

1.0000

1.0000
4र. 8450

5 ट.70'

54.2423

$41.020 \mathrm{~s}$

34.0 .3 .14

26.0795

46.4372

50.2478

57.9425

SI. 007 .

44.2647

30.5190

$40.27 i 1$

EU. 7 ios

OL. 2nOJ

52.41:0

4y. シ U4ग

44.0674

49.5510 oc. 0371

03.5033

57.7351

इ८. 22<J

47.1024

5u.1355 $0<.7104$

64.4575

20.1307

23.5323

$4 C .25=1$

54.3742

$6<.3 .101$

64.7300

54.21 .7

24.7231

4บ. 
FLOH CIA.

MANN. COEFF
SUPPLY CIFAIN

SLOPE SLUPE (SIN) (SIN)
DP.AIN FLOH

ENTRY OEPTH

RATIO H/O.
Eattōy

ENERGY

H.
NORMAL

DEPTH

H/D.
PIPE LENCTH T

MORAIAL DEPIH. LIO.

$\begin{array}{rllll}2.00 & 0.075 & 0.0150 & 0.2586 & 0.0500 \\ 4.00 & 0.075 & 0.0150 & 0.2586 & 0.0500 \\ 6.00 & 0.075 & 0.2150 & 0.2506 & 0.4500 \\ 8.00 & 0.075 & 0.2150 & 0.2588 & 0.1500 \\ 10.00 & 0.075 & 0.0150 & 0.2568 & 0.0500 \\ 12.00 & 0.075 & 0.0150 & 0.2588 & 0.0500\end{array}$

$2.00 \quad 0.075 \quad 0.0150 \quad 0.5$ ÜCC 0.0500 $\begin{array}{lllll}4.00 & 0.075 & 0.0130 & 0.5 C C O & 0.0531 j\end{array}$

$6.00 \quad 0.0750 .02500 .5000 \quad 0.0500$

$8.00 \quad 0.0750 .0150 \quad 0.5600 \quad 0.5590$

$10.00 \quad 0.075 \quad 0.32500 .5 C 0 C \quad 0.05100$

$12.00 \quad 0.075 \quad 0.0250 \quad 0.5 C C C \quad 0.0500$
0.2103

0.3411

0.4507

0.5695

0.6753

0.7806

0.2606

0.2703

0.3640

0.4446

0.5249

0.6040

0.1503

0.2349

0.3106

c. 3926

0.4617

0.5228

0.1405

0.2238

0.2906

0.3640

0.4200

0.4897

0.1356

0.2155

0.2054

0.3449

0.4114

0.4705
0.1614

0.2471

0.3100

0.3502

0.4029

0.4391

0.2394

0.3732

0.4730

0.5549

0.0240

0.0611

0.2966

0.4659

0.5952

0.7008

0.7904

0.8692

0.3369

0.5316

0.6313

0.5053

0.9116

1.0038

0.3605

0.5714

0.7334

$0.26=5$

0.9827

1.0833
0.3743

0.6284

0.8647

1.0000

1.0000

1.0000

0.3743

0.6284

0.8647

1. 0000

1.0000

$1.00 J 0$

0.3743

0.6284

0.8647

1.0000

1.0000

1.0000

0.3743

0.6254

0.8647

1.0000

2. 0000

1. 0000

0.3743

0.6254

0.8647

1. 0000

1.0000

1.0000

0.1342

0.2133

0.2520

0.3459

U. 4uos

0.4051
0.3681

0.5327

0.7505

0.5374

1. 0054

1.1073
0.3743

0.0284

0.8647

1.0000

1.0000

1. 0000
25.2919

36.4966

40.9254

34.1350

21.5611

24.2041

28.1506

43.0225

52.7245

41.1453

24.3543

22.6614

29.3730

45.0014

$520 \$ 296$

44.3432

33.0452

26.7842

30.0179

40.0559

50.7205

40.1728

35.0805

29.17425

30.33 .45

46.6006

57.4335

47.3947

30.1250

30.1373

30.4343

46.7449

57.6573

47.3500

30.4204

30.5 .570 


FLOH OIA. MANN. SUPFLY CPAIN
LIS. F. CJEFF SLOPE SLGPE
(SIA)

$2.00 \quad 0.4750 .0150 \quad 0.2586 \quad 0.4250$

$\begin{array}{lllllll}4.00 & 0.075 & 0.3150 & 0.2586 & \text { C. } & 0.350\end{array}$

$6.00 \quad 0.075 \quad c .3150 \quad 0.2586 \quad 0.0250$

$8.00 \quad 0.075 \quad 0.1) 150 \quad 0.256 t \quad c . C 550$

$10.00 \quad c .675 \quad 0.3150 \quad 0.25$ t t $\quad c .0 ? 50$

$12.00 \quad 0.6750 .3150 \quad 0 . \bar{c} 540 \quad$ C.C253

$2.00 \quad 0.0750 .3150 \quad 0.50100 \quad 0.0250$

4.00 C.C.75 0.0150 0.50CU C.C250

$6.00 \quad 0.675 \quad C .0150 \quad 0.5 C O C \quad 0.6 .250$

8.00 C.C75 0.01>0 3.50CE n.U250

$10.00 \quad 0.075 \quad 0.3150 \quad 0.5 C C_{U} \quad 0 . C \geq 50$

$12.00 \quad 0.0750 .31530 .5 C C L$ C. 0250
DKAIN FLUH

FNTKY DEPTH

RAT $10 \mathrm{H}$ HL.
ENTRY

ENERGY

M.
MORMAL

DEPTH

H/D.
PIPE LEISTH II NOFMAL UEPTH. L/O.

\begin{abstract}
0.2103
0.3411

0.4587

0.5648

0.6703

0.7800

0.1056
0.2703
0.3606
0.4446
0.5249
0.0040
\end{abstract}

0.1503

0.2349

0.3100

$0.39: E$

U. 4617

0.5290

0.1405

0.2236

0.2706

$\checkmark .3040$

0.4200

$0.48+7$

C.1350

0.2155

c.. 2354

ن. 3443

0.4114

6.4705

0.1342

0.2133

0.2520

c. 3454

v.4.jes

0.4052
0.1614

0.2471

0.3100

0.3602

0.4029

0.4371

0.2394

0.3731

0.4730

0.3549

$0.0<40$

0.0811

0.2966

0.4659

0.5952

0.7000

0.7404

0.0692

0.3360

0.5315

0.0213

0.6053

0.1116

1. $003 y$

0.3605

0.5714

0.7334

0.7085

0.9427

1.0033

0.3681

0.2027

0.1503

0.1874

1.0054

1.1073
0.4832

0.8257

1. COUU

1.0000

1. 0000

1.0000

0.4032

0.8257

1.0000

1.0000

1. 0 บัن 0

i. 0000

0.4632

0.8257

1.0060

1. 0000

$1.00 \cup 0$

1.0000

0.4332

0.0257

1. 0000

i. 0000

1.0000

1.0000

0.4832

0.8257

1.0000

1.0000

1.0000

1. 3000

$0-4832$

U. 6227

1. 0000

1.0000

1.0000

1. DOu ú
27.6673

$37.37>0$

31.7501

$23.51<5$

17.5443

12.1473

30.2307

41.5546

37.2575

30.1712

24.5314

20.1304

31.4041

45.4919

39. jगय

$33.2=37$

$20.33=1$

24.0525

32.0373

44.5470

41.2502

34. 450 ?

30.3131

$20.20=5$

32.3901

$4=.0910$

$4 \angle 0 \cup \cup 10$

3) $0=2$

3i. $312 i$

C7. 547

32.4317

43.2444

4.. 2207

$30.1 \% 3$

j.

27.0170 
FLOM CIA. MANN. LSS. H. COEFF
SUPPLY CPAIN

SLOPE SLOPE

(SIN) ISIN]
DRAIN FLOH

DRAIN ELOH ENTRY

RATIO HIO.
ENTRY

ENERGY

Mo
NORMAL DEPTH

H/D.
PIPE LEITCR.A NORAAL DEPTHA

L/O.

\begin{abstract}
$2.0060750 .3150 \quad 0.25860 .0167$
$\begin{array}{lllllll}4.00 & 0.075 & 0.0150 & 0.2588 & 0.0267\end{array}$

$6.00 \quad 0.0750 .0150 \quad 0.2566 \quad 0.0167$

$8.00 \quad 0.075 \quad C .0150 \quad 0.2568 \quad .0167$

$10.00 \quad 0.075,0.03500 .258 E$ C.C. 167

$12.00 \quad 0.0 .750 .5: 50 \quad 0.2588 \quad 0.6167$
\end{abstract}

$10.00 \quad 0.0750 .0150 \quad 0.7670 \quad 0.0167$

$12.00 \quad 0.075 \quad 0.0150 \quad 0.7070 \quad 0.0137$
0.2103
0.3411
0.4507
0.5698
0.6703
0.7500

0.1680

0.2703

0.3606

0.4446

0.5249

0.6040

0.1503

0.2399

0.3166

0.3910

0.4617

$0.528 \varepsilon$
0.1405

0.2230

0.2906

$0.364 \mathrm{C}$

0.4200

0.4897
0.1614
0.2471
0.3100
0.3602
0.4029
0.4391

0.2394

0.3731

0.4730

0.5549

0.0240

0.0811

0.2966

0.4654

0.5952

0.7005

0.7904

0.0092

0.3369

0.5316

0.0513

0.3053

0.4126

1.0038

$0.135 h$

U. 2L5 5

0.2354

0.3499

0.4114

0.4705
0.3605

0.5714

0.7334

0.06685

0.9027

1.0833
0.5630

0.9712

1.0000

1.0000

2.0000

1.0000

0.5630

0.9712

1. 0000

1.0000

$1.00 \overline{0} 0$

1.0000

0.5630

$1.00 \div 0$

1.0000

1. 0000

1.0000

0.5630

0.9712

1.0000

1.0000

1.0000

1.0000
0.9712

23.0455

23.4572

27.1040

21.7743

10.5550

11.6637

25.6075

25.2314

32.4483

$20.10 y 4$

23.6340

14.4251

26.0014 22.2852 35.0008 31.1552 27.1433 23.3270

27.4506

34.7112

36.40331

32.8464

24.09 .2

25.4914

27.7777

72.5463

$37.13: 1$

$33.72=1$

$30.03 \% 0$

20.5443

0.5630

$0.97 i 2$

1.0000

1.0000

2.0000

1.0000
$27.57=9$

$57.7 \div 40$

37.3542

33.4739

30.3 .541
20.9003 


FLOW [IA. MANN. SUPPLY ISAIN
LIS. M. COEFF SLCPE SLUPE
(SINJ (SIM)

ORAIN FLOH

ENIRY DEPTH

RATIC H/C.
ENTRY

ENERGY

M.
MORMAL OEPTH

H/D.
PIPE LEITCTH TC NORMAL JEPTH. L/J.

\begin{abstract}
$2.00 \quad 0.075 \quad 0.0150 \quad 0.25 t t \quad 0.0125$
4.0u $0.075 \quad 0.0150 \quad 0.2548 \quad 0.0125$

6.00 C.C75 $0.3150 \quad 0.25600 .0125$

$9.00 \quad 0.075 \quad 0.0150 \quad 0.25 t c \quad 0.0125$

$10.00 \quad 0 .(75 \quad 0) 150 \quad 0.25 t=$.

12.000 .0750 .01500 .25 te 0.0125
\end{abstract}

$2.00 \quad 0.075 \quad 0.01500 .5 \operatorname{ccc} 0.0125$

$4.00 \quad 0.675 \quad 0.1) 1500.5 C 0(\quad . C 125$

6.00 C.C.75 0.3150 0.5000 0.0125

n.00 i.c. 75 c.)1jo 0.5000 C.U125

$10.00 \quad c .0750 .1) 1500 . \pm C(C$ C. C125

$\$ 2.000 .0750 .71 .500 .5 C 60$ C.LI?5

\begin{abstract}
0.2103
0.3411

0.4567

0.5690

0.0703

C. $750=$
\end{abstract}

0.1000

0.2703

0.36 ن̃o

0.4446

C. $524 t$

0.6040

0.1503

0.2399

ن. 3100

0.31318

0.4517

0.5208

0.1405

0.2238

c. 2960

0.3640

ن. 4200

0.4397

0.1350

0.2155

0.2054

U. 34 .

C. 4124

l. 4765

0.1342

0.2133

0.2020

U. $34>9$

[. 400 b

U. $40>1$

\begin{abstract}
0.1614
0.2471

0.3100

0.3602

0.40029

0.4391
\end{abstract}

0.2394

0.3731

$0.4731)$

0.3544

0.0240

0.0811

0.2966

0.4057

0.3452

0.7003

0.7404

0.0692

0.3364

0.2310

0.0013

0.0053

0.9110

1.0030

0.3605

0.5714

0.7334

0.0685

$0 . y=27$

1. 0333

0 - उढ त I

0.3827

0.7205

0. yc 74

1.นن̀4

1. 1073
0.6204

1.0000

1.00 .0

1.0000

1. 0000

1. 0000

0.6204

1. 0000

$1.000 \mathrm{us}$

1.0000

$1.00 \cup 0$

1.0000

$0.62 E 4$

1. $00<0$

1.0000

1.0030

1.0000

1.0000

0.6204

1. 0000

1.0000

1.0000

1.0000

1.0000

$0 . t 204$

1.0000

1.0000

1.0000

1.0000

1.0000

0.6204

1.0000

1.0000

1.0000

1.0000

1.0000
$0.5>77$

22.6261

25.4236

20.4417

10.1022

11.4143

10.9101

20.7413

$30.70 \div 5$

27.21573

25.1525

1).0y, 7

17. 49 t5

28.7257

33.2500

30.2323

20.5855

$2<.9502$

$18.340 \mathrm{~J}$

29., 342

34.5340

31.7140

$28.53=7$

$25.11+3$

19.1724

30.4167

35.3537

$3<.7+-3$

24.51 il

20.2177

14. 5473

$30.57: 7$

32.:2 24

3ز.017נ

24.02द

26. 5? 3 
FLOH DIA. MANN SUPPLY DKAIN LIS. M. COEFF SLOPE SLUPE (SIN) (SIN)
DRAIN FLOH ENTRT DEPTH RATIO AID.
ENTRY

ENERGY

M.
NORMAL UEPTH HIO.
PIPE LENETH I NORMAL UEPTH. L/O.

$\begin{array}{rllll}2.00 & 0.075 & 0.0130 & 0.2586 & 0.0500 \\ 4.00 & 0.075 & 0.11110 & 0.2580 & 0.0500 \\ 6.00 & 0.075 & 0.1130 & 0.2580 & 0.0301) \\ 8.00 & 0.075 & 0.0130 & 0.256 E & 0.0500 \\ 10.00 & 0.075 & 0.0130 & 0.2584 & 0.0500 \\ 12.00 & 0.075 & 0.0130 & 0.2566 & 0.050 J\end{array}$

$2.00 \quad 0.0750 .01300 .50000 .0500$

$4.00 \quad 0.0750 .0130 \quad 0.5000 \quad 0.0500$

$6.00 \quad 0.0750 .01300 .5 c 000.0500$

$8.00 \quad 0.0750 .3130 \quad 0.5 C C .0 \quad 0.6500$

$10.00 \quad 0.075 \quad 0.31300 .5 C C C \quad C .6500$

$12.00 \quad 0.0750 .31300 .5000 \quad 0.0500$

$2.00 \quad 0.075 \quad 0.31300 .7070 \quad 0.0500$

$4.00 \quad 0.0750 .31300 .7670 \quad 0.6500$

$6.00 \quad 0.075 \quad 0.2130 \quad 0.767 C \quad 0.05000$

$8.00 \quad 0.075 \quad 0.01 .10 \quad 0.7070 \quad 0 . C \equiv 00$

$10.00 \quad 0.0750 .3130 \quad 0.70 .7 \mathrm{C} \quad 0.0530$

$12.00 \quad 0.075 \quad 0.71300 .7070 \quad 0.1500$

$2.00 \quad 0.075 \quad c .0190 \quad 0.8660 \quad c .0500$

$4.00 \quad 0.075 \quad C .01300 .8 .660$ C.C.500

$6.00 \quad 0.0750 .01300 .8 E \in C \quad C .0500$

$8.20 \quad 0 . c 75 \quad 0.7130$ 0.8c. o c.cisno

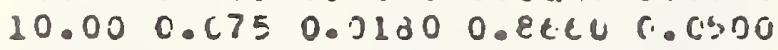

$12.00 \quad 0.075 \quad 0.31300 .8 t 60 \quad$ C.C5nO

$2.00 \quad 0.0750 .01300 .96 .590 .0500$

$4.00 \quad 0.075 \quad 0.0130 \quad 0.9659 \quad 0.0500$

6.000 .0 .750 .01500 .90 .59 C. 0500

$8.00 \mathrm{C.075} 0.01300 .5059 \mathrm{C.U} .00$

$10.00 \quad 0.0750 .0130 \quad 3.9659 \quad 0.0500$

$12.00 \quad 0.075 \quad 0.121300 .4059 \quad 0.0500$

0.2334
0.3844
0.5254
0.6540
0.7500
0.9028

0.1906

0.3074

0.4114

0.5093

0.6040

0.6940

0.1696

0.2722

ن. 3630

0.4475

0.5268

0.6079

0.1586

0.2537

0.3372

0.4123

$0.4 B 77$

$0.562 U$

0.1530

0.2444

0. 3245

0.31i72

0.4705

U.5376

0.1512

0.2416

0.3210

$0.394=$

0.4651

0.5337
0.1312

0.1992

0.2491

0.2396

0.1220

0.3523

0.1917

0.2959

0.3735

0.4357

0.4468

0.5326

0.2368

0.3682

0.4672

0.5484

0.0157

0.6733

0.2682

0.4196

0.5354

0.0289

0.7083

0.7705

0.2269

0.4499

0.5752

0.5770

0.7631

0.0373

0.2930

0.4595

0.2867

0.0911

0.7796

0.3544
0.4230

0.7241

1. 0000

1. 0000

1.0000

1.0000

0.4280

0.7241

1.0000

i. 0000

1.0000

1.0000

0.4250

0.7241

1.0000

1.0000

1.0000

1.0000

0.4280

0.7241

1.0000

1.0000

1.0000

1.0000

0.4200

0.7241

1. $00 \cup 0$

$1.00 v 0$

1.0000

1. 0000

0.4200

0.7241

1.0000

1.0000

1. 0040

1.0000
16.0963

25.8504

32.1132

$15.80,3$

10.1405

3.7500

20.1423

29.0704

30.3144

20.5052

13.4670

11.3412

21.0036

30.5722

36.2032

22.9777

10.1600

14.4672

21.5575

31.3847

40.1704

24.2404

$14.667 \%$

10.1354

21.8024

$3 i .7402$

40.5321

24.4052

CU. 433.5

16. $7=27$

21.0540

31.7210

410 0ंस

23.1514

20.0302

17.2071 


FLOH DIA. MANN. SUPPLY OFAIN
LIS. P. CIJFFF SLIPE SLUPF
(SINI SINI

DHASIN FLCH

ENTRY OSPTH

KATIO HIO.
ENTEY

ENERGY

i.
NORMAL

UEPTH

H/J.
PIPE LENGTH TL NORMAL JEPTH. L/D.

\begin{abstract}
$2.00 \quad 0.075 \quad 0.0130 \quad 0.2568 \quad 0.0250$
$4.00 \quad 0.675 \quad 0.7130 \quad 0.252 t \quad C . C>50$

$6.00 \quad C .075 \quad 0.3: 300.252$ C $\quad .0 .250$

$4.00 \quad 0.075 \quad 0.3130 \quad 0.5580 \quad(1.25 !)$

$10.00 \mathrm{C.c75}$ c. $) 1+0$ 0.z5t大 C.c250

$12.00 \quad 0.075 \quad 0.2130 \quad 0.254 t \quad 0.0250$
\end{abstract}

$2.000 .075 \quad c .31300 .5 C C C$ r.0.50

$4.000 .(75=0.31300 .50 C$ C.C.25U

$0.00 \quad 0.0 .75 \quad 0.11350 .5 \operatorname{coc}(.0 .0250$

$8.000 .0750 .71: 00 . \sec c .6250$

$10.00 \quad c . c 75 \quad 0.7130 \quad 0.5 C C<0.0250$

$12.000 .075 \quad 0.01330 .5060$ C..2250

$2.00 \quad 0.0750 .21300 .76 .70 \quad 0.0250$

4.00 C.C.75 $0.13300 .7 C 7 C$ C. 0755

6.00 C.C75 $0.31300 . \overline{T C 7 C}$ C.0.230

$3.00 \quad 0.075 \quad c .3130 \quad 0.7076 \quad c .4255$

$10.00 \quad 0.6750 .01300 .7 C 7 C \quad .0250$

12.000 .0750 .3130 n.747L C.C250

2.000 .0750 .01100$. etec $\quad$ C.02 00

4.00 C.L75 0.313.j C.etcC C.CZ5.

6.00 C.CT5 0.J130 o.tetC r.L $\geq 50$

3.00 C.C7E 0.0130 0.8tel $\quad .1250$

$10.0, C . C 750.31330$. tete C.C250

12.000 .0750 .2130 O.feCL 0.4250

2.00 C.C.75 0.3130 2.9t5y C.C?50

$4.00 \quad .075 \quad$ c. $) 1100.9 t 59 \quad(.0250$

0.00 C.C75 c. 3190 j.c.e 54 C.1 250

$\because .00 \quad(.0750)$.1 , is $0.4 t=4 \quad 0.050$

10.00 C.c75 0.1150 0.re.

$12.00 \mathrm{C.075} 0.01800 .58 .54 \quad 0.6 ? 50$

$2.00 \quad 0.075 \quad(.01300 .94 \mathrm{Cs} \quad .0 .250$

4.00 0.C.75 0.111.) 0.19le C.C?511

$0.00 \mathrm{C.075}(0.01+) 0.4402 \quad .0250$

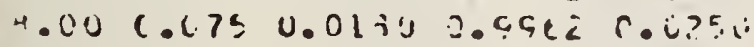

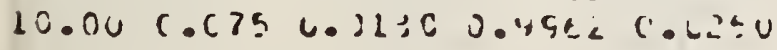

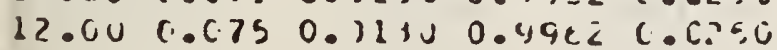

0.2364

0.3594

C. $.52>9$

0.6540

0.7048

0.9020

0.1900

c. 3074

$6.41: 4$

$0.50 \div 3$

0.6040

0.6945

0.1690

c. $272:$

C. 3530

0.4475

v. 5200

0.607 i

0.1540

ט.2037

0.3372

0.4153

v. 4397

v. 562 U

C. 1530

0.2444

v. 3245

U. 3432

0.4705

U.5340

C. 1512

v. 2410

c. 3210

U. 3740

U. 4031

U.33
0.1312

0.1972

$0 .<491$

0.2895

0.3228

0.3523

0.1917

0.2959

0.3735

0.4357

0.4 .668

0.2325

$0 . \angle 366$

0.3642

0.4672

0.34 स 4

0.0157

0.0733

0.2682

0.4190

0.3354

0.bits

0.7023

0.7765

0.256 .9

0.4449

0.2752

0.6770

0.7631

0.0173

0.2930

0.4540

0.0007

0.0911

0.1734

0.0544
0.5542

0.7596

1.0000

1.0000

1. 0000

1. 0000

0.5542

ن. 9530

1.00 .00

1.0000

1.0015

1.0000

0.5542

0.4536

i. 0000

1.0000

1.0000

1.0000

0.5542

0.9520

1.0000

1.0000

2.000

1. วงว०

J. 3542

1.9 920

1.00u

$1.0 \mathrm{~J}, 0$

1. 0000

1.00u's

0.5542

U. 9536

1. U() 0

1. C.Ju

1. यJu

1.0000
13.5035

$14 .=241$

10.37.3

$1<.7403$

$0.7=51$

2.1041

17.4220

20.4574

CU.74 4

17.2325

13.7744

1U.je 3

10.3434

21.0503

$22.04>3$

14.5124

$10.30>5$

13. उपे?

$10.34 J 4$

21.5715

23.7237

24.7350

17.32 .0

12.0123

$140.8 \% 5$

$210: 410$

$24.27 \mathrm{~J}$

21.4931

1.

12. $=373$

14.17:0

$21.11 ! 2$

$24 \cdot+1: ?$

$21.0<04$

is. 07711

16. 1) $3 . .1$ 
FLOH DIA. MAMY SUPPLY DRAIN LIS. H. COEFF SLOPE SLOPE (SIN) ISIN)
DRAIN FLOH

EMTRY DEPTH ENERGY

RATIO MID.
EHTRY

H.
NORMAL DEPTH

HOD.
PIPE LEYGTH T MORMAL JEPTH. L/O. $\checkmark$

$2.00 \quad 0.0750 .0130 \quad 0.25880 .0167$

$4.00 \quad 0.075 \quad 0.0130 \quad 0.25480 .0167$

$6.00 \quad 0.075 \quad 0.0130 \quad 0.2568 \quad 0.0147$

$6.00 \quad 0.0750 .11130 \quad 0.25 E 2 \quad$ C.0167

$10.00 \quad 0.0750 .11100 .25 E 50.41 h 7$

$12.00 \quad 0.0750 .31300 .25 E$ C.U167

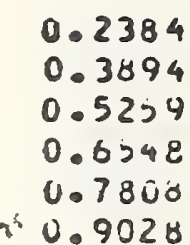

0.1906

0.3074

0.4124

0.5093

0.6040

0.6948

$0.16 \cdot 16$

$2.00 \quad 0.0750 .01400 .70760 .0167$

$4.00 \quad 0.075 \quad 0.01300 .7070 \quad$ C.ulh?

$6.000 .075 \quad 0.71300 .7 c 700.4157$

$6.00 \quad 0.075 \quad 0.31300 .7 C 7 C \quad 0 . C 167$

$10.00 \quad 0.0 .75 \quad 0.31300 .7070 \quad 0.0157$

$12.00 \quad 0.0750 .01300 .7070 \quad 0.0167$

$2.00 \quad 0.075 \quad 0.0130 \quad 0.8 t 60 \quad 0.0267$

$4.00 \quad 0.075 \quad 0.01300 .8 t 60 \quad 0.0157$

$6.00 \quad 0 . C 750.01300 . \varepsilon t \in U$ C.C.107

$8.00 \quad 0.075,0.31300 .86 t 0 \quad 0.0167$

10.00 C.C.75 C. . 130 O.tetc C.clis

$12.00 \quad 0.0750 .71300 .8600 \mathrm{C.0167}$

0.4897

0.5620
0.1506

0.2537

0.3372

0.4253

0.1530

0.2444

0.3245

0.3942

0.4705

0.5340
0.1312

0.1992

0.2491

0.2890

0.5228

0.3523

0.1927

0.2959

0.3735

0.4357

0.4868

0.2325

0.2368

0.3682

0.4672

0.5484

0.0157

0.0733
0.6470

1.0000

1.0000

1.0000

1.0000

1. 0000

0.6470

1.0000

1.0000

1.0000

1. 0060

1. 0000

0.6470

1.0000

1.0000

1.0030

1.0000

1.0000

0.2682

0.4196

0.5354

0.0289

0.7083

0.7765
0.6470

1.0000

1.0000

1.0000

1.0000

1.0000

0.6470

1.0000

1. 0000

1.0000

1.0000

1.0000

$2 \cdot 4537$

13.9947

25.3240

12.01io

0.4264

$5.011 j$

8.8134 16.8502 19.1147 10.4507 13.3035 10.3037

9.2045 10.4214 20.9441 10.6545 15.3610 13.0784

9.3122 14.2442 22.0031 19.9831 17.3023 14.6792

9.3341 14.0003 22.0050 20.5030 18.0410 $15.497 \mathrm{~J}$

C. 1512

0.2410

0.3210

0.3448

$0.46>1$

0.5337
0.2930

$0.4 \leq 98$

0.3867

0.0911

0.7790

0.0544
0.6470

1.0000

1.0000

1.0000

1.0000

1.0000 y. 3346 14.7904 $26.74+6$ 20.7450 10.2933 15.7141 


$$
D=0.10
$$


FLOH DIA. LIS. M.
RANH. SUPPLY ORAIM

CUEFF SLCPE SLCPE

(SIN) (SIN)
ORAIN FLOH

ENTRY LIEPTH

PATIO H/D.
ENTRY

ENERGY

M.
NURMLL

UEPIH

H/O.
PIPE LENGTH

NOKMAL JEPT

L/D.
$2.00 \quad 0.2 C C \quad 0.33100 .25420 .0500$

$4.00 \quad 0.100 \quad 0.3020 \quad 0.2566 \quad$ C.0500

6.00 C.10C C. 0370 0.258E 0.0300

$8.00 \quad 0.100 \quad 0.33300 .2586 \quad$ c.0500

$10.00 \quad c .1 C 0 \quad 0.1090 \quad 0.25 t b \quad c .0500$

$12.00 \quad 0.1$ UC $0.0010 \quad 0.25600 .0500$

$2.00 \quad 0.10 C \quad 0.3070 \quad 0.5 C C C \quad 0.0500$

$4.00 \mathrm{C.ICC} 0.2390 \mathrm{0.5CCL}$ C.6500

$6.00 \quad 6.100 \quad 0.30700 .2000 \quad 0.0500$

$8.000 .1 C C \quad 0.30700 .5$ cuu C.. 1.500

$16.00 \quad 0.2 C 0 \quad 0.3030$ J.sccic 0.0500

12.000 .1000 .03100 .800 C. 0500

$2.00 \quad c .10 C \quad 0.2090 \quad 0.7 C 7 C \quad C .6500$

$4.00 \quad c .100 \quad 0.3070 \quad 0.707 \mathrm{C} \quad \mathrm{C.050C}$

6.00 $\quad 0.106 \quad 0.70 .70 .7676 \quad 0.0 .500$

$8.00 \quad 0.1000 .00100 .707 C \quad 0.0500$

$10.00 \quad C .100 \quad 0.3070 \quad 0.7 C 7 C \quad 0.0530$

$12.00 \quad 0.100 \quad 0.3070 \quad 0.70700 .0500$

$2.00 \quad 0.1 C C \quad 0.30300 .8 t 60 \quad c . c 5 n 0$

$4.00 \quad 0.100 \quad 0.02700 .860 C \quad 0.0500$

$0.00 \quad 0.10 C \quad 0.30300 . \operatorname{tot} C \quad C .6530$

$8.00 \quad 0.100 \quad 0.0030 \quad 0.8606$ c.0500

$10.00 \mathrm{C} .1000 .00300 .060 \mathrm{C}$ C.0500

$12.000 .100 \quad 0.3 .3100 .86 e c \quad c .0500$

$2.00 \quad 0.100 \quad 0.6270 \quad 0.9059 \quad 0.050 ! 5$

$4.000 .1 C 0 \quad c .02100 .5 t 54$ C.usno

$6.00 \quad 0.10 C \quad 0.03700 .4 t^{5}=y \quad$ C. $45 n 0$

$8.00 \quad 0.100(0.1) 1) 0.9+54$ C.(.5.2n

$10.00 \quad 0.100 \quad 0.17 n 20 \quad 0.46 \leq 4 \quad \operatorname{cocs} ? 0$

$12.00 \quad 0.1000 .70720 .5854 \quad 0.0500$

$2.00 \quad 0.100 \quad 0.50 .300 .99 t 2$ c.c5no

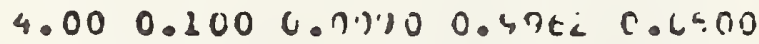

6.0u C.1CC $0.30100 .491 .20 .65 n 0$

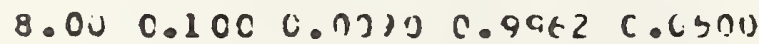

$10.00 \quad 0.100 \quad 0.30100 .44$ ce $C .0=00$

$12.000 .160(0.3 .770$ 0.94e. C.051)

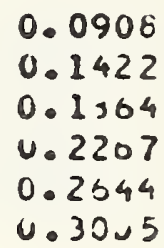

0.0737

$0.114 \varepsilon$

0.1445

0.1313

0.2108

0.2304

0.0660

0.1026

0.1334

0.1615

0.1374

0.2120

0.0620

0.0901

0.1244

0.1510

0.1752

0.1479

0.0505

$0.09 i \varepsilon$

0.1205

0.1450

0.1 .334

U.140

0.0543

0.0914

0.11 .3

0.1442

0.1071

0.1008
0.2566
0.4174
0.5467
0.4575
0.7555
0.8426

0.3830

0.6302

0.0355

1.0108

1.1679

1.3099

0.4749

0.7849

1. 4441

1.2063

1.4704

1.0537

0.5374

0.0921

1. 1591

1.4457

1.0785

1.5942

0.5752

0.7555

1.2748

1.3526

$1 .=052$

2.0352

0.5858

0.1757

1. 3000

1.5834

1.0414

2.0775
0.1549

0.2478

0.3243

0.4050

0.4775

0. 5403

0.1549

0.247 e

$0.32 \div 3$

0.4050

0.4778

0.5403

0.1549

0.2473

$0.32+3$

0.4000

0.4778

0.5463

0.1549

0.2475

0.3243

$0.40=0$

0.4775

$0.54 c 3$

0.1549

0.2478

0.3243

0.4050

0.4778

0.5403

0.1549

0.2470

0.3243

0.4030

0.4778

0.5453
34.5374

56.9455

$76.3=11$

94.2911

104.7754

$124.07 \times 4$

$30 \cdot 3270$

63.7402

ò 7.4115

105.3044

122.5377

$13 c .44 J 0$

40.5040

60.3774

90.9337

109.3459

127.5413

144.2125

41.2825 69.7013

$92.7 \div 24$

$111.57=0$

130.2103

147.1742

41.0777

7ט

93.2111

112.5935

$131.53+7$

$146 .: 3,3$

$41.77 \div 0$

76.5521

91.53 .3

113.29. 7

131.0431

$144.141:$ 
FLOH DIA. MANY
LIS. . M. CJEFF
SUPPLY CKAIN

SLUPE SLLPE

(SIN) (SIPI)
ORLIN FLOH

ENTRY DEPTH

QATIO H/L.
ENTRY

ENERGY

M.
MOKMAL UEPTH

H/U.
PIPE LEINCTA TO MOKMAL JEPTH. L/D. $\begin{array}{llllll}2.00 & 0.100 & 0.00 .70 & 0.2568 & 0.1250\end{array}$ $4.00 \quad 0.100 \quad 0.23+2 \quad 0.251: 6 \quad 0.02<0$ $6.00 \quad C .100$ c. $0.7+0 \quad 0.2 \leq 00 \quad C .0 \leq 50$

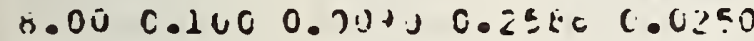
$10.00 \quad 0.100 \quad 0.30 .10$ ?.zEEc C.L.251 $12.00 \quad 0.160 \quad 0.3 n>0 \quad 0.25 E C \quad C . C 250$

$2.000 .1 C 0 \quad 0.00700 .50 C 6(.0 .0250$ $4.00 \quad 0.100 \quad 0.3070 \quad 0.5 C C_{i} \quad C .0 .250$ $6.00 \quad 0.1(6)$ C. . ग) 6.00 C.100 0.3010 0.5C0C C.. 250

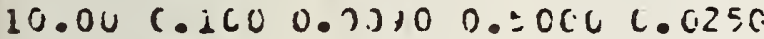

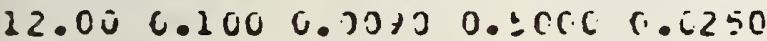

$2.00 \quad 0.160 \quad 0.30+0 \quad 0.707 c$ r.c.250 $4.00 \quad 0.100 \quad 0.20700 .7 C 7 C \quad c .6250$ 0.00 C.1CC $0.307 . J 0.7 C 7 L$ C.L.2 50 $3.00 \quad 0.10 \mathrm{C} 0.33+00.707 \mathrm{C}$ C.C250 10.0 u $0.100 \quad 0.30300 .7(.7$ u C.C250 $12.00 \mathrm{C.1CC}$ C. $10300.7 \mathrm{C} 7 \mathrm{U}(\ldots 250$

0.0908
$v .1422$
6.1364
$i .2207$
$\because .0644$
0.3005

0.0737

U. 1248

U. 1445

‥ 1913

U. $21 v=$

i. 23ie

0.0600

0.1126

0.1334

0. 1613

i. 1374

U. 2120

0.0620

0.0701

U. 124 ?

0.1510

U. 1722

0.1 .37 .3

$0.054 \mathrm{~s}$

$0.096=$

C. 1205

i. 1400

U. $10, y$

0.1 two

$0.05+3$

0.0724

U. 11.3

U.:244i

0.1071

veidis
0.2560
0.4174
0.3467
0.0575
0.7555
0.0420

0.3830

0.6302

0.5355

$1.010 j$

1.1679

1.3 .099

0.4749

0.7049

1.6441

1.2660

1.4704

1.0537

0.2374

0.0921

1. 1491

1.4457

1.0785

1.5942

0.2752

$0.4>55$

1. $\angle 748$

1. \320

1. 0052

2. 0352

0.5458

$0 .+757$

1.30 40

1.2334

1. $141 \mathrm{~J}$

$2.077 v$
0.1934

0.3157

ن. 4231

0.3239

$0.6 \angle i 6$

0.7103

0.1954

0.3157

0.4232

J.5239

3.0216

ن. 7103

0.1954

0.3157

ט. 4231

0.5239

0.0216

0.7103

0.1924

U. 3157

0. 4231

0.5239

0.6216

0.7103

ن. 1954

0.3127

0.4231

0.5234

0.0216

0.7103

0.1954

0.3127

0.4231

0.5234

j.ं $<10$

ง. $71: 1$
40.2220

79. तम2.3

103.3503

124 . 16j5

$14<.42+J$

150.4177

51.3902

84.7493

111.6313

133.5 .375

153.0321

17 . $130 \mathrm{~s}$

53.0057

E7.15 3

114.37 .0

137.0047

150.0137

176.3345

$53.72+2$

80.41523

110.50:1

134. 71:1

101.0084

174.0773

54.09134

84. 1.23

117.41 .4

$14 \mathrm{v} .70 .0$

$10 \therefore .34 .1$

$130.44=3$

54.1452

e4. 316,

$111.65,2$

14i...13s

$10<\cdot 0+1$

$1 \mathrm{cu}=2=11$ 
ELOM DIA. MANW SUPPLY DRAIN LIS. H. CDEFF SLOPE SLCPF. (SIN) (SIN)
CRAIN FLOH

ENTRY JEPTH

RATIS H/D.
ENTRY

ENERGY

M.
NORMAL DEPTH

M/O.
PIPE LEVGTH NORMAL 'SEPT: L/D.

\section{$2.00 \quad 0.100 \quad 0.7090 \quad 0.2588 \quad 0.0167$ \\ $4.00 \quad 0.100 \quad 0.0210 \quad 0.25 \varepsilon 6 \quad$ r. ilb7 \\ $\begin{array}{lllllll}0.00 & 0.100 & 0.0010 & 0.2566 & 0.0167\end{array}$ \\ $8.00 \cong .100 \quad 0.20700 .2586 \quad 0.0167$ \\ $10.040 .10 C \quad 0.013+0.0568 \quad(.4167$ \\ $12.00 \quad 0.100 \quad 0.0030 \quad 0.258 t \quad$ C.0137}

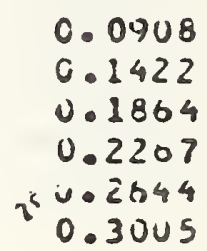

0.0737

0.1148

0.2445

0.1013

0.2200

0.23 .39

0.0660

0.1026

U. 1334

0.1615

0.1874

0.2120

0.0620

$2.00 \quad 0.100 \quad 0.00700 .8660 \quad 0.0167$

$4.00 \quad 0.10 C$ U. 0070 0.E七tC C.CIA7

$6.00 \quad 0.100$ C. $0030 \quad 0.86 \in C$ C.C.167

$8.000 .1004 .00300 . \operatorname{ett}$ C.0.167

$10.00 \quad 0.160 \quad 0.0070 \quad 0 . E 6 t C \quad 0.0167$

$12.00 \quad 0.100 \quad 0.00 \geqslant 0$ 0.8ntC C.U16?
0.0961

0.1249

0.1510

6.1752

0.1479
0.2566

0.4174

0.5467

0.0575

0.7255

0.0425

0.3830

0.6302

0.0355

1.0100

1.1079

1.3094

0.4749

0.7849

1.0441

1.2663

1.4704

1.0537
0.2242

0.3645

0.4917

0.6109

0.7271

0.8403

0.2242

0.3645

0.4 .317

0.0108

ن. 7271

0.84 ن 3

0.2242

0.3645

0.4917

0.6108

0.7271

0.8403
55.3242

80.3375

116.6237

130.5984

155.0110

164.9513

58.5317

94.3193

124.0124

145.7407

165.6441

162. 1040

59.9177

96.6904

1.27 .2047

149.7349

170.4153

107.4975

0.2242

0.3645

0.4917

0.6108

0.7271

0.8403

0.2242

0.3645

0.4917

0.6103

0.7272

0.8403

0.2242

0.3645

0.4917

0.0148

0.7271

0.6403
60.6230

97.9343

125.8941

153.1159

$150.60: 9$

$170.00 \leq 0$

60.3943

$90.50=3$

126.9101

$15 c .8503$

$14<.6712$

$17<.9530$
61.0873

$90.75 \leq 0$

128.0254

157.3017

$13 y \cdot 1301$

171.5073

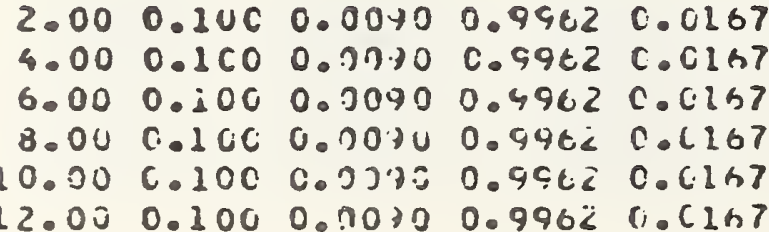

0.0593

0.0719

0.1143

0.1442

0.1671

0.1008
0.5658

0.4757

1.3006

1.5839

1. 6415

2.4770 


FLOH DIA. MANH. SUPPLY OFAIN
LIS. M. COEFF SLOPE SLCOE
(SIN) (SIN)

(SIN) (SIN)
DRAIN FLOH

ENTRY UEPTH

RATIO H/U.
EATRY

ENERGY

M.
NURMAL OEPTH

H/O.
PIPE LENGTH T NORMAL UEPTH. L/O.

\begin{abstract}
$2.00 \quad 0.100 \quad 0.0070 \quad 0.2580 \quad 0.0125$
$4.00 \quad 0.1 C C \quad 0.77100 .2560 \quad 0.0125$

$6.00 \quad 0.100 \quad 0.1070 \quad 0.25$ t 0.0125

$8.00 \quad 0.1(0) 0 . n 3 t 1) n .2550 \quad 0.0125$

$10.00 \quad 0.1000 .30) 00.2 \leq 5 \mathrm{C} \quad 0.0125$

$12.060 .100 \quad 0.3270 \quad 0.2560$ C.C125
\end{abstract}

0.0948

0.1422

0.1804

$u .2267$

C. 2644

C. 3005

0.0737

0.1144

0.1445

0.1313

$0.210 r$

0.2304

c. 0600

U. 1020

u. 1334

0.1613

U. 1874

0.2120

0.0620

i. 0701

6. 1244

0.1510

0.1722

U. 197

$0.05+0$

0.092 t

u. 1245

. .1450

v. lusc

U.1 1us

i. $05 y 3$

i. $091 \%$

ن. 11 's

ن. 1442

ᄂ. 1671

0.1200
0.2566

0.4174

0.2467

0.0275

0.7555

0.0423

\subsection{0}

0.0302

0.0355

1.0108

1.1079

1.3499

0.4749

0.7847

1.0441

1.2565

$1.47 \mathrm{C4}$

1.5537

0.5374

0.5921

1. 1491

1.4457

$1.07 \leq 5$

$1.0 y 42$

0.2752

0.4555

$1.274 j$

$1 .=226$

1. 0152

2. $43>2$

$0.5 t 58$

ग. $\rightarrow 75,7$

1.3000

1.2. 3?

1.0414

2. 477.
0.2478

0.4050

0.5403

0.6941

0.8149

ن. 9439

0.2478

0.4050

0.5433

0.6841

0.8149

0.9430

0.2478

0.4050

0.5403

3.6541

0.0149

U. 9438

0.2478

0.4050

J. 5403

J.5.441

U. 3149

0.9430

0.2478

0.4050

0.5403

J. .6041

0.5149

v. 9435

0.2478

0.4050

0.5403

0.0 .41

0.0149

0.9433
39.4000

94.3054

12 L. 5331

141. EYL 1

$150.98=3$

164.0313

62.6177

97.7113

120.9154

130.4735

107.5933

18 .

64.0026

$10<.0411$

130.7371

152.0251

$170.24,2$

195. 32513

64.6025

$107.107 j$

130.4212

150.5441

109.3000

2uें. $484 \mathrm{~J}$

04.3255

104. $13=4$

$13 v .1414$

$144 .+5 \div 5$

160.0. 0 ?

$200 \cdot v 3 y 3$

64. $2>01$

$11 i .7127$

13ueveso

$141.7 \%$,

leopivel

$207 \cdot 0.3$ 
FLOH DIA. MAWH. SUPPLY OKAIN L/S. M. COEFF SLLPE' SLCPE (SIN) ISIN]
DRAIN FLOH

ENTRY DEPTH

RATIO H/D.
ENTRY

ENERGY

M.
NORMAL DEPTH

HOP.
PIPE LEVETH NORAAL JEPTH L/O.
$2.00 \quad 0.100 \quad 0.0120 \quad 0.2588 \quad 0.0500$

$4.00 \quad 0.100 \quad 0.31 \geq 0 \quad 0.2 \leq E 8 \quad 0.0500$

$6.00 \quad 0.100 \quad 0.1120 \quad 0.25 E$ E 6.6500

$8.00 \quad 0.160 \quad 0.21: 30.2 \pm 80 \quad 0.0500$

$10.00 \quad 0.100 \quad 0.31200 .2566 \quad 0.05001$

$12.00 \quad 0.100 \quad 0.0120 \quad 0.2568 \quad 0.0500$

\author{
0.1092 \\ 0.1722 \\ 0.2267 \\ 1.. 2706 \\ l. 3235 \\ 0.3009
}

\subsection{9}

0.2921

0.3797

0.4540

0.5194

0.57 .62

$2.00 \quad 0.100 \quad 0.21200 .5000 \quad c .0500$

$4.00 \quad 0.1 C 0 \quad 0.3120 \quad 0.5$ COC C. 1.500

$6.000 .1 C$ C. C. $21200.5 C(C)$ C. 5500

$8.00 \quad 0.100 \quad 0.71200 .5 \operatorname{ccc} C .0 .500$

$10.00 \mathrm{C} .100 \quad 0.121200 .2 \mathrm{COO} C .0 \mathrm{C}=50$

$12.000 .1000 .312 \% 0.50000 .6500$

$2.00 \quad 0.1000 .2120 \quad 0.7070 \quad 0.0500$

$4.000 .160 \quad 0.21200 .7070 \quad c .0500$

$6.00 \quad 0.1 C 0 \quad 0.01200 .7 C 7 C \quad 0.4500$

$8.00 \quad 0.100 \quad 0.31200 .7070 \quad$ C.L.500

$10.00 \quad 0.100 \quad 0.01200 .707 C \quad 0.0500$

$12.00 \quad 0.100 \quad 0.3120 \quad 0.7070 \quad 0.0500$.

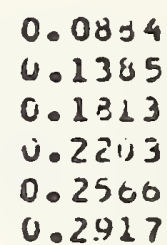

0.0792

0.1237

0.1615

0.1457

C. 2279

0.2585
0.2695
0.4387
0.5765
0.6939
0.7998
0.8915

0.3332

0.5457

0.7196

0.8715

1.0041

$1.123 b$
0.1879

0.3030

0.4050

0.5015

ग. 5842

0.6841

0.1879

0.3030

0.4050

0.5015

0.5442

0.6341

4.1879

0.3030

0.4050

0.5015

0.5742

0.6341
23.6006

36.9355

50.07 .13

61.3337

70.6221

70.2407

$26.24 \times 4$

43.3731

56.5107

68.3543

78.3214

67.3712

27. 3نे०y

49.1449

50.6053

$71.31<1$

$8 \mathrm{z} \cdot 17 \mathrm{io}$

$91.1 \%$ 1

0.1879

0.3030

0.4050

0.5015

0.5442

0.6941

0.1879

0.3030

0.4050

0.5015

0.5942

0.6842

1.3062

0.4117

0.0771

0.0973

1.0894

1.2000

1.4150
0.1879

0.3030

0.4050

0.5015

0.5942

i. bo 41
27.8405

46.0473

60.1240

$76.82 \cup 1$

83.9443

93.2107

20.1133

40.2043

60.7327

73.5814

84.2113

$94.211 j$

20.1907

40.6272

64. 3042

73.7700

$82.003 \mathrm{~J}$

$94.40 \times 1$ 


FLOH OIA. MANN SUFPLY OFAIN
L/S. .H. COEFF SLOFE SLIPE
ISINI ISINI

$$
2.00
$$

$2.00 \quad 0.100 \quad 0.01200 .5000 \quad 0.6 .167$

$4.00 \quad 0.100 \quad 0.31200 .5 \operatorname{ccc} \quad 0.0167$

6.Cu C.1C0 0.112U J.EC.C.U C.Clo

9.0 $0.1 C C$ C.1120 0.ECOC C.LIh]

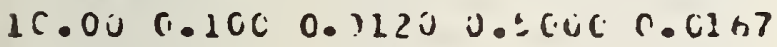

$12.000 .100 \quad 0.31 \geq 00.5 C C L(.4157$
DEAIH FLCH

FNTKY DEPTM

RATIO H/D.
ENTRY

ENERGY

n.
N:IRMAL DEPTH

H/O.
PIPL LEATTH TO NORTIL JEPTH. L/O.

$\begin{array}{llll}0.1092 & 0.1019 & 0.2737 & 34.0436 \\ ن .1722 & 0.2421 & 0.4500 & 50.8530 \\ 1.2207 & 0.3797 & 0.6100 & 0.3500 \\ u .2756 & 0.4540 & 0.7051 & 72.5120 \\ 0.3235 & 0.5194 & 0.9146 & 75.51100 \\ ن .3004 & 0.3762 & 1.0000 & 70.5504\end{array}$

0.0064

0.1305

0. 1313

0.2203

6. 2566

0.2917

i. 0742

U. 1237

6.10is

ن. 1927

i. 227 's

U. 25.55

0.0743

o. 1123

ن. 1510

0.1 .27

$0.2: 25$

v. 2408

$0.07 i 7$

i.111

0.1450

v. 1701

4.2050

v. $23<1$

0.0710

U. 1107

0.1442

0.1744

‥ $2 \cdot 3<=$

$0.22 \div 0$
0.2695

0.4387

0.5765

0.6439

$0.7 \times 98$

0.0912

0.1332

0.2457

0.7193

0.0715

1.0041

1.1235
0.2737

0.4500

U. 61 U

J.7651

0.9146

1.0000

0.2737

$0.45 \cup 0$

0.61 is 8

0.7621

0.9146

i. 0000

0.3769

0.5199

0.0140

0.7951

1. 1447

1. 2094

0.4030

0.0541

0.0797

1.0034

1.<3 3:

2. suth
0.2737

$0.45 \cup 0$

$0.01 \cup 8$

0.7001

.0 .1140

1. 0500

0.2737

0.4500

$0.61 \cup 8$

U. $76>1$

J. 7146

i. Duu ú
$32.027 \mathrm{~s}$

54.0024

00.3731

70.5076

82.3177

$70.91 i$ J

$32.97>5$

50.2017

74.6235

$84.47) 3$

77.057 J

82.731\%

36.5011

$57.1+y$ क

72.3390

Y4. 4325

24.2125

04.3231

30.7047

50.2235

73.0122

$74.0: 43$

ว. 2132

os. 2910

30.5111

$0.4117 \quad 0.2737$

$0.6 .771 \quad 0.4500$

$3 c .7422$

0.3473

$0.01 \cup 0$

$0.70>1$

1.0044

0.9146

1.4250

L. Uuvo

74.2127

$74 .+107$

- . 7203

oc.irsil 
FLOM DIA. MAYN. SUPPLT CRAIN L/S. H. COEFF, SLOPE SLOPE (SIN) (SIN)
DPAIN FLOH ENTRY ENTRY DEPTH ENERGY RATIO HSD. n.
NORMA L DEPIH Jifo.
PIPE LEVGTH I NORMAL JEPTH. L/D.

\begin{abstract}
$2.00 \backsim 100 \quad 0.0120 \quad 0.2568 \quad 0.0250$
$4.00 \quad 0.100 \quad 0.1) 120 \quad 0.258 \varepsilon \quad 0 .(250$

$6.00 \quad 0.100 \quad c .3120 \quad 0.25880 .0250$

$8.00 \quad 0.100 \quad 0.21200 .258 \varepsilon \quad 0.0250$

$10.000 .100 \quad 0.21200 .25 t 60.1250$

$12.00 \quad 0.100 \quad 0.3120 \quad 0.25880 .0250$
\end{abstract}

$2.00 \quad 0.100 \quad 0.3120 \quad 0.50000 .0250$

$4.00 \quad 0.100 \quad 0.0120 \quad 0.500 C \quad 0.0 .55$

$6.00 \quad 0.100 \quad 0.01200 .5000 \quad 0.0250$

$8.000 .1600 .3120 \quad 0.5 C(C) \quad 0.0250$

$10.00 \quad 0.1000 .31200 .5 C C C$ C.C.5 50

$12.00 \quad 0.100 \quad 0.31 \geq 0 \quad 0.5000 \quad 0.4250$

$2.00 \quad 0.10 C \quad 0.3220 \quad 0.7076 \quad 0.0250$

$4 . C 00.1000 .31200 .7 C 70 \quad 0.0250$

$6.00 \quad 0.100 \quad 0.91200 .7570 \quad$ C.C250

$8.00 \quad 0.160 \quad 0.21 \geq 0 \quad 0.7070 \quad 0.6250$

$10.00 \quad 0.160 \quad 0.9120 \quad 0.7070 \quad 0.1250$

$12.00 \quad 0.1000 .31230 .7070 \quad 0.0250$

$2.00 \quad 0.1 C 0 \quad 0.01200 .86 t 0 \quad 0.0250$

$4.00 \quad 0.160 \quad 0.11120 \quad 0 . E 6 t 0 \quad C . C 250$

$6.00 \quad 0.160 \quad 0.0120 \quad 0.96 t U \quad 0.0250$

$8.00 \mathrm{C.LCC} 0.32200 .8 \mathrm{0.20}: \mathrm{C.U250}$

$10.00 \quad 0.10 \mathrm{C} \quad 0.31200 .5660 \quad 0.0250$

$12.00 \quad 0.100 \quad 0.31200 .8660 \quad(.0250$

$2.00 \quad 0.100 \quad 0.31200 .46590 .0250$

$4.00 \quad 0.100 \quad 0.3120 \quad 0.96590 .0250$

$6.00 \quad 0.100 \quad 0.01200 .9459 \quad .1250$

$8.00 \quad c .100 \quad 0.01200 .9 t 59 \quad 0.0250$

$10.00 \quad c .100 \quad 0.01200 .9659$ C.C.250

$12.00 \quad 0.100 \quad 0.3120 \quad 0.9659 \quad 0.0250$

\begin{abstract}
0.1092
0.1722

0.2267

0.2756

0.3235

0.3609
\end{abstract}

0.0804

0.1365

0.1813

0.2203

0.2566

0.2827
0.1819

0.2921

0.3747

0.4540

0.5144

0.5762

0.2095

0.4387

0.5765

0.6937

0.7978

0.6915

0.0742

0.1237

0.1015

0.1937

0.2279

0.2535

0.0743

0.1150

0.1510

0.1327

0.2125

0.2408

$0.0 ? 17$

0.1118

0.1456

0.1701

4.2050

0.2321

0.0710

c. 11307

0.1442

0.1744

0.2028

U. 2210
0.3332

0.5457

0.7196

0.0715

1.0041

1.2235

0.3769

0.6197

0.8193

0.7951

2.2497

1.2094

0.4036

0.0041

0.07797

1.0684

1.2333

1. 3862

0.4117

0.0973

1. 0804

1. $<$ OU

1.4150
0.0771
0.2377

0.3879

0.5239

0.6528

0.7778

0.8999

0.2377

0.3879

0.5239

0.0528

0.7778

ט. 8499

0.2377

0.3879

0.5239

0.6528

j. 7778

0.8979

.0 .2377

0.3979

0.5239

0.6528

0.7778

0.3999

0.2377

0.3879

0.5239

0.0528

0.7778

0.8999

0.2377

0.3374

0.5239

$0.55 \angle 6$

0.7774

$0.09 y 9$
30.2759

48.5540

$02.44 \cup 5$

$73.10 v 0$

81.9357

80.7532

32.5305

52.3407

67.5335

74.4037

64.16 .99

90.3447

35.5040

54.0006

09.7640

$6<.1042$

$9<.3702$

100.5153

34.0100

54.9775

70.4434

83.6923

94.1377

102.5057

$34.27+3$

$5=.3210$

71.5533

84.4010

94.1475

103.6423

34.3242

55.4413

71.7130

64०.13 44

95.2473

109.37 .77 
CIA.

m.

MANT.

C.JEFF
SUPPLY CFAIN

SLI:PE SLLPF

(SIN) (SIV)
ORAIN FLCH ENTRY ENTRY OEPTM ENERGY RATIO H/C.
NORMAL DEPTH H/O.
PIPE LENGTH TL NORALL JEPTH. L/U.
$2.00 \quad 0.100 \quad 0.01 \geq 0 \quad 0.2586 \quad 0.0125$ $4.00 \quad 0.100 \quad 0.3120$ 0.Z.EE (..C1.5 $6.00 \quad .100 \quad 0.31200 .25 t c \quad(1.0223$

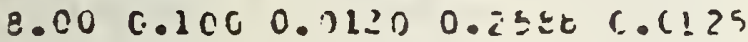
$10.000 .160 \quad 0.11 \geq 0 \quad 0.25=6 \quad 0.1 .12 \div$ $12.00 \quad 0.1600 .31200 .2588$ C.LL?

$2.000 .14 C 0.01200 .50 C C \quad c .0125$ $4.1 .50 .1 C C \quad 0.3220 \quad 0.5 C C C \quad 0.0225$ $6.16 \quad 6.200 \quad 0.121200 .5 \operatorname{ccc}(1.0 .125$

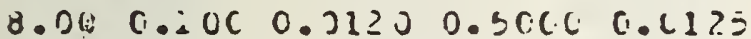
$10.00 \quad 0.16 .0 \quad 0.01200 .5 C C U$ C.Cl25 $12.00(.1000 .121200 .500 \mathrm{cov}(.51 ? 5$

$2.00 \quad 0.100 \quad 0.01230 .7070 \quad 0.01 \% 5$ $4.0 v 0.100,1.11200 .7 C 7 C \quad 6.0125$ $0.000 .100:-1200.7070$ C.0.:2 $8.00 \quad 0.100 \quad 0.0120 \quad c .7070 \quad c .4125$ $10.00 \mathrm{C.IUC} 0.31200 .707 \mathrm{C}$ c.0125 $12.00 \quad 0.1 \cup 0 \quad 0.91200 .7670 .0125$

2.CG 0.1UC 0.01200. Etell 0.0125

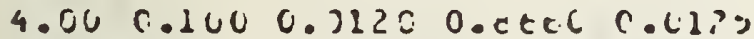
$6.00 \quad 0.100 \quad 0.3220 \quad 0 . t . t+0 \quad 0.0125$ $8.00 \mathrm{C.1CC} 0.31100$ D.feiv C.C18=

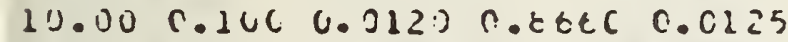
$12.00 \quad 0.10 \mathrm{C} 0.31 \geq 0 \quad 0.86 t u$ C.U. 125

$2.00 \operatorname{col} 100 \quad 0.0120 \quad 0.9659 \quad 0.012=$ 4.F: $0.1(10$ c. $31 \geq 0.4 E 590.0: 25$

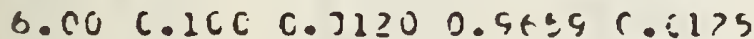
$\because .00 \mathrm{C.1CC} 0.01200 .46 .54 \quad 0.5 .125$ $10.00 \mathrm{C} .10 \mathrm{C} 0.3120 \mathrm{~J} 0.96 \leq 4 \quad \mathrm{Cu} 22$ $12.00 \quad 0.1600 .11200 .5054 \quad 0.0133$

2.00 C.JCO $0.31230 .996: C . C_{2} \geq 3$ 4.0C C.1CC 0.)1:j 0.4>is (.U1:3

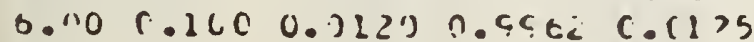
b. (u) 0.1ur. C. .11?? 0.44t e r.el1?:

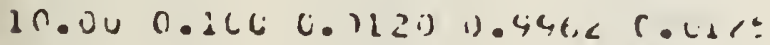
$12.000 .100 \quad 0.31200 .9742 \quad C .(1) 5$

\begin{abstract}
0.1092
0.1722

0.2267

4.2766

U. 3232

0.3689
\end{abstract}

0.0384

C. 1345

U. 1313

C. 2203

C. 2506

c. 2417

0.0792

C. 1237

c. 1615

$0.19=7$

ט. 2274

v. 2505

$0.0: 43$

C.1150

0.15 iv

$0.1=27$

ن. 2125

0.2408

ט. 0717

c. 1116

U. 1453

$0.170 i$

(. $23>0$

0.2321

i. 0710

C. 11.07

U. 144 ?

c. 1744

‥ 2.32

अ. 22,0
0.1819

0.2121

0.3797

0.4540

0.3194

0.3702

0.2695

0.4387

0.3765

0.0937

0.7995

0.4915 .

0.3332

0.5457

0.7196

0.0715

$1.004 i$

1.1239

0.3769

0.019 .1

0.0193

0.4951

1. 2447

1.2694

0.4030

0.0041

C. .5797

1.0644

1.4330

1.3862

0.4117

0.0771

0.5773

1. 4094

1.200J

1.4150
0.3030

0.5015

0.6841

0.5509

- 1. vuบo

1. 0ũúo

ن. 030

0.5015

0.0841

j. 8509

1.0000

$1.00 \cup 0$

0.3030

0.5015

0.6541

$0.85=9$

1. 0000

1. 0 ن vu

0.3030

v. 5025

0.6441

0.0509

1. $0: 200$

1.0000

0.3030

ن. 2015

J.3.54

J. 35.99

1.1) Duo

1. 0uvo

0.3030

0.5013

0.0 .341

נ. $20=4$

1. 0000

1. $0: 10$
$3<.6271$

44.001

50.81149

64.1542

$05.5>04$

59.3663

34.8002

$5<.7402$

$03.37,3$

70.4113

$7<.3>24$

67.5720

35.7892

53.6027

C2.5029

$72.40 ; 3$

75.h>l

7 i. 3477

30.0054

> 4.51 i

00.4357

74.3143

77.4503

73.4072

30.1100

51.4450

$00 . \div 327$

7:-10ua

$70 \cdot 37: 1$

74.44 .7

30.217.

$24.7 \div 11$

ce.443j

$72.1: 3$

70.116 .4

74.73 .16 
FLOH CIA. MANN. SUFPLY CRAIM L/S. H. CUSFF SLOPE SLDPE (SIN) (SIM)
DRAIN FLOH ENTRY DEPTH RATIO H/O.

ENTRY
ENERGY
H.

H.
NORMAL

DEPTH

:H/D.
PIPE LENGTH MORAAL DEPTH L/O.

\begin{abstract}
$2.00 \quad 0.20 C \quad 0.0150 \quad 0.2588 \quad .0500$
$4.00 \quad 0.100 \quad 0.1150 \quad 0.2580 \quad 0.05 n 0$

$6.00 \quad 0.140 \quad$ C. 31500.2568 C.C500

$8.00 \quad 0.100 \quad 0.31500 .25 \varepsilon 6 \quad$ C.LEnO

$10.00 \quad 0.10 C \quad 0.02500 .25 E E$ C.CSOn

$12.000 .100 \quad 0.01500 .25400 .4500$
\end{abstract}

$2.00 \quad 0.100 \quad 0.015000 .5000 \quad 0.0500$

$4.000 .10 C \quad 0.91500 .5000 \quad 0.0500$

$6.0 C \quad 0.1 C C \quad 0.01500 .5 C C C$ C.C530

$8.00 \quad 0.1 C 0 \quad 0.7150 \quad 0.50 C$ C. 0.000

$10.00 \quad 0.106 \quad 0.31500 .58000 .050$

$12.000 .1600 .32 j 00.50000 .0500$

$2.00 \quad 0.1 C C \quad 0.01500 .7 C 7 C \quad 0.0500$

$4.000 .1 C 0.31500 .7070$ c.0.500

$6.00 \quad 0.100 \quad 0.0150 \quad 0.7 C 7 C \quad 0.0500$

$8.00 \mathrm{C.10C} 0.11500 .7070$ C. 050 no

$10.00 \quad 0.10 C \quad 0.0150 \quad 0.70 .70 \quad$ C.C500

$12.00 \quad 0.100 \quad 0.7150 \quad 0.70 .700 .01500$

$2.00 \quad 0.100 \quad 0.3150 \quad 0.8000 \quad 0.0500$

$4.00 \mathrm{C} .1 \cup \mathrm{C} 0.3150 \quad 0 . t \in 60$ C.C.500

$6.00 \quad 0.10 C \quad 0.02500 .60 C 0$ C. 0.500

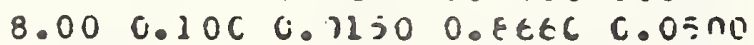

$10.00 \quad 0.100 \quad 0.3150 \quad 0.6640 \quad 0.0503$

$12.00 \quad 0.100 \quad 0.3150 \quad 0.8660 \quad 0.0500$

$2.00 \quad 0.100 \quad 0.0150 \quad 0.9659 \quad 0.0500$

4.000 .1 CC L. $.31500 .5 t=9 \quad 0.6590$

$6.00 \mathrm{C.1CC} \quad 0.0150 \mathrm{C.SC} \leq 90.0500$

$8.00 \mathrm{C.1CC} 0.31500 .9654$ C.U500

$10.00 \quad 0.10 \mathrm{C} \quad 0.02500 .4659$ C.c500

$12.00 \quad 0.1 C C \quad 0.01530 .9459$ C.CSOH

$2.00 \quad 0.160 \quad 0.0150 \quad 0.99620 .0500$

$4.00 \quad 0.100 \quad 0.01500 .9962 \quad 0.1450$

$0.00 \mathrm{C.ICC} \mathrm{0.0150} \mathrm{0.59Ez} \mathrm{C.0.500}$

$5.000 .1<0 \quad 0.12>0 \quad 0.99 t 20.15 n 0$

$10.00 \quad 0.100 \quad 0.3150 \quad 0.944 \bar{c} 0$. isno

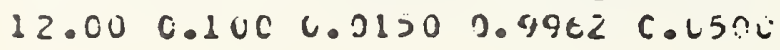

\subsection{3 \\ 0.2001 \\ 0.2644 \\ 0.3235 \\ 0.3901 \\ 4.934}

0.1020

0.1605

0.2108

0.2566

0.3040

0.3420

0.0912

0.1432

0.1874

0.2279

0.2659

0.3020

0.0855

0.1339

0.1752

0.2125

0.2470

0.2515

0.0926

0.1243

0.1658

0.2050

$0.230 t$

0.2700

$0.0 y 18$

0.1278

0.1671

0.2028

0.2302

0.2370
0.1404

0.2237

0.2089

0.3441

0.3907

0.4325

0.2062

0.3325

0.4339

0.5211

0.2961

0.0616

0.2540

0.4121

0.5413

0.6509

0.7476

0.8349

0.2374

0.4622

0.6155

0.7435

0.8546

0.9544

0.3072

0.5009

0.0607

$0.797 \mathrm{v}$

0.7189

1.0283

0.3125

0.5120

0.0737

0.0137

0.9371

1.0500
0.2184

0.3552

0.4778

0.5942

0.7056

0.8149.

0.2184

0.3552

0.4778

0.5442

$0.70>6$

0.8149

0.2154

0.3552

0.4778

0.5942

0.7056

0.8149

0.2284

0.35 .2

0.4770

0.5442

0.7036

0.8149

0.2104

0.3552

0.4778

0.5942

0.7056

0.3149

0.2184

0.3552

0.4778

0.5942

0.7056

0.6149
10.6500

27.3550

35.2204

42.0155

40.7747

51.1932

16.5129

30.3949

39.2359

40.3632

$5<.3797$

57.4265

$19.25=31$

31.5737

40.41253

40.9370

$54 .=147$

60.2075

19.6037

32.3404

41.8513

50.0500

50.1120

61.6505

19.8852

$32.07+7$

$4<.1201$

50.397,

$5 c .7715$

6<. 4205

14.9352

32.7547

42.4444

$50.75=2$

50.1940

6टे००८>5 


LLOH DIA. MINN.
HS. H. CDEFF

SUPPLY CPAIN

SLCPE SLCPE

(SIN) (SIN)
ERA IN FLOH

ENTRY OEPTH ENTER

RATIO H/O. . T.
NORMAL - DEPTH H/D.
PIPE LENUTA TO MORMIL UEPTH. L/D.

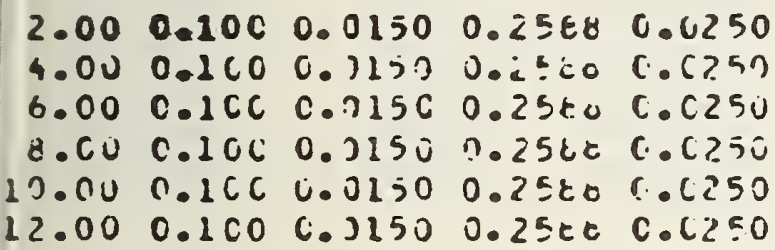

$2.00 \quad 0.1 C 0 \quad 0.1150 \quad 0.5 C C C \quad 0.0250$

$4.000 .16 C 0.31500 .50 C C$ C. 0250

$0.00 \quad 0.1 C 0 \quad 0.1150 \quad 0.50 L C 0.0250$

$8.00 \quad 0.16(0)$.100 C.scec $0.0 ? 50$

$\left.10.00 \quad C .10 C \quad U_{0}\right) 150 \quad 0.2 C E C$ C. 2250

$12.00 \mathrm{C.ICO} C .150 \mathrm{j.sOCu}$ C. 125.$)$
0.1263

0.20121

0.2644

0.3235

0.3501

0.4343

0.1020

0.1605

$0.214=$

0.2506

ن. 3.500

0.3420

0.0712

0.1432

U. 1374

U. 2279

C. 2559

0.3020

0.0355

C. .1339

0.1752

0.2125

$0.247 \pm$

0.2325

0.0320

0.1273

0.1608

0.2050

U. 2336

ن. 27.8

$0.0 \leq 18$

$0.127=$

0.1071

U. $20.2=$

$0.236 c^{\circ}$

0.2070
0.1404

0.2237

0.2059

0.1441

0.3907

0.4325

0.2062

0.3325

0.4339

0.2211

0.5961

0.0516

0.2540

0.4122

0.5413

$0.630 \mathrm{r}$

0.7476

0.8349

$0: 2874$

0.4682

0.0155

0.7435

0.0540

0.9544

0.3072

0.2009

0.0607

0.7470

0.3133

1. 0243

0.3125

0.3120

0.0737

0. 0137

0.1371

1. USO'J
0.2776

0.4573

0.0216

0.7778

0.9302

1.0000

0.2776

0.4573

0.0210

0.7718

ט.93uz

1. 0000

0.2776

0.4573

0.6216

0.7770

0.9342

1.0000

0.2776

0.4573

0.6216

0.777 y

0.93 .12

2. 0000

0.2776

0.4573

0.6216

0.7778

0.7302

1. Uuvo

0.2776

0.4373

0.0216

$0.777 \mathrm{c}$

$0.43 \cup 2$

1. OUUC
19.8050

30.7053

$30.03+2$

$4<.7503$

43.1730

39.4003

21.4307

33.4122

$41.665 i$

$47.28=0$

$51 \cdot 020$

45.3241

$22.15>0$

34.6347

$43.33 \div 2$

49.2103

53.4375

4 c. 1274

$2<.54<2$

35.3003

44.2111

Su. $3 \bar{c}^{3} 0$

$54.71=1$

44.0075

$2<\cdot 7340$

3). 5314

$44.57<4$

30. $3=13$

52.40 .57

$50 . j+2 ?$

$2<.70=1$

35.7351

44.7 .23

51.0423

53.0733

5u. 1.:2:4 


FLOW OIA. MAHN. SUPPLY ORAIN
LIS. H. COEFF SLOPE SLCPE

(SIN) ISINI

ORAIN FLOH ENTRY DEPTH RATIO HID.

\section{EPTRY \\ ENERCY \\ M.}

MORMAL
DEPTH
H/O.
PIPE LEITGTH NORAAL UEPTH L/D.

\subsection{3 \\ 0.2001 \\ 0.2644 \\ 0.3235 \\ 0.3301 \\ 4.4343}

0.1020

0.1505

0.2100

0.2560

0.3000

0.3420

0.0912

0.1432

0.1074

0.2279

0.2654

0.3020

0.0355

0.1339

0.1752

0.2125

0.2478

0.2615

0.0326

0.1273

0.1650

0.2050

0.2350

$0.270=$
0.1404

0.2237

0.2889

0.3441

0.3907

0.432 .5

0.2062

0.3325

0.4339

0.5211

0.5901

0.0616

0.2540

0.4121

0.5413

0.0505

0.7476

0.3349

0.2874

$0.46 t 2$

0.3155

0.7435

0.0948

$0 . \rightarrow 544$

0.3072

0.5009

0.0007

0.7970

0.4148

1.0283

0.0318

0.1274

0.1671

0.2320

0.2302

0.2578
0.3125

0.3120

0.0737

0.8137

0.4371

1. 02013
0.3201

0.5317

0.7271

$0.9: 80$

2.0000

1.0000

0.3201

0.5317

0.7271

0.9146

1. 0000

$1.00 \cup 0$

0.3201

0.5317

0.7271

0.9146

1.0000

1.0000

0.3201

0.5317

0.7271

0.9146

1.0000

1.0000

0.3201

0.5317

0.7271

0.9146

$1.00 \cup 0$

1.0000

0.3201

0.5317

0.7271

0.9140

1.0000

1. 0000
16.9707 27.4700 31.2717 31.3953 34.2745 32.0278

20.5906 $30.1=02$ 34.4144 34.7729 34.3445 37.7511

21.3256 31.4352 30.0021 35.7424 41.7854 40.4453

21.7209 $3<017 y 7$ 37.5057 35.7791 43.0414 4 i. 9543

21.9229 32.3039 37.1301 35.4011 43.7029 42.7323

$21.97 j 3$ 33.0017 $30.10 \times 2$ 30.3144 43.1433 46.4402 


FLOK DIA. MANN. SUPPLT CRAIN
L/S. H. COEFF SLOPE SLCPE
(SIN)
(SIN)

$2.00 \quad 0.100 \quad 0.3130 \quad 0.2586 \quad .05 n 0$

$4.000 .1000 .21300 .2560 \quad 0.0500$

$6.00 \quad 0.100 \quad 0.02160 .25600 .0500$

$8.00 \quad 0.100 \quad 0.1110 \quad 0.2558 \quad 0.0500$

$10.00 \quad 0.100 \quad 0.01300 .25 e c 0.6500$

$12.000 .8000 .3130 \quad 0.25800 .0500$

$2.00 \quad 0.10 C \quad 0.0130 \quad 0.500 C \quad 0.0500$

$4.00 \quad 0.10 C \quad 0.0190 \quad 0.5000$ C. 6500

$6.00 \quad 0.100 \quad 0.31300 .5000 \quad 0.1300$

$8.00 \mathrm{C.10C} 0.01300 .5 C \mathrm{Cu}$ C.L.500

$10.00 \quad 0.1000 .02300 .5 \operatorname{CCC} C .0 .500$

$12.00 \mathrm{c} .1000 .13300 .5000 \quad 6.0500$

$2.00 \quad 0.100 \quad 0.01300 .70700 .0500$

4.00 C.ICC 0.31900 .7070 C.L500

$6.00 \quad 0.100 \quad 0.0130 \quad 0.7670 \quad 0.0500$

$8.00 \quad 0.100 \quad 0.21300 .7670 \quad 0.6500$

$10.00 \quad 0.100 \quad 0.3150 \quad 0.70700 .6500$

$12.00 \quad 0.100 \quad 0.3100 \quad 0.7070 \quad 0.0 .500$

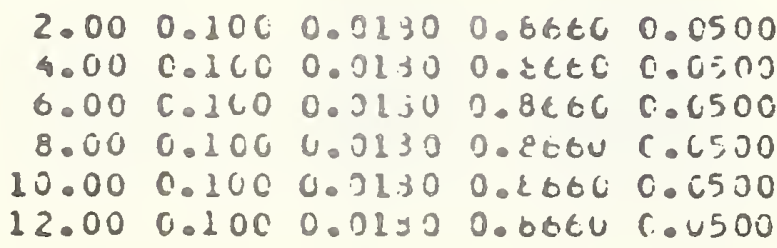

$2.00 \quad 0.100 \quad 0.01300 .5659$ C. 5500

$4.00 \quad 0.16 C \quad 0.133+0.5 .9654$ C.C5n0

$6.00 \quad 0.200 \quad 0.31300 .0 .5 .9$ C.c.sno

$8.000 .160 \quad 0.91300 .96540 .6500$

$10.00 \quad 0.100 \quad 0.31 .10 \quad 0.9 t \leqslant 4 \quad$ C. 0.30

$12.00 \quad 0.2000 .31300 .4659$ C.C500

$2.00 \quad 0.100 \quad 0.0190 \quad 0.9962 \quad 0.0500$

$4.00 \quad 0.1000 .01330 .992<\quad 0.6500$

$6.00 \quad C .1 C C$ C.DIJ0 0.49tz C. 0500

$8.000 .1 C 0 \quad 0.01300 .99 t i$ C.C500

$10.00 \quad 0.160 \quad 0.31330 .9462 \quad c .65 n 0$

$12.000 .1000 .31300 .492 \bar{c}$ r.L\$00
ORAIN FLDM

ENTAY DEP.TH

RATIO H/D.
ENIRY

ต.
MORMAL
UEPTH
HOP.
PIPE LENGTH

NOKMAL JEPT

$L / D$.
0.2422
0.2207
0.3005
0.3079
0.4343
0.4976

0.1145

0.1313

0.2308

ن. 2917

0.3420

0.3849

0.1026

0.1615

0.2120

0.2565

0.3520

0.3440

0.0961

0.1520

0.1979

$0.24 j \mathrm{~d}$

0.2315

0.3241

$0.09<2$

0.1450

ง. 19 Ü

0.2321

$0.270=$

0.3079

0.0919

0.1442

0.1008

0.2290

0.2675

0.3044
0.1150

0.1814

0.2332

0.2766

0.3136

0.3462

0.1662

0.2063

0.3454

0.4124

0.4694

0.3218

0.2039

0.3288

0.4243

0.5138

0.5890

0.0546

0.2302

0.3728

0.4884

$0.5<64$

$0.07: 4$

0.7484

$0 . \angle 45$.

0.3991

0.5231

0.0290

0.7224

0.5052

0.2305

0.4068

0.9334

0.0417

0.7374

0.5223
0.2478

0.4050

0.5453

0.6841

0.8149

0.9438

0.2470

0.4050

0.5433

0.6841

0.8144

0.9438

0.2478

0.4050

0.5483

0.6841

0.8149

0.9438

0.2478

0.4050

0.5403

0.6842

0.8149

0.9430

0. 2478

$0.40>0$

0.5453

0.0841

0.8149

0.9439

0.2478

$0.40>0$

0.5483

0.6842

0.8149

0.9430
12.3850

14.4377

24.9284

20.0658

$3 i .5926$

$33.883 \rightarrow$

13.7195

21.6457

$27.51+4$

32.3343

32.5603

30.3230

$14.30>1$

22.3220

$24 \cdot: 253$

33.4132

37.4319

40.4007

14.6122

23.1413

24.

34.7701

$30.41>0$

$41.52 j 2$

14.7077

23.4073

3 U.:.2.

33.2075

30.1300

$4 \therefore .10=y$

14.2130 23.4004 30.2007 35.3230 $34.57+5$ $42 \cdot 27.10$ ? 
FLOH

15
OIA.

MANN.

Ci] TFF
SUPFLY CFAIV

SLOFE SLCPE

(SIN) (SIN)
DRAIN FLUK

EHTRY UEPTH

RAIIJ H/C.
ENT RY

ENERGY

M.
MORMAL DEPTH

$M / D$.
PIPE LENGTH TI NORMAL JEPTH. L/O.

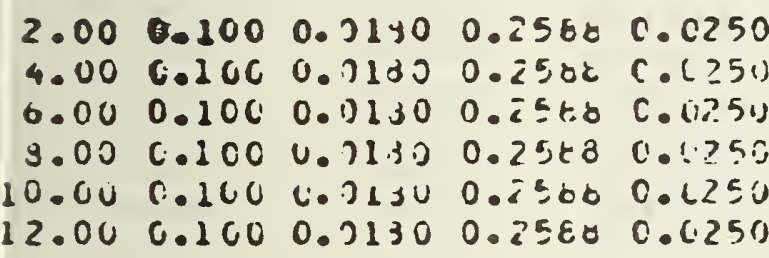

$2.00 C .100 \quad 0.01300 .5 C c c \quad 0.0250$

$4.000 .1000 .31350 .50 C C$ C. 0250

$6.00 \mathrm{C.100}$ C.C15. $0.50 C 0 \quad 0.0250$

8.0. C.1 C C. C!I') U.5CC C.1.? $\$ 0$

$10.00 \quad 0.100 \quad c .11300 .5 c 00 \quad 0.0250$

$12.00 \quad 6.1000 .11300 .50(C$ C.0.250

2.00 C.ICC $0.21300 .7 C 700.0250$

4.Cu r.1CC C.)!3) $0.7(70 \quad 0.1251)$

$6.00 \quad 0.100 \quad 6.31300 .707 C \quad 0.0 .250$

$8.00(1.1600 .310 ? 0.7070 \quad 0.6250$

$13.00 \mathrm{C.1CO}$ C.0130 0.7C.JC C.0?50

$12.00 \mathrm{C.1CO} 4.11100 .7670 \quad 6.4 .250$

$2.00 \quad 0.10 C \quad 0.01150$ n.tete C.0250

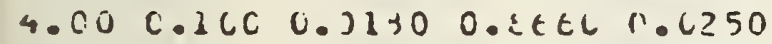

$0.00(.100 \quad 0.21>0 \quad 0 . \varepsilon \in t 0 \quad 0.0251)$

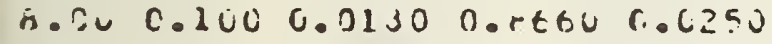

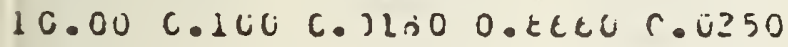

$12.00 \quad 0.1<0 \quad 0.11300 . \varepsilon \in t 0 \quad 0.4 \geq 50$

i:

12.00

$$
\begin{aligned}
& 4.0 \\
& 6.0 \\
& 5.0 \\
& 10.0 \\
& 12.0
\end{aligned}
$$$$
\text { b.00 C.10C } 0.3130 \text { 0.44k2 r.l? >11 }
$$$$
\text { 8.0u c.100 0.3110 0.4ces r.l?50 }
$$

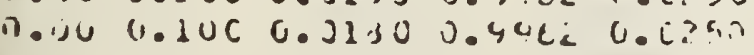

$$
\begin{array}{r}
0.1422 \\
0.2207 \\
6.3005 \\
0.3609 \\
0.4343 \\
0.4976
\end{array}
$$

0.1148

0.1813

0.2359

0.2717

(. $34<$ ()

$0.3>19$

$$
\begin{aligned}
& 0.1026 \\
& 0.1319 \\
& 0.2120 \\
& 0.2505 \\
& 0.30<0 \\
& 0.3440
\end{aligned}
$$

C. 0961

ن. 1510

0.1974

0.2400

$\checkmark .201 E$

v. 32is 1

$0.092 y$

0.1450

C. i9u

0.232i

0.2700

ט. 3074

c. 0419

c. 1442

L. 1306

ن. 2240

c. 257 .

c. 30,44

\subsection{0 \\ $0.1=14$ \\ 0.2332 \\ 0.2766 \\ 0.3135 \\ 0.3462}

0.1062

0.2663

0.5454

0.4124

0.4694

0.bile

0.2039

0.3224

0.4293

0.5139

0.3840

0.6540

0.2302

0.3723

$0.4 \varepsilon 84$

0.3604

0.0714

0.7494

0.2453

0.1991

0.2231

0.0240

$0.7<2.4$

0.0052

0.4136

0.5334

0.0411 
FLOH DIA. MAVN. SUPPLY OKAIH LIS. H. CDEFF SLCPE SLLPE (SIN) (SAN)
DRAIN FLOH ENTRY DEPTH RATIO H/D.
ENTRY

ENERGY

H.
NORMA L

DEPTH

H/O.
PIPE LENITH MOKAAL JEPTH L/D.

\begin{abstract}
$2.00 \quad 0.100 \quad 0.0230 \quad 0.25680 .0167$
$4.00 \quad 0.106 \quad 0.1230 \quad 0.25860 .0157$

$6.00 \quad 0.100 \quad 0.31300 .2566 \quad 0.0167$

$8.00 \quad 0.100 \quad 0.21300 .25840 .0167$

$10.00 \quad 0.10 C \quad 0.31300 .2580 \quad$ C.C.357

$12.00 \quad 0.100 \quad 0.0130 \quad 0.2566 \quad 0.0137$
\end{abstract}

$2.00 \quad 0.100 \quad 0.3280 \quad 0.5000 \quad 0.0167$

$4.00 \quad 0.1 C C \quad 0.2130 \quad 0.5 C O C \quad 0.0137$

$6.00 \mathrm{C.1CO} C .32300 .50 \mathrm{CCOC.C1h7}$

$8.00 \quad 0.10 C \quad 0.01300 .5 C C 0 \quad 0 .(167$

$10.00 \quad 0.100 \quad 0.01300 . E C L C$ C.C.1t.7

$12.000 .1000 .22320 .5000 \quad 0.0257$
$2.00 \quad 0.100 \quad 0.0150 \quad 0 . \varepsilon \in t 0 \quad 0.0167$

$4.00 \quad 0.1000 .01300 .46 \in C$ C.C167

$6.00 \quad 0.1 C C \quad 0.01300 .86600 .0168$

$8.00 \mathrm{C} .1 \mathrm{CC} 0.013 \mathrm{U} 0.8 \mathrm{E}$ u 0.0 .187

$10.00 \quad 0.100 \quad 0.31300 .66600 .0157$

$12.00 \mathrm{C.100} 0.131500 .806 \mathrm{COC} \quad 167$

2.00 C.1CC 0.0130 0.5ess C.0167

4.000 .1000 .01300 .46590 .0157

$6.00 \quad .1 C 0 \quad 0.3130 \quad 0.965,40.0167$

$8.00 \quad 0.100 \quad 0.3: 30 \quad 0.9659 \quad 0.0167$

$10.00 \quad 0.160 \quad 0.21300 .96590 .0157$

$12.00 \quad 0.100 \quad 0.02300 .5659 \quad 0.0167$
0.1422
0.2267
0.3005
0.3609
0.4343
0.4976

0.1148

0.1513

0.2389

0.2917

0.3420

0.3879

0.1026

0.1615

0.2120

0.2555

0.3020

0.3440

0.0981

0.1150

0.1014

$0 . \angle 332$

0.2766

0.3136

0.3462

0.1662

0.2063

0.3454

0.4124

0.4049

0.5218

0.2039

0.3258

0.4293

0.3135

0.5590

0.0545

0.2302

0.3728

0.4584

0.5564

0.0714

0.7484

0.0925

0.1450

0.1900

$0.23<1$

0.2748

0.3079

0.0919

0.1442

0.1860

0.2210

0.2378

U. 3244
0.2459

0.3991

0.5231

$0.0<90$

0.7224

0.8052

0.2508

0.4068

0.5334

0.0417

0.7374

0.0223
0.3645

0.6108

0.8403

1. $00 \cup 0$

1.0000

1.0000

0.3645

0.0196

0.84 ن 3

1.0000

i. 0000

1.0000

0.3645

0.6106

0.8403

$1.00 \mathrm{~N} 0$

1.0000

1.0000

0.3645

0.6108

0.8403

1. 0000

1.0000

1.0000
9.3670

12.14 .5

6. 357,

14.0143

15.3451

10.0135

10.4300

13.1223

11.0045

17.3743

22.5134

22.116 .5

10.91.1

13.7903

12.1033

19.3147

24.3210

24.1418

11.1576

14.1373

13.2420

$24.137 \mathrm{~s}$

25.30 .27

is. 2554

11.2304

14.3042

13.107:

20.5572

23.92 .1

$25.53 \times 2$

11.3101

14.34.07

13.315

$20.57=2$

23.9742

20.0027 


$$
D=0.15
$$


FLOH CIA. LSS. M. COEFF
SUPPLY EKAIN

SLCPE SLUPF (SIN) (SIN)
DRAIN FLCH

ENTRY DEPTH ENERGY

RATIO HIC.
H.
NORMAL DEPTH

HOD.
PIPE LENGTH T MORMAL DEPTH. $\angle 10$.

\begin{abstract}
$2.00 \quad .150 \quad c .0070 \quad 0.2568 \quad c .0500$
$4.00 \quad 0.15 C \quad 0.30+30.258$ E C.usnc

$6.000 .13 C \quad 0.20100 .2580 \quad . C 5 n 0$

$8.00 \quad 1.1500 .70700 .258 \mathrm{C} .0500$

$10.00 \mathrm{c} .1500 .3070$ n. $258 . \mathrm{C}$ C.c5nn

$12 . \operatorname{cu} 0.1500 .39700 .25660 .0500$
\end{abstract}

$2.00 \quad 0.150 \quad 0.0090 \quad 0.5000 \quad C .6500$

$4.000 .15 \mathrm{C} 0.90400 .500 \mathrm{C}$ C.6500

$0.00 \quad .150 \quad c .00 \rightarrow C \quad 0.5 C C C \quad C . C=00$

$8.00 \quad 0.15 C \quad 0.70+0 \quad 0.5 \operatorname{ccu} \quad c . c 500$

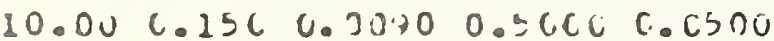

$12.00 \mathrm{C} .15 \mathrm{C} 0.00900 .50 \mathrm{Ccc} C .5 .500)$
0.0460
0.0710
0.0914
0.1104
0.1278
0.1439

0.0375

0.0577

0.0744

ט. .0944

0.1032

c. 1160

0.0337

$0.05 i 8$

0.0657

0. 0 Húa

0.0923

0.1038

0.0316

v. 0480

$0.00 \angle 0$

c. 0751

c. 0805

0.0772

0.0306

0.0470

0.0645

0.0726

0.0530

0.0738

0.0303

$0.04=5$

0.0594

0.0710

0.0527

0.0430
0.1973
0.3303
0.4433
0.5451
0.0355
0.7215

0.2919

0.4924

0.6659

0.0193

$0 .+605$

1. 0943

0.3592

0.0084

0.8244

1.0184

1.1944

1. 3600

0.4069

0.6099

0.9354

1. 1527

2. 3584

1.5492

0.4344

0.7373

0.198 d

1.1344

1.4545

1.0014

0.4426

0.7507

1. 1187

1.2593

$1.4 \mathrm{C} 44$

1.0917
0.0770

0.1201

0.1569

0.1901

0.2211

0.2507

0.0770

0.1201

0.1509

0.1901

0.2211

0.2507

0.0770

0.1201

0.1509

0.1 Yง 1

0.2211

0.2507

0.0770

0.1201

0.1569

0.1901

0.2211

$0.25 i 7$

0.0770

0. 1201

0.1509

0.1401

0.2211

$0.25 \mathrm{~J}$

0.0770

0. 1201

U. 2509

0.1901

0.2211

ง. 2507
17.4232

29.7004

41.1010

$50.371 / 5$

$5 y \cdot t 272$

60.5173

14.4303

33.3104

$46.6 J 27$

50.7011

t6.61

$70.40,2$

$20.26 j 7$

34.0491

47.8107

54.0542

69.2501

79.5004

20.6519

3y. 3224

$45.7=3$

6u. 1509

70.0539

81.0546

20.3545

35.6012

44.1602

ou. 7351

71.3110

61. 0443

$2 \mathrm{C} .9073$

$35.74>1$

44.2454

00. 서 7

71.5117

$0<.0347$ 


FLCH DIA. MAYN. SUFPLY CPA!P
LIS. M. COEFF SLCPE SLUPE
(SIN) (SIN)

CEAIN FLOH ENTRY ENTRY UEPTH EHERGY RATIT H/O.
NURMAL DEPTH $H / D$.
PIPE LEYUTH TI NORMAL UEPTH. L/U.

\begin{abstract}
$2.00 \quad 0.15 C \quad 0.0090 \quad 0.2580 \quad 0.0250$
$4.00 \quad 0.15 C \quad 0.7070 \quad 0.254 t \quad 0.0290$

t.00 C.15C u. 3n.30 $0.25 t \varepsilon \quad C .(2 \geq 50$

j.00 $\quad 1.1 \leq C \quad C .2070 \quad 0.25 \varepsilon E \quad C .(250)$

$10.00 \quad 0.150 \quad 0.01770 \quad 0.2 \leq \varepsilon 0 \quad(.0 ? 50$

$12.00 \quad 0.150 \quad 0.01) \rightarrow 0 \quad 0.256 t \quad c .0250$
\end{abstract}

0.0460
0.0710
0.0914
0.1154
$U .1275$
$v .1439$

0.0375

0.0577

0.0744

0.0844

0.1032

U.1100

0.0337

0.0516

U. $0 t_{-} 7$

0.0800

0.0723

4.1035

U. $032 \mathrm{C}$

$0.04 \div 6$

U. 0620

U. 0731

ט. 0305

0.0472

0.0360

0.0470

0.0605

$0.07 \angle 0$

U. $0 \times 3 t$

C.0.1E

0.0303

$0.0415=$

ن. $05: 4$

0.071 .

U. U⿺辶万 7

0.0430
0.8973

0.3303

0.4433

0.2451

0.0355

0.7215

0.2919

0.6659

0.0193

0.4000

1.3943

0.3592

$0.0 \cup 84$

$0 .: 244$

1.018 .4

1.1944

1.3600

0.4069

0.0 ช

0.4354

1.0527

1. 3584

1.2492

0.4344

0.7373

0.4589

1.2344

1.4545

1.0014

0.4426

c. 7,07

1. 0149

$1.2: 93$

$1.4: 44$

1.0417
0.0960

0.1508

0.1470

$0.24 j 6$

0.2510

0.3196

$0.09 \leftarrow 0$

0.1308

0.1976

0.2406

$0 . \overline{2510}$

0.3146

0.0900

$0.15 \cup 8$

0.1970

0.2400

$0.2+10$

0.3190

0.0900

0.1508

0.1976

0.2406

0.2010

J.3146

0.0900

0.1508

0.1476

U. 24.06

0.2310

J. 3146

0.0900

0.1508

0.1916

0.2446

ग. 2510

U. $31>0$
24.4377

$41.75,5$

$90 \cdot 3040$

64.0372

$61.7 \div 40$

$92.370 t$

20.1320

44.0034

QU. 2947

74.5432

c.7.01:3

44.5453

26.3259

40.0012

6..7942

70.0237

4i. $0,4=2$

I U 2 . j1 ij

$27.14: 2$

4u. 4 टuं

t<. 7412

71.0135

91.3214

10.7544

27.37 .7

40.7101

01.2J:3

7 $=$. C 114

प्रा. 1415

104.5100

$27.42,2$

4c. 317 )

0j. 32is

$7 c \cdot 3: 14)$

4... is s J

1C.4. 7 $5: 4$ 
FLOH DIA. MANN. L/S. H. CJEFF
SUPFLY TRAIN

SLCPE SLCPE (SIN) (SIN)
ORAIN FLUW

ENTRY DEPTH

PAT $10 \mathrm{H} / \mathrm{O}$.

\section{ENTRY \\ EMERGY \\ A.}

NORMAL

OEPTH

HOO.
PIPE LEVETA

NORMAL DEPTH. L/D.

\begin{tabular}{|c|c|c|c|c|}
\hline 2.00 & .150 & 0 . & & \\
\hline 4.00 & $0.15 \mathrm{C}$ & 0.2930 & $2 \xi t 6$ & 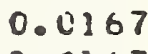 \\
\hline 6.00 & 0.150 & $6.20+0$ & 0.2580 & \\
\hline 8.00 & 0.150 & 0.30 .00 & $0.258 \bar{\varepsilon}$ & \\
\hline & $0.1>0$ & 170 & 0 & 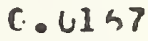 \\
\hline$\therefore 00$ & 0.130 & 10 & & \\
\hline 2.00 & 0. & 0. & & \\
\hline 4.00 & 0.1 & 070 & & \\
\hline 6.00 & 0.150 & 0.3030 & $\mathrm{CO}$ & c. \\
\hline & 0.150 & טדונים 0 & & \\
\hline 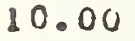 & C. 1 & $2>0$ & & \\
\hline$\bullet$ & C. 150 & 0.2370 & 0. & C. 0 \\
\hline
\end{tabular}

$2.00 \quad 0.150 \quad 0.0030 \quad 0.7 C 7 C \quad C . C 167$

$4.00 \quad 0.150 \quad 0.3090 \quad 0.7 c 7 C \quad 0.0167$

$6.00 \quad 0.150 \quad 0.2030 \quad 0.7670 \quad 0.0157$

$8.00 \quad 0.15 C \quad 0.03100 .7070 \quad 0.0167$

$10.00 \quad 0.1 \leq C \quad 0.0230 \quad 0.7 C .70 \quad c .0157$

$12.00 \quad 0.150 \quad 0.0070 \quad 0.7070 \quad 0.0167$

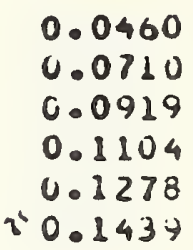

$2.00 \quad 0.150 \quad 0.20100 .9654 \quad 0.0167$

$4.00 \quad 0.150 \quad 0.00700 .46550 .0167$

$\begin{array}{llllll}6.00 & 0.150 & 0.2070 & 0.9 t 59 & 0.0167\end{array}$

$8.00 \quad 0.25 C 0.30700 .9454$ C.C167

$10.00 \quad 0.150 \quad 0.03100 .94590 .0 .167$

12.00

0.150

$0.9 t 59$ C.C.167
$0.9 t .54$ C. Cith?

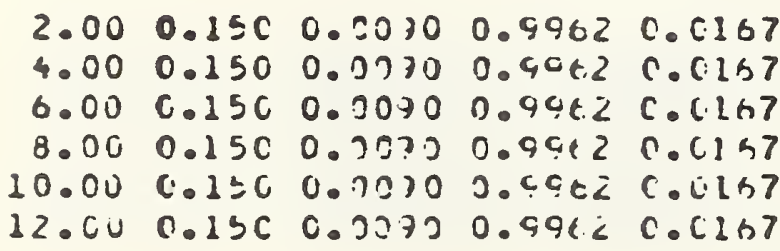

0.0375

0.0577

0.0744

0.0844

0.1032

0.1100

0.0337

0.0518

c. 0607

0.0500

0.0923

0.1038

0.0316

0.0436

0.0526

0.0751

0. 0305

0.0372

0.0306

0.1973

0.0303

0.0465

0.0549

0.0710

0.0327

0.0930
0.3303

0.4433

0.5451

0.0355

0.7215

0.2919

0.4924

0.6659

0.0193

0.9605

1.0943

0.3592

0.6084

0.6244

1.01184

1.1944

1.3606

0.4069

0.0889

0.9354

1.1527

1.3589

1.3482

0.4344

0.7373

0.4984

1.2344

1.4545

1.0614

0.1093

0.1725

0.2269

0.2766

0.3240

0.3694

0.1043

0.1725

0.2209

0.2706

0.3240

0.3544

0.1093

0.1725

0.2209

0.2700

0.3240

0.3694

0.1093

0.1725

0.2209

0.2760

ט. 3240

0.3644

0.1043

$0.17<5$

0.2264

0.2766

0.3240

0.3674

0.4426

0.7507

1.0184

1.2573

1.4244

1.0417
0.1043

0.1725

0.2209

0.2706

0.3240

$0.36 \% 4$
28.3700

48.0323

05.5370

79.5074

93.3925

106.0070

29.9749

$5 i .3733$

69.3127

84.2703

90.9729

$122.43: 1$

30.5473 52.5401

70.3004

80.2407

101.3507

115.1521

$31.008 i$

53.1465

71.7455

87. $32=5$

$102062 i 7$

120.5944

31.1056

53.4507

72 . $15: 6$

87.5043

103.2422

$117.315=$

31.2345

53.5303

$72.273 y$

$8=.3200$

103.42 .00

117.32 .14 
FlOH DIA. MANN. L/S. H. COEFF
SUPPLY ORAIN

SLCPE SLOPE

(SIN) ISIN]
ORAIN FLOH

ENTRY DEPTH

RATIO H/O.
ENTRY

ENERG

H.
MORHAL

DEPTH

M/O.
PIPE LEVGTH T

NCKMAL UEPTH. L/D.

\begin{abstract}
$2.00 \quad 0.150 \quad 0.0120 \quad 0.258 \varepsilon \quad 0.05 n 0$
$4.00 \quad 5.250 \quad 0.1120 \quad 0.2560 \quad 0.0500$

$6.000 .1500 .21200 .258 t \quad 0 . C 5 n 0$

$8.00 \quad 0.150 \quad 0.31200 .258 .60 .050 u$

$10.00 \quad 0.150 \quad 0.3120 \quad 0.258 t \quad 0.0500$

$12.0 \bar{u} \quad 0.1 \leqslant 0 \quad 0.01 \geq 0 \quad 0.2546 \quad 0.0500$
\end{abstract}

$2.00 \quad 0.150 \quad 0.01200 .50 .0 .6500$

$4.00 \quad 0.150 \quad 0.01200 .5600 \quad 0.0570$

$6.00 \quad 0.150 \quad 0.0120 \quad 0.50000 .0500$

$8.00 \quad 0.150 \quad 0.3120 \quad 0.5040 \quad 0.0500$

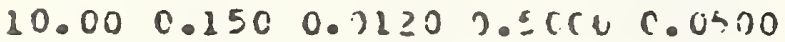

$12.00 \quad 0.150 \quad 0.31200 .506 U$ C.650

$2.00 \quad 0.15 C \quad 0.0120 \quad 0.70700 .0500$

$4.00 \quad 0.150 \quad 0.01200 .76700 .0500$

$6.00 \cdot 0.150 \quad 0.2120 \quad 0.70 .70 \quad 0.0500$

$8.00 \mathrm{c.15C} 0.22200 .7 C 740.0500$

$10.00 \quad 0.150 \quad 0.0120 \quad 0.7 C 70 \quad 0.0500$

$12.00 .0 .15 C \quad 0.0120 \quad 0.7070 \quad 0.0500$

$2.00 \quad 0.150 \quad 0.0220 \quad 0.8 t \in 0 \quad 0.0500$

$4.00 \quad 0.150 \quad 0.0120 \quad 0 . t e t U \quad 0.0500$

$6.00 \quad C .15 C \quad 0.01200 .8 t t . C \quad 0.0500$

$8.00 \quad 0.15 C \quad 0.01200 . \varepsilon t \in C \quad C . C 500$

$10.00 \quad 0.150 \quad 0.0120$ O.EC. ¿ 0.0500

$12.00 \quad 0.250 \quad 0.11200 .8660 \quad 0.0500$

$2.00 \quad 0.15 C \quad c .2120 \quad 0.9654 \quad 0.0500$

$4.00 \quad 0.150 \quad 0.3120 \quad 0.46590 .4500$

$6.00 \quad 0.150 \quad 0.11200 .9659 \quad 0.0500$

$8.00 \quad 0.150 \quad 0.3120 \quad 0.4 \in 590.4530$

$10.00 \quad 0.150 \quad 0.0120 \quad 0.9659 \quad 0.0500$

$12.00 \quad 0.150 \quad 0.0120 \quad 0.9659 \quad 0.0509$

$2.00 \quad 0.150 \quad 0.0120 \quad 0.99620 .0500$

$4.00 \quad 0.150 \quad 0.31200 .99620 .45018$

$6.00 \quad 0.150 \quad 0.0120 \quad 0.9462 \quad 0.0500$

$8.00 \quad 0.15 C \quad 0.0120 \quad 0.9962 \quad 0.0502$

$10.00 \quad 0.15 C$ C. C. $1200.940=$ C. 6500

$12.00 \quad 0.150 \quad 0.0120 \quad 0.4942 \quad 0.0500$
0.0550

0.0951

0.1204

0.1332

0.1544

0.1742

0.0440

0.0692

0.0894

0.1075

0.1244

C. 1400

0.0403

0.0620

0.0300

ن. 0403

U. 1110

0.1251

0.0378

0.0501

0.0751

0.0931

0.1041

0.2170

0.0305

0.0562

c. 0720

$0.087 i$

0.1004

0.1130

0.0362

0.0537

0.0710

4.0002

$0.09+4$

c. 1119
0.1413

0.2350

0.1139

0.3033

0.4454

0.5039

0.2071

0.3472

0.4667

0.2739

0.0093

0.7605

0.2546

0.4290

0.378 .1

0.1097

0.0334

0.5440

0.2873

0.4253

0.6533

0.0064

0.4453

1. 4765

0.3073

0.5165

0.3991

0.0025

1.0138

1.1524

0.3123

0.3277

0.7131

0.0792

2.0333

1.1751
0.0925

0.2449

0.1901

0.2311

0.2648

0.3064

0.0425

0.1449

0.1901

0.2311

0.2648

0.3004

0.0925

0.1449

0.1901

0.2311

$0.269 y$

0.3004

0.0925

0.1449

0.1901

0.2311

0.2598

0.3004

0.0925

0.1449

0.1901

0.2311

0.2648

0.3064

0.0925

0.1449

0.1901

0.2311

$0.20 y 5$

0.3064
11.5856

20.110 .0

$27.040 j$

35.9407

$4 u \cdot 11062$

43.1237

13.2442

22.4141

36.7705

31.3721

44.6241

5i. 3005

13.7751

23.3320

32.0150

$39.3 \times 42$

40.4321

52.3035

14.0445

25.7953

32.6333

$40.1 \pm 49$

$47.30>2$

53.4451

14.1313

24.3203

$3<.44,1$

40.5714

41.5343

53.9125

14.2135

24.0334

$3 j .03,3$

4 4.. 7.2

47.7 i j

54.1133 


\begin{tabular}{|c|c|c|c|}
\hline $\begin{array}{l}F L \cap H \\
\text { L/S. }\end{array}$ & 014. & $\begin{array}{l}\text { MAYY. } \\
\text { CDEFF }\end{array}$ & $\begin{array}{l}\text { SUFPLY } \\
\text { SLCPE } \\
\text { (SIN) }\end{array}$ \\
\hline
\end{tabular}

CKAIV FLCH

ENTRY UEPTH RAII:T H/C.
ENTRY

ENERGY

M.
NURAAL DEPTH H/O.
PIPE LENETH TI MORMAL JEPTH. LID.

\begin{abstract}
$2.00 \quad 0.15 C \quad 0.0120 \quad 0.2582 \quad 0.0250$
$4.00 \quad 0.150 \quad 0.11200 .256 t \quad 0.0250$

$6.00 \quad c .1=0 \quad 0.11200 .2 \leq 20 \quad c . c 250$

$8.00 \quad 0.150 \quad 0.21200 .25 L C$ C. 2559

$10.000 .15 C \quad 0.21 \geq 0 \quad 0.2 \pm-0 \quad \because .02 \geq 50$

12.000 .150 c.J120 .025 t C C.0250
\end{abstract}

\begin{abstract}
i. 0550
0.0321

U. 1104

0.1352

0.1544

0.2742

0.0448

0.0642

$v .0344$

U. 1073

v. 1244

0.1400
\end{abstract}

0.0403

$0.05<0$

0.0300

0.0903

C. 1110

0.1251

0.0370

$0.05,1$

0.0751

U. 0701

0.1041

0.1170

(.. 030 s

0.0532

c. 0720

U. OE

0. 10 (i)

0.1130
0.1413

0.2350

0.1139

0.3533

0.4454

0.5039

0.2071

0.3472

0.4667

c. $>7 ? 9$

0.0693

$0.7 \in 05$

0.2346

0.4290

0.2731

0.7004

0.0334

0.9448

$0 .<x>3$

0.4053

$0.0>33$

0.0064

0.4453

1. 0705

0.3073

0.5165

0.0791

$0.50<3$

1.0135

1.1528

U.03t:

0.0527

$0.07 i=$

C.Cto:

w.

U.11:4

.0 .3123

0.3277

0.7131

$0 . .7742$

1. 1333

1. 1751
0.1255

0.1825

$0.24 \cup 6$

0.2442

U. 3445

0.3913

0.1135

0.1325

0.2406

0.2742

0.3445

J. 3933

0.1135

$0.13<5$

$0.24 \cup 6$

0.2442

0.3445

0.3933

ن. 1155

0.11325

$0.24 \cup 6$

0.2942

0.3443

0.3433

0.1125

0.1025

0.2406

0.2742

J. 344 J

J.3453
0.1155

0.1525

$0.24 \cup 6$

0.21342

0.3445

v. 34,3
$15.70<3$

26.7455

$30.17<0$

$44.5 .8 \% 4$

51.454 .03

$50.50 v 4$

10.8157

$2 c .0435$

$30.01 ; 5$

47. ह7ว

53.3104

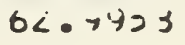

17. $43<9$

29.jうर

34.9007

4บ. 2074

57.0003

64. 4432

17.6544

24.9001

$40.54<0$

SU: 140

57.7502

0.

17.7543

$30.1=50$

40.4431

उᄂ. 3020

20. 4013

00.42 .4

17. .120

30.2415

4u.92

=-4.

E: :

ou. $5=: 2$ 
FLOM DIA. MANN. SUPPLY TRAIN L/S. M. CIJEF SLOPE SLGPE (SIN) (SIN)
DRAIN FLOH ENTRY UEPTH RLTIO HID. .
ENTRY

ENERGY

ก.
NORMAL

DEPTH

MO.
PIPE LEICTH I

NORMAL DEPT.H. L/O.

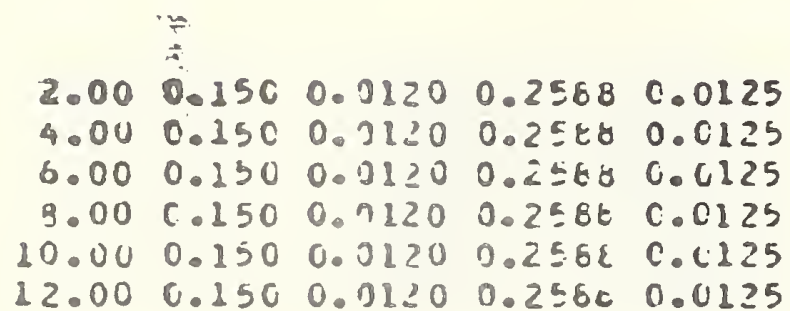

$\begin{array}{rllll}2.00 & 0.150 & 0.0120 & 0.50 C C & 0.0125 \\ 4.00 & 0.150 & 0.3120 & 0.5000 & 0.0125 \\ 6.00 & 0.150 & 0.3120 & 0.5006 & 0.0125 \\ 3.00 & 0.150 & 0.3120 & 0.50 C U & 0.0125 \\ 10.00 & 0.150 & 0.2120 & 0.5 C C C & 0.0125 \\ 12.00 & 0.150 & 0.11120 & 0.5000 & 0.0125\end{array}$

$2.00 \quad 0.150 \quad 0.01200 .7070 \quad 0.0125$

$4.00 \quad 0.150 \quad 0.01200 .7070 \quad 0.0125$

$6.00 \quad 0.130 \quad 0.01220 .7070 \quad 0.0125$

$8.00 \quad 0.150 \quad 0.31200 .7070 \quad 0.6125$

$10.00 \quad 0.15 C \quad 0.01200 .70 .20 \quad 0.0125$

$12.00 \quad 0.150 \quad 0.71200 .7070 \quad$ C. 0125
0.0550
0.0551
0.1104
0.1332
0.1544
0.1742

0.0448

0.0642

0.0894

0.1075

0.1244

0.1400

\subsection{3}

0.2350

0.3139

0.3833

0.4454

0.5039

0.2071

0.3472

0.4667

0.2739

0.4093

0.7005

0.0403

0.0620

$0.08 \cup 0$

0.0953

0.1110

0.1251

0.0378

0.0531

0.0751

0.0701

0.1041

0.1170

0.0365

U. 0562

ن. 0726

0.0572

0.1004

0.1130
0.2546

0.4290

0.5781

0.7099

0.0334

0.4448

0.2873

0.4853

0.0533

0.0064

0.1453

1.0765

0.3073

0.5186

0.0991

0.5625

1. 3338

$1.1>25$
0.1449

0.2311

0.3064

U. 3707

0.4436

0.3003

0.1449

0.2311

0.3004

0.3707

0.4436

0.5083

0.1449

0.2311

0.3004

0.3707

0.4430

0.5003

0.1449

0.2311

0.3004

0.3707

0.4436

J. $50: 3$

0.1449

0.2311

0.3004

0.3707

j. 4436

0.5003

0.0362

0.0557

0.0718

0.0502

0.0994

0.1119
0.3123

0.3277

0.7131

0.0792

1.0333

1. 1751
0.1449

0.2311

0.3004

0.3707

0.4436

U. 3003
10.0761

30.4910

40.2251

4 t. 6052

53.6057

61.0545

14.1021

32 .354

42.7044

52.5045

59.39 .7

66.01321

14.6425

33.1877

$43.92 \pm 0$

53.2042

60.3500

67.5715

19.8956

39.1313

45.0325

$31.04+1)$

60.1597

$67.7=17$

20.0315

$204.50: 3$

45.1472

$44.7: 103$

59.7624

67.0375

20.1121

120.3570

40.2104

44.3237

54 .6丁 3

67.55io 


FLOH CIA. MANN. SUPPLY TRAIN
L/S. M. CUEFF SLCPE SLUPE
(SIN) ISINI

JRAIN FLOH

ENTRY UEPTH

RATIO H/C.
EATRY

ENERGY

M.
MOKMAL

DEPTH

H/D.
PIPE LE OGTA TL NOKMAL JEPTH. L/D. $\begin{array}{lllll}2.00 & 0.150 & 0.0120 & 0.2560 & 0.0167 \\ 4.00 & 0.150 & 0.0120 & 0.2560 & 0.0147 \\ 6.00 & 0.150 & 0.1120 & 0.2566 & 0.1157 \\ 8.00 & 0.156 & 0.1120 & 0.2582 & 0.0167 \\ 10.00 & 0.150 & 0.0120 & 0.2560 & 0.0167 \\ 12.00 & 0.150 & 0.0120 & 0.2546 & 0.0167\end{array}$

$2.000 .1500 .01210 .5 c 400.0167$.

$4.00 \quad 0.150 \quad$ C. $.120 \quad 0.500 C$ C. 0.157

$0.00 \quad 0.15 C \quad 0.121200 .5 C C O C .0167$

$6.50 \quad 0.150 \quad 0.31200 .5 C(C)$ C.C167

$10.00 \quad 0.150 \quad 6.3120 \quad 0.5$ C.C.C. C.C157

$12.00 \quad c .150 \quad 0.2120 \quad 0.3$ CC.C 0.0167

$2.00 \quad 0.150 \quad 0.0120 \quad 0.707 C \quad$ C.CLA

$4.00 \quad 0.1 \leq 0 \quad 0.01200 .7 C 7 C \quad 0.0147$

$6.00 \quad 0.150 \quad 0.91200 .7 C 70 \quad 0.0157$

$8.05 \quad 0.250 \quad 0.21200 .7074 \quad$ C.C16.7

$10.00 \quad 0.1 \leqslant 0 \quad 0.2120 \quad 0.76700 .0137$

$12.00 \quad 0.150 \quad 0.01200 .707 \mathrm{C}$ C.CL67

$2.000 .150 \quad 0.01200$. EteC 0.0167

$4.00 \quad 0.15 C \quad 0.21200 . E \in C O C . C 167$

$0.00 \mathrm{C} .15 \mathrm{C} \quad 0.0120 \quad 0.8 C t C \quad \mathrm{C} . \mathrm{C} 167$

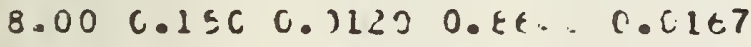

$10.00 \mathrm{C} .15 \cup 0.11 \geq 0$ D. $26 \in 0$ C. $C 16.7$

$12.00 \quad 0.150 \quad 0.31200$. ettu C.0167
4.0550
U. 0551
c. 2104
0.1332
ن. 1544
0.2742

$0.0440^{\circ}$

0.0692

v. 0344

0. 1075

0.1244

$0.1400^{\circ}$

0.0403

i. 0620

U. $0 \equiv 00$

c. 0903

0.1110

0.1251

$0.037=$

ง. 0531

0.0751

0.0901

0.1041

U. 1170

0.0305

0.0502

0.0720

U.0.5/ 1

U. 1004

ᄂ.113u

2. 0352

c. $0=5$ ?

0.0710

U. 0:- 42

0.01 .14

i. 1124
0.1413

0.2350

0.3137

0.3533

0.4454

0.5037

0.2071

0.3472

0.4667

0.5730

0.0693

0.7005

0.2540

0.4290

0.2781

0.7099

0.8334

0.7448

$0 .<873$

$0.4 C 53$

0.0533

$0 . \simeq 064$

0.4453

2. 4705

0.3073

0.ร18

0.0971

0.0625

1. 135

1.1525

0.3121

$0.22: 7$

0.7331

$0.0: 42$

1.0333

1.1751

\subsection{0 \\ 0.2044 \\ ن. 2760 \\ $0.33 \% 1$ \\ 0.3917 \\ 0.4558}

$0.13<0$

0.2094

0.2706

0.3391

0.3907

0.4258

0.1320

$0.20+4$

0.2706

$0.33: 1$

0.3957

0.4520

0.1320

0.2044

0.2700

0.3341

ט. 3†० 7

0.4508

0.1320

0.2094

0.2766

U. 33.1

$0.39,7$

ن. 4500

$0.13<0$

$0.213+4$

0.2706

0.33 .2

0.3907

0.4250
17.7316

24.7515

34.3035

$47.35,73$

500351

62.054 )

10.3126

31.6205

$4 i .9321$

5 i. 346.7

$5 y .3314$

60.3107

$14.2=35$

32.4445

$43.200 \%$

ะட.4ंบ

01.0103

$6 c .29,3$

14.5303

32.074 \%

43.5200

$51.24 \geq 2$

OL.tis 1

64. $33=2$

14. serso

उ3. 1) 1

4 $30.12+1$

ن

c 2.3740

7 …17

L., $t+1,3$

32.247 ,

44.11414

$23.7 \ldots 3$

can $1<13$

$7 \mathrm{i}=\mathrm{No}$ 
FLON OSA. MANA. SUPPLY OKAIM
LIS. M. GJEFF SLEPE SLOPE
(SIN) (SIM)
DRAIN FLOH ENTRY DEPTH RAT 10 H/O. .

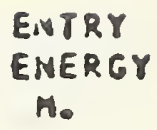

MORML DEPTH H/O.

$\therefore$

.2

\begin{abstract}
$2.00 \quad 0.150 \quad 0.2150 \quad 0.2568 \quad 0.4500$
$4.00 \quad 0.150 \quad 0.0150 \quad 0.2586 \quad 0 . i 5 n 0$

$6.00 \quad 0.15 C \quad 0.0150 \quad 0.25 t a \quad c .05 n 0$

$8.00 \quad 0.150 \quad 0.2150 \quad 0.258 E \quad C . C 500$

$10.000 .150 \quad 0.01500 .2566$ C. 1500

$12.00 \quad 0.150 \quad 0.0150 \quad 0.2586 \quad 0.0500$
\end{abstract}

\begin{abstract}
0.0633
0.0962

0.1278

0.1544

0.1791

0.2025

0.0515

0.0797

U. 1032

0.1244

0.1434

0.1022
\end{abstract}

\section{$2.00 \quad 0.15 C \quad 0.0150 \quad 0.7 C 7 C \quad 0.0500$ \\ $4.00 \quad 0.15 C \quad 0.31500 .7070 \quad C .6500$ \\ $6.00 \quad 0.130 \quad 0.2150 \quad 0.7070 \quad C .0(1 \div 00$ \\ $8.00 \quad 0.15 \mathrm{C} \quad 0.0250 \quad 0.7070 \quad 0.5500$ \\ $10.00 \quad 0.250 \quad 0.2250 \quad 0.7070 \quad$ C. $v 51,0$ \\ $12.00 \quad 0.130 \quad 0.151500 .7070 \quad 0.65 .20$}

\subsection{2 \\ 0.0714 \\ $0.04<3$ \\ 0.1110 \\ 0.12 क3 \\ 0.1447}

0.0434

0.007 i

0.0305

ن. 1341

0.1202

0.1354

c. 0420

v. 0546

ن. $03 j 6$

0.1024

6.1100

0.13157

0.0415

0.0640

0.0527

i. 0494

c.1149

$0.12 ; 3$
0.1100

0.1618

0.2411

0.2934

0.3400

0.3839

0.1597

0.2659

0.3557

0.4352

0.0076

0.3752

0.1953

0.3271

0.4389

0.5394

0.0309

0.7146
0.1068

0.1681

0.2211

0.2598

0.3157

ט. 3596

0.2008

0.1601

0.2211

0.2648

0.3127

0.3596

0.1068

0.1601

0.2211

$0.26 \div 8$

0.3157

0.3546

0.2201

0.3094

0.4975

0.0100

0.7151

0.0114

0.2350

0.3953

0.5317

0.0542

0.7552

0.0670

0.2397

0.4026

0.0423

0.0667

$0.7 E 21$

0.0869
0.1068

0.1531

0.2211

0.2698

0.3157

0.3596

0.1008

0.1601

0.2211

j. 2645

$0.31>7$

0.3546

0.1008

0.1001

0.2212

0.2698

$0.31>7$

0.3546
PIPE LENCTA T NORAAL DEPTH. LIO.

\author{
8.6502 \\ $14.626 j$ \\ 14.7675 \\ 24.4114 \\ 20.5509 \\ $3<.2704$
}

9.0042
16.2471
21.9704
27.1350
31.7370
35.1110

9.9902

16.9140

22.8007

20.2620

$33.07 j 2$

37.4100

1C. 1453

17.2543

23.3523

20.3370

33.7425

$30.1+36$

10.2574

17. 43.52

23.5053

29.1343

$34.0 \leq 03$

$30.27=3$

10.3200

17. 4.700

$23.55 \angle 5$

$24.2 i<J$

34.1 Cs 3

$30.03,3$ 
FLOH OIA. MAVN. SUPFLY C.FLIT L/S. P. GOEFF SLOPE SLLPE (SII)' (SIN)
DRAIN FLCH ENTRY DEPTH RATIU M/D.
ENTRY ENERGY

M.
NURMAL DEPTH

$M / D$.
PIPE LEMITH NORAAL UEPTH L/D.
$2.00 \quad 0.150 \quad 0.0150 \quad 0.258 E \quad 0.0250$ $4.00 \quad 0.150 \quad 0.01 j 30.2556 \quad 0.6250$ $0.00 \quad 0.150 \quad 0.1250 \quad 0.258$ C C. (250) $8.00 \quad 0.1 \leq 0 \quad 0.11200 .258$ r. C. 10.0u r. $15 C \quad 0.0150 \quad 0.2 E \varepsilon_{0} \quad$ r. C C 250 $12.000 .150 \quad 0.31200 . \overline{2}$ SEt 0.0250

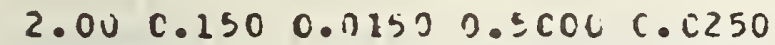
$4.00 \quad 0.150 \quad C .11500 .5 C(C \quad C .0250$ $6.00 \quad 0.15 C \quad 0.0150 \quad 0.5 C C O C .0250$ $8.00 \quad(.1500) 1500.5 C C C \quad. C . C 2 \leq 0$

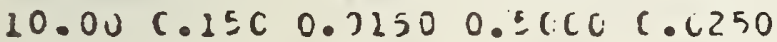
$12.00 \mathrm{C} .1 \leq \mathrm{C} 0.31500 .5 \mathrm{CCC} \quad \mathrm{l} .0250$

$2.00 \quad 0.150 \quad 0.0150 \quad 0.7670 \quad 0.0750$ $4.00 \quad 0.1 E C \quad G .11300 .7076$ C.0.250 $6.00 \quad c .15 C \quad 0.0150 \quad 0.707 C \quad C .6250$ $8.00 \quad 0.15 \mathrm{C} \quad 0.31530 .7 \mathrm{C}$ C C.4250 $10.00 \quad 0.15 C \quad 0.31510 .7 C 70 \quad$ C.C. 0.50 $12.00 \quad 0.250 \quad 0.3150 \quad 0.7070 \quad c . C 250$

$2.00 \quad 0.150 \quad 0.0150 \quad 0 . E f, E C \quad 0.0250$ $4.00 \quad 0.1 \leq C \quad C .31500 .2 E C C \quad 0.0250$ $6.00 \quad 0.15 C \quad 0.3150 \quad 0.8 t \in 0 \quad 0.0250$ 8.c0 C.iso (1.)150 O.tetC C.C(251) $10.00 \quad 0.1=C \quad 0.0150 \quad 0 . E \in C C \quad 0.0250$ $12.00 \quad 0.15 C \quad 0.3150 \quad 0$. tetC C.C.250

0.0633
0.0902
0.1275
0.1544
0.1791
0.2025

0.0515

0.0797

C. 1032

0.1244

0.1439

0.1522

0.0462

c. 0714

0.0 .123

$0.111 i$

0.1203

0.1447

0.0434

0.0070

0.0505

0.1041

0.1232

0.1354

$0.04 \leq 0$

0.0640

U. 0.336

0.1004

ن. 1100

U. 1307

0.0425

C. $054 \mathrm{U}$

0.0327

0.0 .714

11. 1140

0.1243
0.1100

0.1819

$0 .<411$

$0 .<734$

0.3403

0.5837

0.1597

0.2659

0.3557

0.4351

0.5070

0.5752

0.1953

0.3271

0.4389

0.2394

0.030 .1

0.7146

0.2201

0.3094

0.4975

0.0100

0.7151

0.0114

0.2350

0.3953

0.5317

0.0542

0.7652

0.0678

0.2397
0.4526
0.5423
0.460 .7
0.7011
0.0869

0.2397

0.1337

0.2263

0.2610

ن. 3445

j. $4 u=0$

v.
10.7377

10.0057

24.1442

29. 2010

33.7547

37.5657

11.5501

19.4014

$20.0=19$

$31.5 \div 212$

30.5530

$40.779 \mathrm{~J}$

11.9141

26.5705

$20.907 \rightarrow$

32.2475

37.0246

$42.21 \mathrm{JO}$

12.1030

$20.4 \mathrm{i} 61$

27.3014

33.20 .14

30.4704

$4<.50>0$

$1<.23,0$

$2 i .5 \sec 3$

$27.5: 72$

$33.4+21$

$3 c .=0.4$

43.3400

1C.2230

टи०गز:0

27.0720

$3 \equiv .21013$

30. त

$4: .4004$ 


\begin{tabular}{|c|c|c|c|c|c|c|c|c|c|}
\hline $\begin{array}{l}\text { FLOH } \\
\text { L/S. }\end{array}$ & DIA. & $\begin{array}{l}\text { MANV. } \\
\text { COEFF }\end{array}$ & $\begin{array}{l}\text { SUPPLY } \\
\text { SLOPE } \\
\text { CSINI }\end{array}$ & $\begin{array}{l}\text { RRAIN } \\
\text { SLCPE. } \\
\text { (SIN) }\end{array}$ & $\begin{array}{l}\text { DRAIN } \\
\text { ENTRY } \\
\text { RATIO }\end{array}$ & $\begin{array}{l}\text { FLOH } \\
\text { DEPTH } \\
\text { H/D. }\end{array}$ & $\begin{array}{l}\text { ENTRY } \\
\text { EHERGY } \\
\text { H. }\end{array}$ & $\begin{array}{l}\text { NORMAL } \\
\text { OEPTH } \\
\text { H/D. }\end{array}$ & $\begin{array}{l}\text { PIPL LEYGTH } \\
\text { NORMAL UEPT } \\
\text { L/D. }\end{array}$ \\
\hline
\end{tabular}

$\begin{array}{rlllll}2.00 & 0.150 & 0.0150 & 0.2586 & 0.0167 & 0.0633 \\ 4.00 & 0.150 & 0.1150 & 0.2586 & 0.0167 & 0.0902 \\ 6.00 & 0.150 & 0.0150 & 0.2588 & 0.0167 & 0.1276 \\ 8.00 & 0.150 & 0.0120 & 0.2588 & 0.0167 & 0.1544 \\ 10.00 & 0.150 & 0.1150 & 0.2580 & 0.0167 & 0.1771 \\ 12.00 & 0.15 C & 0.1150 & 0.2560 & 0.1167 & 0.2025\end{array}$

0.1100

0.1818

0.2411

0.2934

0.3400

0.3839

C. 0515

0.0747

0.1032

0.1244

0.1439

0.1522

$10.00 \quad 0.150 \quad 0.3150 \quad 0.500 C \quad 0.0137$

$12.00 \quad 0.150 \quad 0.3120 \quad 0.5000 \quad 0.0157$

0.1597

0.2659

0.3557

0.4351

0.5075

0.5752
0.0462

0.0714

0.0923

0.1110

0.1203

0.1447

0.0434

0.0670

0.0865

0.1041

0.1202

0.1354
0.4384

$0.04=0$

0.0640

c. 08336

0.1004

0.1160

0.1307

$10.000 .15 C \quad 0.01500 .9659 \quad$ C.013)

$12.00 \quad 0.15 C \quad 0.0150 \quad 0.0459 \quad 0 . C 167$
0.0425

0.0040

0.0327

0.0944

0.1143

0.1273
0.1953

0.3271

0.5394

0.0309

0.7146

0.2201

0.3694

0.4475

0.0100

0.7151

0.8124

0.1527
0.2438
0.3240
0.3967
0.4700
0.5386

11.0444

10.3513

24.0826

26.7155

32.5012

35.4802

0.1527

0.2438

0.3240

0.3957

0.4700

0.5306

21.3447

$19.727 \mathrm{~J}$

25.974t

31.0713

35.2315

30.5815

$0.15 \angle 7$

0.2438

0.3240

0.3957

$0.47 \cup 0$

0.5356

12.2030

20.3510

20.0202

32.1454

36.5600

40.1303

0.1527

0.2438

0.3240

0.3907

0.4700

0.5306

12.3439

20.6810

$27.20 \% 4$

32.7123

37.2305

40.4677

0.1527

.0 .2438

0.3240

0.3407

$0.47 \cup 0$

$0.53=6$

12. 4บบบ9

$20.5>47$

$2 \pi .32 .4$

33.2450

31.3540

41.3034

0.0678

0.2397

0.1527

0.2436

12.5210

0.4026

0.5423

0.0667

0.3240

0.39 .7

0.4700

0.5300
20.9003

27.5877

33.3315

20.9021

41. 5145 


FLOH CIA. MANN.
LIS. M. MOSFF

SUPPLI ORAIN

SLCPE SLLPS

(SIN) (SIN)
DRAIN FLCH

EATRY DEPTH

RATIO H/O.

\author{
ENTEY \\ ENERGY \\ H.
}

NORMAL DEPTH

H/O.
PIPE LEVGTH T NORMAL DEPTH. L/D.

\begin{abstract}
$2.00 \quad 0.150 \quad 0.0150 \quad 0.2586 \quad 0.0125$
$4.00 \quad 0.150 \quad 0.3250 \quad 0.5588 \cdot c . c 1 \geq 5$

$6.00 \quad 0.15 C \quad 0.0130 \quad 0.2560 \quad C . C 17.5$

$3.00 \quad 0.150 \quad 0.21500 .25 \varepsilon \theta \quad 0.0125$

$10.00 \mathrm{C} .150 \quad 0.01 .000 .2580 \mathrm{C.C}(1.5$

$12.00 \quad 0.15 C \quad 0.01500 .2580 \quad 0.0125$
\end{abstract}

$2.00 \quad 0.150 \quad 0.3150 \quad 0.5000 \quad 0.0125$

$4.00 \quad 0.15 C \quad C .3150 \quad 0.5 C C O \quad 0.4125$

$6.00 \quad 0.150 \quad 0.2150 \quad 0.5 C G U C .0125$

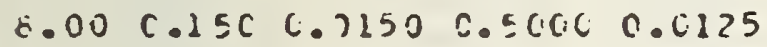

$10.00 \quad C .15 C \quad 0.0250 \quad 0.5 C C C \quad 0.0125$

$12.00 \quad 0.150 \quad 0.3152 \quad 2.50 C 0 \quad 0.0125$

\begin{abstract}
0.0633
0.0762

$0.1270^{\circ}$

0.1544

0.1791

0.2025
\end{abstract}

0.0515

0.0797

0.1032

0.1244

0.1439

0.1022

0.0462

0.0714

0.0723

0.1126

0.1233

0.1447

0.0434

0.0670

0.0205

U. 1042

U. 1202

v.1324

0.0420

0.0040

v. 0.333

U. 1304

ci. 1160

ט. 1347

0.0425

0.0 .640

c. 0327

J. . .1.14

U.114=

$0.12+3$

\subsection{0}

0.16 .18

0.2411

$0 . \angle 934$

$0 . j 4 \bar{s}$.

0.3839

0.1597

0.2657

0.3557

0.4351

$0.5 \cup 70$

0.5752

0.1953

0.3271

0.4389

0.3394

0.030 t

0.7146

0.2201

0.3694

$0.497 j$

0.0105

0.7151

C. $=114$

0.2350

0.3453

0.5317

0.0542

$0.76=2$

$0.007=$

0.2397

0.4020

0.3423

$0 . \operatorname{cts} 7$

$0.7=11$

0.0864
0.1601

0.2699

U. 3596

0.4436

0.5239

0.6021

0.1601

0.2698

0.3596

0.4436

0.5239

0.6021

0.1601

0.2698

0.3540

0.4430

0.5239

0.6021

$0.16=1$

$0.26 \div 8$

0.3596

0.4436

U. 5239

0.6021

0.1601

0.2693

0.3546

v. 4430

0.5239

$0.00<1$

0.1001

0.2698

0.3596

0.4436

ग. 2.239

0.0021
10.3002

16.9455

21.65 .14

24.9771

$27.5=14$

36.1773

11.1153

16. 3109

23.6000

27.3475

30.3022

$3<.6502$

12.4320

10.7752

24.4751

$26.44<0$

31.ดก5ร

33.4417

11.0702

14.2507

24.8705

29. 1034

$3<03=30$

34.0345

$11.93+1$

19. 3 5०

23. ग0

$24 \cdot 32-3$

$3<070=3$

$34.4+51$

16.9.100

[Y. 315.3

23. 1240

24.40133

3द. . LoJ

$9=.1037$ 


\section{A. MANY. SUPPLY TEAIN M. COEFF SLCPE SLCPE (SIN) (SIN)}

ORAIN FLOM ENTPY JEPTH RAIIO H/D.
ENTRY ENERGY

ค.
NORMAL DEPTH

H/O.
PIPE LENGTH TC NIJRMAL UEPTH. L/O.

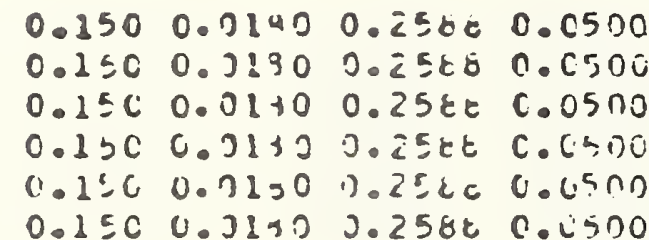

$0.15 C 0.01900 .5 C C C$ C.0500

$0.150 \quad 0.91300 .5000 \quad c .0500$

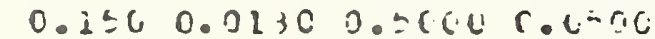

$0.1500 .31,00.5 C(C \quad$ c.ijon

0.1560 .21300 .50 .00 C. 0.500

00.1500 .01300 .5060 C. 6500
0.0710
0.1144
0.1439
0.1742
$0.20<5$
0.2294

0.0577

$0.00+4$

(.. 1100

0.1400

G. 1022

U. 1035

0.0518

0.3200

0.1036

0.1251

0.1447

0.1032

0.0486

$0.07 \leqslant 1$

U. 0472

0.1170

0.1354

0.1527

c. 0470

0.0720

0.0338

4.1130

0.1307

0.1473

0.0405

0.0710

4.0930

0.1114

4.1293

0.1454
0.0906

2.1487

0.1966

0.2393

0.2759

0.3100

0.1296

0.2149

0.2666

0.3497

0.4069

0.4582

0.1579

0.2636

0.3513

0.4303

0.2025

0.5685

0.1777

$0 . \angle 906$

0.3960

$0.4=2$

0.5097

0.0445

0.1296

0.3163

0.4257

0.5215

0.0055

0.0894

0.1929

0.3229

0.4334

0.2315

0.0228

$0.7 \cup 32$
0.1201

0.1901

$0.25 \cup 7$

0.3004

0.3546

0.4104.

0.1201

0.1941

0.2507

0.3004

0.3540

0.4104

0.1201

0.1901

0.2507

0.3004

0.3596

$0.41 \cup 4$

0.1201

0.1901

0.2507

0.3004

0.32 .16

$0.41 \cup 4$

0.1201

0.1901

0.2507

$0.30=4$

0.3540

$0.41 \cup 4$

0.1201

0.1901

0.2507

0.3004

0.3596

0.4104
0.4405

11.0007

14.7576

$1 . .0002$

20.9125

23.4247

7. 1413

$12.19=5$

10.3512

14.2475

$23.25 i 2$

<0. $30=7$

7. 4375

$12.7 D=4$

17.0051

20.7004

24.2570

$27.22=0$

7.5925

12.7649

17.4210

21. 1571

24.7300

27.0241

7.6719

13.0745

$17.01=7$

2i. 3.353

23.0474

$25.13=0$

7.3924

10.137

17.30s2

$21.44,7$

$2=013,2$

$20.21+2$ 


FLOH CIA. MAYN. SUPPLY CFAIN
LS. H. COEFF SLCPE SLLPF
(SIN) (SIIT)

$2.00 \quad 0.150 \quad 0.13130 \quad 0.2560 \quad 0.0250$ $4.00 \quad 0.130 \quad 0.31350 . z 5 E$ C. . 250 6.OC $0.150 \quad$ (.) $130 \quad 0.25 t 6 \quad(.1)>$. $8.00 \quad(.150 \quad 0.313 .30 .25=0 \quad C .0250$ $10.06 \quad 0.150 \quad 0.11300 .85680 .0250$ $12.00 \quad 0.1>0 \quad 0.3210 \quad 0.2560 \quad 0.0350$

$2.00 \quad 0.25 C \quad 0.01300 .5 C c C \quad 0.0250$ $4.00 \quad 0.150 \quad c .3230 \quad 0.3000 \quad(.0 .250$ $6.00 \quad 1.1 \leq 0 \quad 0.31300 .5 C C 0 \quad$ C.C?55 $8.00 \quad c .150$ C. $31100.5 C(L$ C.C $25 n$

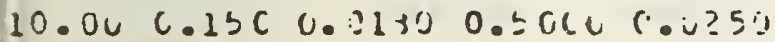
$12.0 \mathrm{C}(.150 \mathrm{C.}) 1 \times 2.0 .5 \mathrm{ClC}(.0 .250$

$2.00 \mathrm{C.15C} 6.31300 .7 \mathrm{C}$ (4)

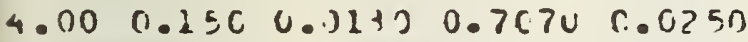

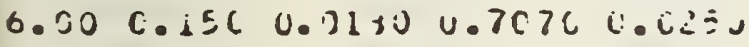
$\therefore .00 \quad 0.1 \pm C \quad 0.3130 \quad 0.7074 \quad . .4250$ $10.00 \quad 0.1 \leq 0 \quad 0.0110$ n.7CTC C..visc

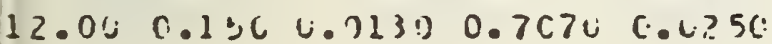

$2.00 \quad 0.130 \quad 0.01300 . t t t .0 \quad 0 . c \geq 50$ 4.00 C.1 5 C 0.3131$)$ n.tecu C.C?51) $0.00 \quad C .1 \leqslant C \quad C .31 \div 0$ 0.2tEC C. 0.250 $3.00 \quad c .1 \leq C \quad().): 30 \quad 0 .=6 . t<\quad C .(251)$ $10.00 \mathrm{C} .130 \mathrm{C}$ C.3130 0.ted C.L.250 $12.00 \quad 0.150 \quad 0.3130$ J.tec0 0.1235

$2.00 \quad 5.15 C \quad 0.3130 \quad 3.4655 \quad 0.1250$ $4.00 \quad 0.150 \quad 0.21 \% 0$ C.4t54 $\quad .6250$

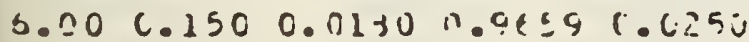

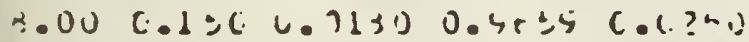

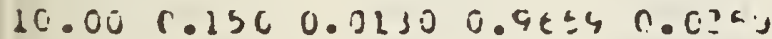
12.0u C.D3C C.313: 0.4cEY C.L:30

$2.00 \quad 0.15 C \quad 0.2130 \quad 0.45 t z \quad .0250$ $4.00 \quad 0.1 \leq C \quad 0.1130 \quad 0.9,9 t .2 \quad 0.1 .25,$.

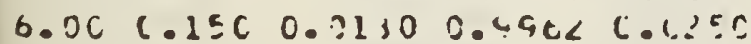

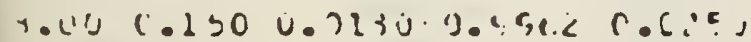

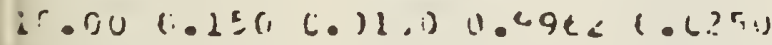

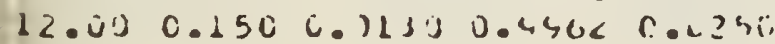

QR: IYI FLL.H

ENTKY JEFTH

SATIO H/L.
ENTEY

ENERGY

m.
NURMAL DEPTH H/O.

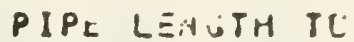
NDEMAL JEPIT. L/D.

$\begin{array}{lll}0.0710 & 0.0406 & 0.1508 \\ u .1124 & 0.1487 & 0.2406 \\ u .1434 & 0.1966 & 0.3240 \\ i .1742 & 0.2385 & 0.3933 \\ u .20<5 & 0.2754 & j .4031 \\ i .2244 & 0.3200 & 0.5300\end{array}$

v. 0577

0.0374

0.1200

4.1400

$0.15<2$

ᄂ. $1=35$

C.051s

$0.000 u$

ن. 2038

0.1251

v. 1447

$0.103 i$

$0.04 \mathrm{dc}$

$(i .07) 1$

ن. 0772

0.1170

$0.13>4$

0.1527

0.0470

0.0720

$0.093 c$

U. 2135

v. 1307

6.2473

6.0405
$0.07 i j$
0.0790
0.121.
0.1223
0.1424
0.1296

0.2149

$0 .<060$

0.3447

$0.400 y$

0.4582

0.1574

0.2636

u. $3=13$

0.4303

0.2025

$0 .>0$ CS

0.1777

0.2905

$0.3 \% 30$

$0.45 \div 2$

0.2097

0.0440

0.1 is

0.3165

0.4259

$0 .=210$

0.0083

0.3844

0.1924

$0.3=23$

0.4334

0.3310

0.0215

0.7032
0.1500

0.2406

0.3146

$0.34 j 3$

0.4 .331

0.5306

0.1548

$0.31>6$

0.3933

0.4631

0.53 J

$0.15 \cup 3$

0.2406

0.3170

J. 3is 3

J. 4031

0.530 is

0.1500

0.2400

$0.31 ; 0$

U.3.153

0.4631

$0.33 u y$

J.1500

$0.24 j 6$

v. $31 \% 6$

J. 3423

U. 4 h 1

0.53 u
0.2406
7. 3323

12.2101

10.U1\%

14. Lovj

21.5713

23.52 .55

7. 7455

13.2053

17.4614

20.35213

$23.04+4$

26. (1) 6

0.2235

13.7321

$10 \cdot 21=8$

21.7415

24.0775

27.2353

6ิ. 3754

14.0502

10.4771

22.?ंखं

23.2 .114

$2 \% .7374$

$6.45=5$

14.1 is

$10 . \sin 33$

$2(0.4 \cdot 8) 1$

$25.400 ?$

दे०.040?

0.4 .73

14.2775

It. 7uis

2<.j.j/3

22.ว4:S

20.130 
FLOK DIA. MANN. SUFPLY CPAIY L/S. Y. COEFF SLCPE SLCPE (SIN) (SIN)
OKA IN FLCH

ENTKY UEPTH

RATIO H/D.
ENTRY

ENERGY

H.
NOKMAL DEPTH

H/D.
PIPE LEIGTH I

NORMAL JEEPTH. L/D.

\section{$2.00 \quad 0.150 \quad 0.01300 .25880 .0167$ \\ $4.00 \quad 0.1500 .3190 \quad 0.25 E E \quad C .(167$ \\ $6.00 \quad 0.150 \quad 0.01300 .2560 \quad 0.4158$ \\ $3.00 \mathrm{C.15C} 0.31300 .25 .600 .4157$ \\ $10.00 \quad 0.150 \quad 0.21 \div 00.256$ e C.C167 \\ $12.00 \quad 0.150 \quad 0.2130 \quad 0.2560 \quad 0.0135$}

\author{
0.0720 \\ 0.1104 \\ 0.1439 \\ 0.1742 \\ W. 2025
}

0.0577

0.0844

0.1160

0.1400

0.1022

i. 1035

0.0518

$2.00 \quad 0.150 \quad 0.131300 .7070 \quad 0.0157$

$4.00 \quad 0.1500 .11300 .7 C 7 C$ C.C157

$6.00 \quad 0.15 C \quad 0.31300 .7070 \quad 0.0137$

$8.00 \quad 0.15 C \quad 0.2130 \quad 0.7070 \quad$ C.4137

$10.00 \quad 0.150 \quad 0.01 .300 .7070 \quad 0.0137$

$12.00 \quad 0.1500 .31300 .7070 \quad 0.0167$

$2.00 \quad 0.150 \quad 0.01300 .8600 \quad 0.0167$

$4.00 \quad 0.15 C \quad 0.2130$ O.tEOC C.4167

$6.000 .15 C \quad 0.21300 .5 t \in 0 \quad 0.0167$

$8.00 \quad 0.1 \leq C \quad 0.21300 .8 .600 \quad C .0167$

$10.00 \mathrm{C.150} 0.01300 .8000 \mathrm{C.C167}$

$12.000 .1500 .31000 .56 t 0 \quad 0.0167$
0.0600

0.1030

0.1251

0.1447

U. $2 \in 32$

0.0486

0.0751

0.0972

0.1170

0.1354

0.1227

0.0470

U.0720

$0.0930^{\circ}$

4.1130

0.1307

0.1473

0.0465

0.0710

0.0030

0.1114

0.12 .33

U. 1459
0.0906

0.1487

0.1466

0.2385

0.2759

0.320 .0

0.1296

0.2149

0.2865

0.3497

0.4009

0.4582

0.1579

0.2636

0.3516

0.4303

$0.2 \cup 28$

0.5635

0.1777

0.2960

0.3940

0.4 e 82

0.2697

0.0445

0.2996

0.3165

0.4259

0.0216

0.0080

0.0194

0.1929

0.3224

0.4334

$0.531 \mathrm{~s}$

0.0216

0.8032
0.1725

0.2766

0.3644

0.4554

0.5300

$0.6146^{\circ}$

0.1725

0.2766

0.3694

0.4530

0.5300

0.6190

0.1725

0.2706

0.3694

0.4558

0.5346

0.6196

0.1725

0.2730

0.3694

0.4558

0.5300

$0.61 \% 0$

0.1725

0.2706

0.3094

0.4520

$0.53=0$

U. 6140

0.1725

0.2766

0.3644

0.4550

0.5356

$0.61>0$
6.5632

10.0312

13.3745

13.317.

12.9317

10.8405

7. 1765

11.6726

14.0255

$10.92=0$

$17.45<0$

$2 y .44$ i4

7.4623

12.1115

15.4874

17.7013

17.7407

20.4605

7.6178

$12.454 . J$

15.0547

10.1373

17.4517

21.03213

2. 6y35

$1<.54: 4$

16.0401

$1=.34=7$

17.0537

21.2435

7. 7174

$12.63 c^{2} 1$

10.11441

16.4503

$10.53 \% \mathrm{~J}$

21.3742 


$$
\begin{aligned}
& \mathrm{D}=0.15 \\
& \mathrm{Q}+22 \mathrm{l} / \mathrm{s}
\end{aligned}
$$


FLDH OIA. MANH.
L\&S. M. CDEFF

SUPPLY CRAIN

SLOPE SLLPE

(SIN) (SIN)
ORAIN FLOW ENTRY

ENTEY UEPTH ENERGY

RATIO AID.
M.
NORMAL OEPTH

H/O.
PIPE LENGTH T

MORALL LEPTH. L/O.

\begin{abstract}
2.00 Q.750 0.00300 .2568 c.05ño
$14.00 \quad 0.25 \mathrm{C} \quad 0.0020 \quad 0.2580 \quad 0.4500$

$16.00 \quad 0.150 \quad 0.0370 \quad 0.25 E 6 \quad$ C. (5.5D

$13.00 \quad 0.150 \quad 0.100+0 \quad 0.25$ te C. .500

$20.00 \quad 0.15 C \quad 0.2070 \quad 0.2566$ C.0500

$22.00 \quad 0.150 \quad 0.3070 \quad 0.2586 \quad 0.0500$

$2.00 \quad 0.15 C \quad 0.0090 \quad 0.5 C C U$ C.C500

$14.00 \quad 0.150 \quad 0.0090 \quad 0.5000 \quad 0.0500$

$16.000 .15(1+0.00900 .5 C C C$ C. 05.00

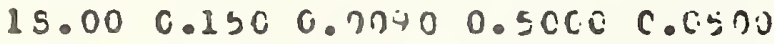

$20.00 \quad 0.15 C \quad 0.39000 .5 C C C \quad C .(.501)$

$22.00 \quad 0.15 C \quad 0.00 \geqslant 00 . E C 00 \quad .0500$
\end{abstract}

$2.00 \quad 0.15 C \quad 0.0070 \quad 0.7 C 70 \quad$ C.CE. 0

$14.00 \quad c .15 C \quad 0.00700 .7070 \quad C .6500$

$16.00 \quad 0.150 \quad 0.00700 .7 C 70 \quad 0.6530$

$18.00 \quad C .150 \quad 0.3230 \quad 0.7 C 7 C \quad C .65 n 0$

$20.00 \quad 0.150 \quad 0.0020 \quad 0.307 C \quad 0.0500$

$22.00 \quad 0.150 \quad 0.2010 \quad 0.707 C \quad r . U 500$

$2.00 \quad 0.150 \quad 0.0070 \quad 0.8 t t c \quad 0.0500$

$14.00 \quad 0.150 \quad 0.0 n 33 \quad 0.8 \in t C$ C.C530

$16.00 \quad C .15 C \quad 0.2032 \quad 0.8 E t U$ C.LSDO

$18.00 \quad 0.1500 .20700 .8 t \in 0 \quad c . c 500$

$20.00 \quad 0.1 \leqslant c \quad c .00700 . t \operatorname{tec} c . c 5 n 0$

$22.00 \quad 0.15 C \quad 0.7040 \quad 0 . E t, t C \quad$ C.OSnO

$2.00 \quad 0.250 \quad 0.00730 .9 t 590.0500$

$14.00 \quad 0.15 C \quad 0.3770 \quad 0.5 t 59 \quad 0.0500$

$16.00 \quad 0.150 \quad 0.3070 \quad 0.9659 \quad 0.0500$

$18.00 \quad 0.150 \quad 0.01700 .4654 \quad C . C \equiv C 0$

$20.00 \quad 0.15 C \quad 0.2070 \quad 0.565 .4 \quad 0.01500$

$22.00 \quad 0.150 \quad 0.0070 \quad 0.9654 \quad 0.0500$

$2.00 \quad 0.150 \quad 0.6030 \quad 0.99 t 2 \quad 0.0500$ $14.00 \quad c .1 \div 0 \quad 0.3010 \quad 0.49 t \angle C .0 .03$ $16.00 \quad c .150$ c.0.10 0.4902 C.C5no $19.00 \mathrm{C.130} 0.02100 .44 t<\mathrm{C} .0500$ 20.000 .150 C.3040 n.496: C.C.500

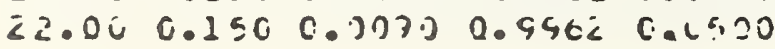

0.0460
0.1543
0.1742
0.1256
0.2025
0.2102

0.0375

0.1233

0.1400

0.1515

0.1622

0.1730

0.0337

0.1247

0.1251

0.1351

0.1447

0.1542

0.0310

0.1674

0.1170

0.1203

0.1324

0.1442

0.0306

0.1037

0.1130

$0.122 \mathrm{~V}$

0.1307

0.1340

0.0303

0.1020

0.1119

U. $120 \mathrm{E}$

0.1243

0.1376

\subsection{3 \\ 0.3015 \\ 0.8755 \\ 0.9454 \\ 1.0123 \\ 1.0750}

0.2914

1.2181

1.3357

1.4441

1.3545

1.0546

0.3592

1.5175

1.0051

1.3066

1.9463

2.0731

0.4069

1.7281

1.9002

2.0623

$2: 2177$

2.3662

0.4344

1.0506

2.0363

2.2045

2.3757

2.2415

0.4426

1. 0499

2.0754

$2 .<539$

2.4292

2.3953

\subsection{0 \\ 0.2791 \\ 0.3064 \\ 0.3333 \\ 0.3596 \\ 0.3850 .}

0.0770

0.2791

0.3004

0.3333

0.3596

0.3050

0.0770

0.2791

0.3004

0.3313

0.3546

0 . 3 3 50

0.0770

$0.27 \times 1$

0.3004

0.3333

0.3596

$0.38>0$

0.0770

0.2741

0.3004

v. 3333

0.3590

$0.30>0$

0.0770

0.2791

0.3004

0.3333

0.3540

$0.39>0$
17.4232

76.2177

$83.20 . j 4$

94.6257

90.14 .7

$103.71 \neq 0$

19.4003

$85.057 \%$

42.9312

101.1729

104.5372

$11 \% .8032$

20.2537

88.4000

90.6075

105.3027

113.4653

120.6412

20.0617

90.2155

90.6440

107.4270

210.2045

123.0747

20.0545

$91.06 \%)$

94.5133

$100.452 \%$

$117.313 \div 1$

$124.2+33$

20.9075

91.3227

9). 0503

105.7110

$217.04=7$

124.6471 
FLOH OIA. MANN. LIS. H. COEFF
SUPPLY DFAIN

SLCPE SLIOE

(SIN) (SIN)
DRAIN FLOH

ENTRY OEPTH ENERGY

RATIO HIO. H.
NURMAL DEPTM

H/D.
PIPL LE.TGTH TO

MORAAL JEPTH. LIU. $\begin{array}{lllll}2.00 & 0.15 C & 0.0390 & 0.2588 & 0.0137 \\ 14.00 & 0.15 C & 0.1010 & 0.2565 & 0.0157 \\ 16.00 & 0.15 C & 0.1020 & 0.2586 & 0.01157 \\ 19.00 & 0.150 & 0.3970 & 0.2586 & 0.0 .147 \\ 20.00 & 0.15 C & 0.10 .10 & 0.25814 & 0.1137 \\ 22.00 & 0.150 & 0.0090 & 0.2588 & 0.0157\end{array}$

$2.00 \quad 0.150 \quad 0.3030 \quad 0.5 C C C \quad 0.0167$ $14.00 \quad 0.150 \quad 0.20 .730 .5 c c 0 \quad 0.0157$

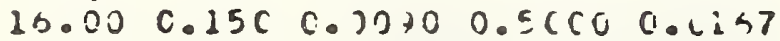

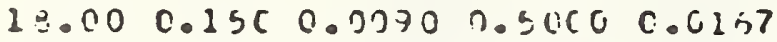
$20.00 \quad C .1=C \quad 0.13300 .5 \operatorname{ccc} C . C 14.7$ $22.00 \quad 0.150 \quad 0.190700 .5 C C O \quad 0.6267$

$2.00 \quad 0.150 \quad 0.00100 .707 C \quad 0.0167$ $14.00 \mathrm{C.150} C .39 .00 .7 C 7 \mathrm{C}$ C.C147 $16.00 \mathrm{C.SSC}(1.30300 .7 C 7 \mathrm{C}$ C. 0167 $18.00 \quad 0.150 \quad C .3030 \quad 2.7 C 7 C \quad$ C. 1157 $20.00 \quad 0.1 \leq C \quad 0.33 \geqslant 0.7 C 7 C .0 .0137$ $22.00 \quad 0.150 \quad 0.73700 .7570$ C.C167
2.0J $\quad 0.156 \quad 0.3230 \quad 0.9554 \quad 0.51 \leq 7$

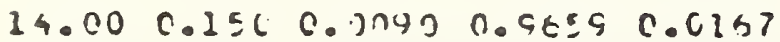

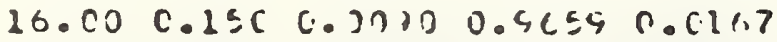

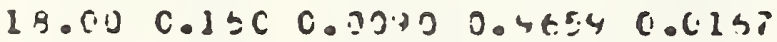

$20.000 .150 \quad 0.17100 .5455$ C.C147

$22.00 \quad 0.150 \quad 0.3070 \quad 0.5659 \quad 0.4157$

$2.000 .15 C \quad 0.02900 .99420 .0157$

$14.00 \quad 0.1500 .3010$ C.598.4 C.C137

$16.00 \quad 0.156 \quad 0.35109 .4962 \quad 0 . C 1$ in

$10.00 \quad C .1 \leq C \quad 0.1)$. $0.496<$ (.0:6?

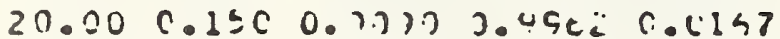

$22.000 .150 \quad 0.03300 .496 \overline{0} 0.0 .147$
0.0460
$0.15+3$
0.1742
$0.13 r, 6$
0.2025
0.2102

0.0375

0.1203

0.1400

0.1513

0.1022

0.1730

0.0337

0.1147

0.1252

0.1351

0.1447

0.1542

0.0326

0.1074

C. 1170

0.1203

0.1354

C. 1442

0.0305
0.1037

4.1130

$0.122 \mathrm{C}$

0.1307

$0.13 \times 0$
0.1973

0.0015

0.0755

0.4454

1.0123

1.0750

0.2919

1.2181

1. 3357

1.3545

1.6546

0.3592

2.5175

2. cots 1

1.0066

1. 4463

2.0731

$0.40 t C_{1}$

1.7281

1. 9002

2.002 .5

2.2177

2.3062

0.4344

1.0500

2.0363

2.2096

2.3757

2.5415

0.0303

0.1026

0.1119

U.120

C. 0.13

0.2310

0.4426

1.8894

$2.075 \%$

2.0533

2.4292

$2.29 \leq 3$
1.4441

0.1093

0.4233

0.4558

0.4976

0.5306

0.5716

0.1093

0.4133

0.4538

0.4476

$0.53=6$

$0.57 \% 0$

0.1093

0.4133

$0.4 j 56$

0.4976

0.5306

$0.57 \times 6$

0.1093

0.4133

0.4550

0.4776

$0.53=6$

$0.57 \times 6$

0.1043

0.4133

0.4598

0.4976

0.5306

0.5740
0.2093

0.4233

0.4550

U.4 136

$0.53=0$

$0.57>0$
28.3700

$117.603 j$

127.4747

$136.27 \% 7$

$143 \cdot 14=0$

155.5575

29.9749

124.7203

139.3733

$14=.5=12$

152.20 .3

$165.53 . j$

30.6478

127.5335

130.7570

144.3213

154.2410

$16.941 y$

31. 30.59

129.4513

14 . 5025

151.3127

161.3575

170. $\varepsilon c<0$

$31 \cdot 1=50$

230.2003

143.4414

154.5309

104.7503

107.5237

31.2345

124.437 .0

143.1702

15\%.11\%:

$160 \cdot 13,3$

100.0!: 1 


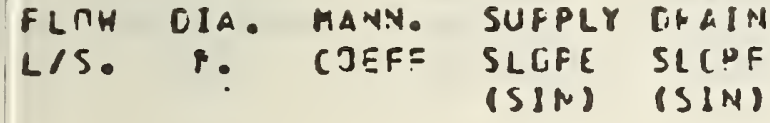

$2.00 \quad 0.19 C \quad 0.3990 \quad 0.258 t \quad 0 . C 17.5$ $14.00 \quad 0.150 \quad 0.37100 .2360 \quad c .4125$ $13.00 \quad C .1 \leq C \quad 0.2730 \quad 3.2586 \quad 0.1 .125$

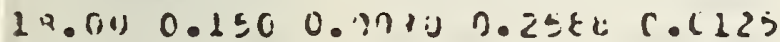
$20.0 u$ C. $13(0) .0100.25 \varepsilon 0$ C.ul? $22.000 .130 \quad 0.33700 .25 t 0 \quad 0.0125$

$2.00 \quad 0.25 C \quad 0.30 .700 .5 C 0 C$ C.C12.5

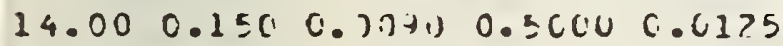

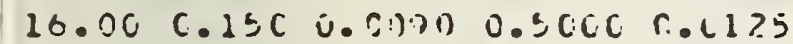
$16.00(.150(1.7)) \cup 0.50 e c(.0 .125$

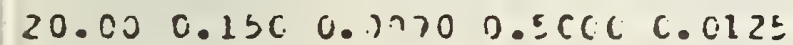
$22.00 \quad 0.15 C \quad 0.3 .770 \quad 0.5(C 0 \quad 0.125$

$2.00 \mathrm{C.15C} 0.30300 .7070 \quad 0.0125$ $14.0 \cup \quad C .1 \leq 0$ i. $03100.7 C 70 \quad$ c. (i125 $10.00 \quad C .1 \leq C \quad 0.30 \neq 0 \quad 0.7 C 7 C \quad C .0175$ $1.4 .00 \quad 0.1 \leq 0 \quad 0.1 .7100 .70700 .0125$ $20.000 .150 \quad 0.0 .0100 .7 C 70 \quad 0.0125$

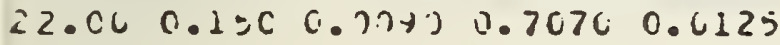

$2.000 .1 \leq C \quad 0.3070 \quad 0 . \varepsilon+t C .0 .0125$

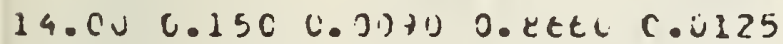

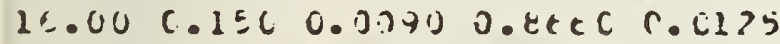
$1 \therefore .00 \quad 0.15 C \quad 0.3970 \quad 0 . t+t C \quad c .0125$ $2 J .0 C(.1: C(0)) ;$.0 U.e CCC C.C1 125

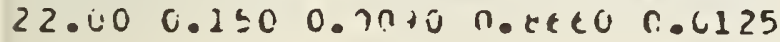

$2.00 \quad 0.150 \quad 0.0010 \quad 0.9654 \quad 0.0125$ $14.000 .15 C$ C.. $001 j 0.9 t 590.0123$ $16.00 \quad(.15 C \quad 0.503 C \quad 0.9 t \leq 9 \quad$ C.C125 $14.000 .156 \quad 0.30700 .94: 5$ C.L125

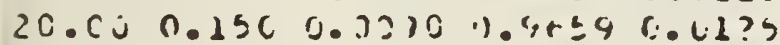
$22.00 \mathrm{C.13(0.1)2)0} 0.4654$ (.6.)?

2.00 .1360 .30100 .996 C.CIPS $14.00 \mathrm{C.13C}$ C. 0.2170 .54 te C.L125 le.cu $0.1360 .9 n>0$ n.498.2 C.0125

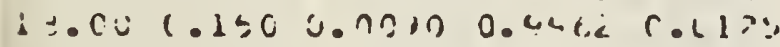

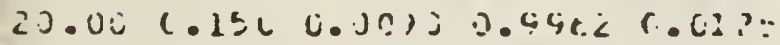

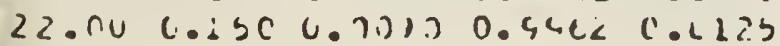

DRAIN FLOH ENTRY

EHTKY CEEPTH ENEREY

RATIU H/O. M.
MURHAL DEPTH H/D.
PIPE LEIVTH TC NURMAL JEPTH. L/U.

$\begin{array}{llll}0.0460 & 0.1973 & 0.1201 & 30.8745 \\ 0.1543 & 0.3615 & 0.4547 & 124.77 c 7 \\ 0.1742 & 0.0753 & 0.5033 & 130.1553 \\ 0.1936 & 0.7454 & 0.5522 & 145.0017 \\ 0.2025 & 1.0123 & 0.6021 & 124.5150 \\ 0.2162 & 1.0750 & 0.6479 & 162.4001\end{array}$

0.0375

0.1253

U. 1400

0.1315

$0.16<2$

0.1730

0.0337

6.1147

0.1251

0.1351

0.1447

0.1542

c. 0316

U. 1074

$0.117 \overline{0}$

0.1263

$0.13 \% 54$

0.1442

0.0300

0.1037

0.1130

0.1220

ט.1307

0.23 .00

0.0303

0.1020

U.1114

U. $12 \mathrm{JH}$

ט.12.1

6.1376
0.2919

1.2181

1.3357

1.4441

1.3545

$1 \cdot 0546$

0.3592

1.2175

1.0651

1. 3c05

1.4463

2.0731

0.4069

1.7201

1.9002

2.1025

$2 . \angle 177$

2.3062

0.4344

1.0503

2.0263

$2 .<09 n$

2.3757

2.3415

0.4420

1.0044

2.0749

L.253!

$2.4 ? 92$

$2 \cdot \because 4>3$
0.1201

0.4547

0.5532

0.0021

0.3474

$0.45+7$

0.5003

0.5522

$0.60: 2$

$0.647 y$

0.1201

0.4247

0.5303

0.5522

U. 0021

0.6419

0.1201

0.4547

$0.50=3$

0.5302

$j . \approx 0<1$

J.6474

0.1201

ن. $45 y$ ?

ن. 5053

$0.55><$

$0.0 \dot{0}<1$

i. 6.484
0.3003

0.1201
32.4442

$23 i .3574$

141. ibso

153.53\%2

$10, .1921$

$17 \mathrm{c} .3004$

33. 1243 137.350 נ 140.3204 169.1700 $164.100 \mathrm{~J}$ 171.64 .34

$33.47,3$ $150.57: 2$ $145.14 \mathrm{~J}$ $17 b .9 .144$ $10 \mathrm{C} \cdot+4=7$ $16 / .05: 5$

\subsection{2}

100.1705

$144.53: 7$

180.4121

$154.32: 7$

$165.5:=7$

33.7033

172 . ves 4

144.jh:?

100.513

1ว

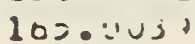


FLOW CIA. MAN.Y SUPPLY ORLIN LIS. H. CDEFF SLCPE SLUPE (SIN) (SIN)
ORAIN FLOW ENTKY DEPTH RAT $10 \mathrm{H} / \mathrm{D}$.

\section{ENTRY \\ ENERCY \\ M.}

NORHAL DEPTH H/O.
PIPE LEIGTH TO MOKMAL DEPTH. LID.

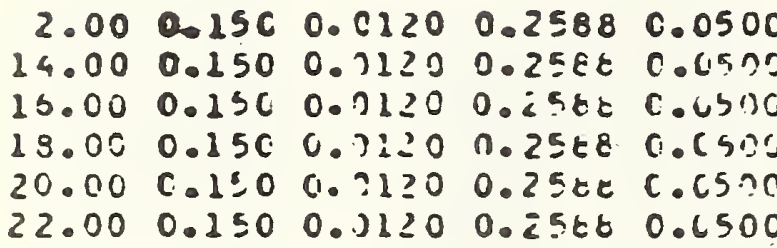

$2.00 \quad 0.15 C \quad 0.3120 \quad 0.5 \operatorname{scc} 0 . \operatorname{cono.}$ $14.00 \quad c .15 C \quad c .3120 \quad 0.5 C C O \quad C . C 500$ $16.00 \quad 0.1500 .31200 .5 C(C) C .0 \leq n c$ $1.2 .00 \quad 0.150 \quad 0.3120 \quad 0.5 c c c c .05 n 0$ $20.00 \mathrm{C.1SC} C .01100 .50 \mathrm{CCC}$ C.0.5 20 $22.00 \quad 0.150 \quad 0.31200 .50 C 00.6500$

$2.00 \quad 0.15 C \quad 0.01200 .7 C 7 C \quad 0.0500$ $14.00(.150 \quad 0.31200 .7070 \quad c . c 530$ 10.000 .1560 .01220 .76700 .0500 $18.00 \quad(.15 C \quad 0.11 \geq 0 \quad 0.7 C 70$ C. 6590 $20.00 \quad 0.15 C \quad 0.012 .00 .7 C 7 C \quad C . C 5.00$ $22.00 \quad 0.150 \quad 0.21200 .7 C 7 C \quad 0.6500$

$2.00 \quad 0.15 C \quad 0.0120 \quad 0 . t C \in C \quad 0 . C 500$ $14.00 \quad C .1 \leq C \quad 0.31 \geq 0$ D.tetco C.0in $16.00 \quad c .15 C(0.1) 1 \geq 0 \quad 0 . \varepsilon \in t 0 \quad c . c 530$ $18.100 \mathrm{C} .15 \mathrm{C} \quad 0.31200 . \varepsilon t e . C .6 \div 20$ 20.JC C.15C $0.01200 . E \in t C \quad 0.0 .500$

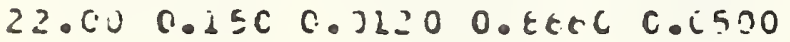

$2.0150 .156 \quad 0.5120 \quad 0.5459$ ci.c500

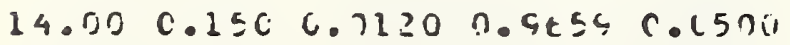
$16.00 \quad 0.1500 .1120$ 0.465S (.CE 00 $18.00 \quad 0.150 \quad 0.31200 .96 \leqslant 5$ C.C5.01 $20.00 \quad c .150 \quad 0.11200 .9259$ c.t5no $22.000 .15 C \quad c .11200 .06 \leq 90.6530$

$2.000 .1560 .0120 \quad 0.99020 .0500$ $14.00 \mathrm{C.25C} 0.31200 .99(2 \mathrm{C.Uj}: 3$ $13.30 \quad 0.1 \leq C \quad 0.11200 .9 c t z \quad 0.050 j$

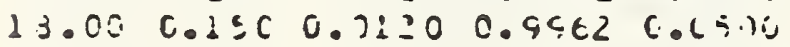
$20.00 \quad 0.1 \leq c \quad c .0 .1=0 \quad 0.9 c e<c . c b 00$

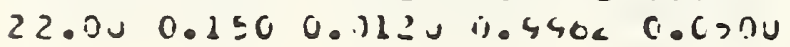
0.0550
0.19 .32
0.2115
0.2294
0.2458
0.2634

\begin{abstract}
0.0440
0.1522

0.1640

0.1835

0.1769

0. $21 \cup 1$
\end{abstract}

$0.04 ن 3$

$0.13=3$

0.1510

4.1032

0.1752

ن. 1369

0.0378

0.1215

0.1412

0.1527

0.2637

0.1744
0.1413

0.5374

0.0077

0.6544

0.4980

0.7417

0.2071

0.0430

0.4219

0.9966

1.0683

1.1350

0.2546

1.0523

1.1530

1.2441

1.3387

1.4232

0.2873

1.2958

1. 3133

1.4217

1.5274

1.0276

0.3073

1.2841

1.4067

1.2247

1.0402

1.7444

i. 0362

0.1237

0.1349

U.145\%

0.1204

0.1000
0.3123

1. 3090

1.4368

1.2545

1.6704

1.7001
0.0925

0.3420

0.3767

0.4104

$0.443 n$

0.4758

0.0925

0.3420

0.3707

0.4104

0.4436

0.4750

0.0925

$0.34<0$

0.3707

0.4104

0.4436

0.4758

0.0925

0.3420

0.3707

0.4104

0.4436

U. 4758

$0.09 \angle 5$

0.3420

0.3707

0.4104

0.4436

0.4755

0.0925

0.3420

0.3707

0.4104

0.4490

0.4725
11.8880

50.3711

50.3580

54.771 J

$64.24 \times 4$

$67.327 j$

19.2442

50.1631

01.6304

6t. $03 \mathrm{~J}$

71.0370

75.0002

$13.77>1$

50.40נ0

$64.27=0$

64.4310

$74.025 j$

70.20052

14.0445

59.3304

$02.50=0$

$70 \cdot 54 ? 4$

$70 \cdot: t=2$

$80.53>7$

14.1313

00. 2411

00.1430

71.2543

70.7440

01.3473

14.2235

OU. 4005

$00.3+11$

71.7410

77.1373

01.2740 


\section{FLOH CIA. L/S. M. \\ MAYN. \\ C.JEFF \\ SUPPLY CFAIN \\ SLIIFE SL:PE \\ (SIN) (SI.V)}

OKi IN FLOH

ENTKY UFFTH

RATIO HID.

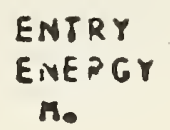

NURMAL UEPTH

$H / O$.
PIPL LETITH TL NOKAAL JEPTH. L/D.

\begin{abstract}
$2.00 \quad 0.150 \quad 0.0120 \quad 0.2568 \quad 0.0250$ $14.00 \quad 0.150 \quad 0.01200 .256 C \quad$ C.C25C $16.00 \quad 0.1500 .3120 \quad 0.25 .64 \quad$ C. $18.00 \quad c .150 \quad 0.1120 \quad 0.256 t \quad C .1250$

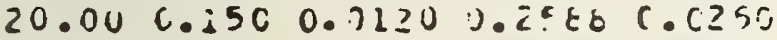
$22.00 \quad 0.150 \quad 0.1120 \quad 0.25 t 5 \quad 0.0250$
\end{abstract}

$2.00 \quad 0.150 \quad 0.01200 .5000 \quad 0.0250$. $14.00 \quad 0.150 \quad 0.3120 \quad 0.50 C u \quad 0.0255$ $16.000 .1300 .01200 .5 r(i c c . i 251)$ $19.0 \mathrm{C} C .1500 .31: 1) 0.5 \mathrm{CCC} C .6250$ $20.00 \quad 0.150 \quad 0.31200 .5 C(C \quad$ (.. 2550 $22.00 \quad C .1=C \quad 0.01200 .5 . C L C \quad C .0250$

$2.00 \quad c .150 \quad 0.31200 .7 C 70 \quad C .0 .250$ $14.00 \mathrm{c} .150 \mathrm{C} 0.31230 .70 .7$ u $(.02550$ $16.00 \quad C .150 \quad 0.01 .200 .76 .76 \quad 0.0250$ $13.00 \quad c .1500 .11200 .707$ U l..4250 $20.00 \quad 0.150 \quad 0.0120 \quad 13.7(.7 C \quad$ r.c.c25u $22.00 \quad 6.150 \quad 0.2120 \quad 0.707 i \quad c . c 2 \div 0$

$2.0 C \quad C .15 C \quad 0.3120 \quad 0 . \varepsilon \in \in C \quad C .0 .250$ $14.00 \mathrm{C} .1 \mathrm{bC} 0.31 \geq 0)$ 1). PEtC C.C25: $16.00 \quad(.1) C \quad 0 . C 1 \geq 0 \quad 0 . \varepsilon+t C \quad C . C 2=0$

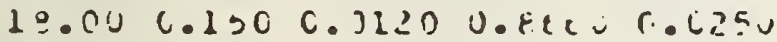

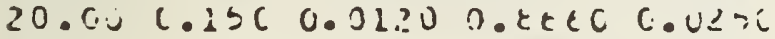
22.00 C.:SC 0.3120 o.st.tL C.U25u

$2.00 \quad 5.25 C \quad 0.21230 .5654 \quad 8.025,1)$ $14.00 \quad \therefore 15 C \quad 0.0120 \quad 0.0 .59 \quad C .1250$

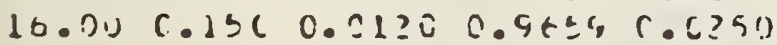

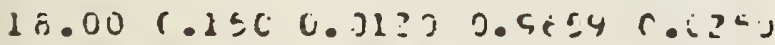
$20.0 .0 \quad 0 . i \leqslant c \quad 0.31 \geq J \quad J .4+24 \quad c . i \geq 5.0$ 22 . ou C.1SC 0.J120 0.citsy C.uibu

$2.00 \quad 0.1 \leq C \quad 0.21 \geq 0 \quad 0.55 t 2 \quad 0 .(200$ $14.000 .1360 .71 \geq 00.1,48.21 .(2 a$, $15.00 \quad 0.250 \quad 0.3120 \quad 0.4982 \quad 6.025 .1$

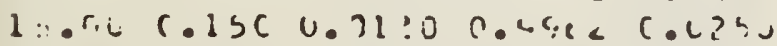
$20.0 \mathrm{U} C .15 \mathrm{C}$ U. $1120 \mathrm{~J}$. GGe $1.0 . \mathrm{G}$

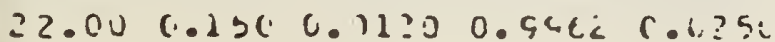

0.0550
$u .1712$
$u .2115$
0.2244
0.2403
0.2034

0.0446

0.1552

L. 1590

v. 1135

ن. 1909

0.2101

0.0403

L. 1323

0.1510

U. 1632

c. 1732

0.1309

$0.037 t$

U. 12.95

U. 1412

0.22 .27

u. 153 ?

0.1744

0.0365

ن. 1244

0.1304

0.1473

i. 1270

$0.160 j$

C. 0352

0.123 ?

i. 1344

‥14

i. 1204

c.l一ot.
0.1413

0.3574

0.6077

0.0544

0.0980

0.7417

0.2071

0.0430

0.721 .7

0.4305

1. 0653

$1.135 b$

0.2546

$1.0=23$

1. 1530

1. $<441$

1. 35 ह 7

1.4232

0.2673

1.1950

1.1133

1.4217

1.2274

$1.0<70$

0.3073

1.2441

1. 4007

1.3247

1.0402

$1.744 t$

0.3123

1.304 .5

1.4300

1. $2: 4$.

1.. 7204

1.1201
0.1155

$0.44 \cup 2$

0.4501

0.5300

.0 .5757

0.0107

0.1155

0.44 is 2

0.4961

$0.53 \cup 8$

ग. 5757

ט.6137

0.1125

0.4402

0.4501

0.5308

0.5757

0.6157

0.1155

0.44 .2

U. 4 हU 1

0.5108

$0.57=7$

0.0167

0.1155

0.4402

$0.4 \div 01$

0.5303

0.5757

U.61, 7

0.1135

0.4402

v.4801

U.5ju=

ن. $7>7$

0.0107
15.7523

44.334 ?

69. 잔ㄷ?

74.2447

EU. 12,3

83.0744

10.9137

64. 2411

75. 32,5

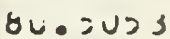

60.5 520

บ以・ンつ 7

17.4027

71.4273

71.7 .45

89.134t

c.8.34,

92.524

17.0244

$7_{c}=7_{j}$

7c.

d4. 4 13J

4u: $=i+4$

पy.

17.72 .3

73.570

74. 79 4

$\forall=1 ; 03$

Y $10.57 \mathrm{~s}$

†ว . 160 1

17.120

73.3203

$77.70 ; 5$

c). $5 \% 3$

Y 10 . 70.

$40.2 \% 1$ 


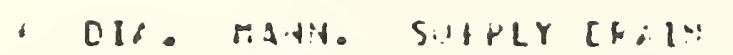
P. CJFF SLINF SL: DE $(5 ! 1) \quad,(5,2)$

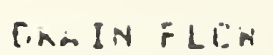

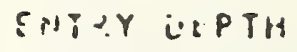
"LTiis ali.
Eirthit

Eiv̈̌GY

14.
NU2MLL UEPTI H/D.
PIPC LEVITH Y:

NUK.FIL $J: P T: 1$. L/ii.
30 $0.15($ c.71.2) ().

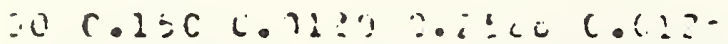

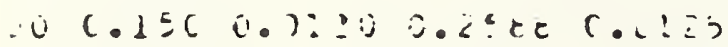

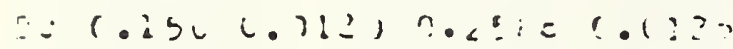

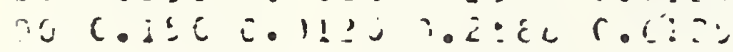

Ju

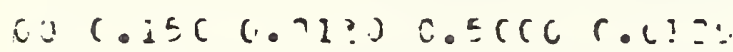

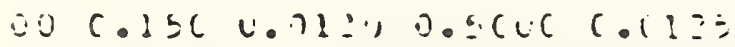

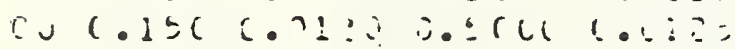

$\because$ C.

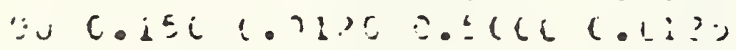

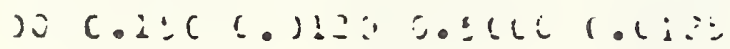

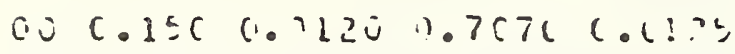

こ C.IË

uj C..

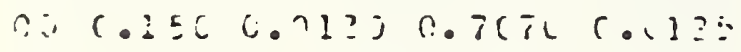

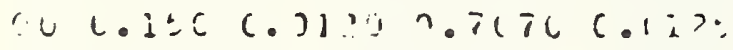

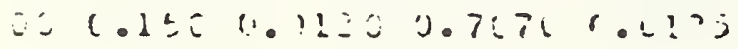

$200.156 \quad 0.91200 .8 .+6$ C $1.12: 5$

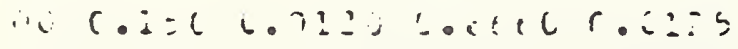

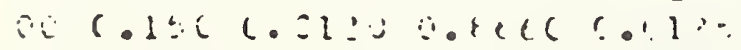

.

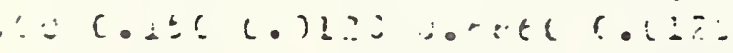

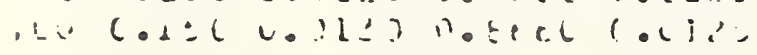

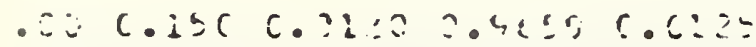

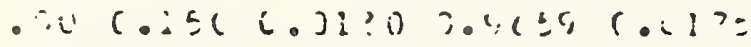

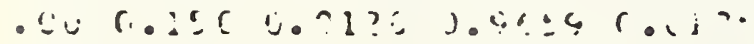

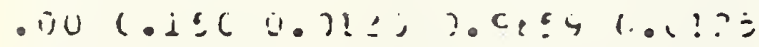

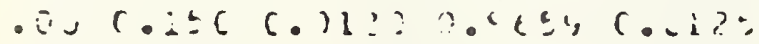

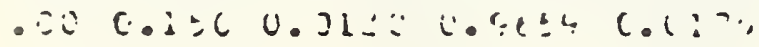

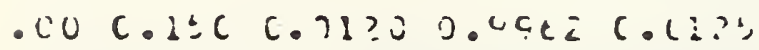

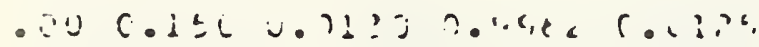

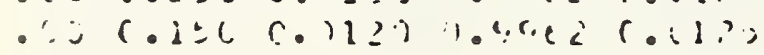

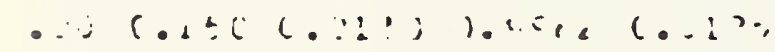

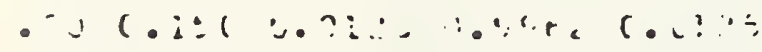

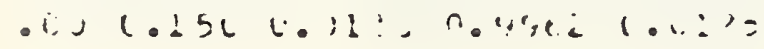

\begin{abstract}
$0.0,550$
4.1252

L. $21+3$

$6.22 \div 4$

$\therefore .243$.

v. 2034
\end{abstract}

C. $344 i$

U. 1\%:

6. ? = =

w. : $=35$

L. 1 ic 7

u. 2isl

5. $1: 4: ; 3$

(c. $0.13=3$

L.25iJ

C. $1 \div 92$

i. 1752

ᄂ.1..

ن. $337=$

j. 12.5

ᄂ.1 $14: 2$

$0.1: 67$

i. $10 \div 7$

i. 1 i 44

$0.0,3: 5$

u. 124.1

$u \cdot 13: 34$

L. 1473

U. $1,7=$

;.10,3

i. $23=i$

U. 123

1.. 1394

य. is 9.

v. $i^{-1}-4$

(.) 1 ":os,
0.1413

0.5274

0.0077

$0 . \therefore, 44$

$0 . \because 28: ?$

0.7417

0.2071

0.0430

0.9214

0.730 .0

1. vi.te 3

1. .355

$0 .<: 44$

1. $J=23$

1. 133

1. 24.1

1. $3 \overline{3.7}$

1. $4 \overline{2} ; 2$

2. 2c 73

1.655

1. $9: 33$

1.4617

$10 ; 274$

1.0 .75

$0.2: 73$

1... 41

1. + +10.7

$1.0<47$

1. $: 402$

1. 1447

0.

1.

1.426;

1., $14=$

$\therefore .704$

1. 7.51
0.1449

ग. $27: 3 x$

ט.0.

$3.51: 4$

נ. $1 \div: 4$

J. 8120

0. 1447

U. $27 \cup \mathrm{C}$

j. 03.3

j. $=t<4$

$0.7=94$

U. $=16 j$

v. $144 y$

ग. 27.03

J.53

j.

j. 7, 44

נ. $31: 0$

0.1449

ن. 574 o

$0.63: 3$

0.5927

$3.7 \div 34$

j. $31=0$

0.1449

j.57

0.0363

v.t.

ن. $7:=4$

ग. $-1<0$

U. 1443

3.570.

$0.03<3$

0.5y-

ง. $7 \cdot 04$

i. $=125$
$12.107,1$

6. 47,3

$76.71=0$

$74 .: 001$

77 . 74.4 .40

00.4315

14. icol

$7 \therefore 3=02$

7c. $\vdots .12$

iv. 3', ?

(4.3-3

67. 713

17.0.45

$77 . j 2=3$

=6. 34 :

$04.31: 4$

oc. 30,4

Gu. ijs 2

14.

$=4 .: \div 2$ ?

ن4. 活,

こن.je:?

(.) $7 \ldots 3$

i $i_{6} . .2 i 2$

L.: : : j

$81.53+0$

ce.tid

5y. 53 ,

-7.0s,

16:. : : 1

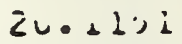

¿: $47:$ :

ㄴ..

'u. Ei)

ci. $74, \mathrm{~J}$

Lu... ? . ! 
FLOH CIA. L/S.
H.

MAVN CJEFF
SUPPLY TKALI

SLCPE SLUFE

(SIN) (SDN)
URA IN FLOH

ENTRY OEPTH

RATIO H/L.
ENTRY

ENERGY

n.
MORTAL UEPTH H/O.
PIPL LE.NGTH TO NORMAL JEPTH. L/U.
$2.000 .1500 .01200 .25 \varepsilon t \quad 0.0167$ $14.00 \quad 0.150 \quad 0.1120 \quad 0.25 t$ C.C167 $16.00 \quad 0.150 \quad 0.21 ? 0 \quad 0.25+\varepsilon \quad C .1 .1 n 7$

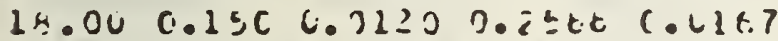
$20.006 .156 \quad 0.11200 .2563 \quad 0.014 .7$ 22.040 .1500 .91200 .2560 C.U1h?

$2.000 .250 \quad 0.91200 .5 \operatorname{ccc} 0.0167$ $14.00 \quad 0.150 \quad 0.21200 .5 C C C \quad 0.0147$ $16.00(.150 \quad 0.0) 1200.5 C(0) . C 1 F, 7$ $18.00 \quad 0.25 C \quad 0.12200 .5 C C L C . C 167$ $20.000 .1500 .01200 .5 \mathrm{Ccc} C . \mathrm{Cl} 137$ $22.00 \quad 0.150 \quad 0.31 \geq 0 \quad 0 . \pm C . C \quad c .1157$

2.cJ C.15C 0.0120 0.707C C.4167 14. . $0.1500 .11200 .767 c$ r. 0.167 $16.00 \quad(.150 \quad 0.120 \quad 0.76 .7 \mathrm{C} \quad . .(157$

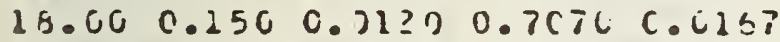
$20.00 \quad 5.15 C$ C. $61 \geq 0 \quad 0.7 C 7 C \quad .6157$ $22.000 .150 \quad 0.01280 .7070 \quad 0.0 .147$

$2.000 .15 C \quad 0.01 \geq 0.0 . E \in E L \quad C .6167$ $14.00 \quad 0.1500 .11 \geq 0$ 0.fete $(.0147$ $16.00 \mathrm{C} .1 \leq \mathrm{C}$ C. . 120 o.tet.0 [. $[16]$

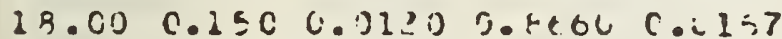
20.00 0.15C i. :120 O. etc.c C.L167 $22.006 .150 .0 .3200 . \operatorname{tec} 0.0157$

2.000 .1500 .01100 .56 .54 C. 1.167

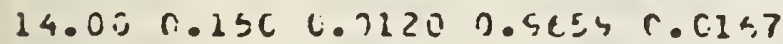
$16.00 \quad C .150$ 0.0:20 0.5.5 C.C. 157

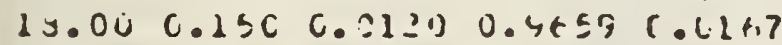

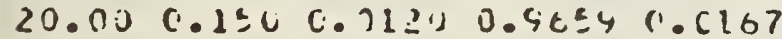
$22.00 \mathrm{C.ISC} 0.1) !: 02.56 .5 \mathrm{r} .1107$ $14.00 \quad 0.13 C \quad 0.11 ? 0 \quad 0.9$. SE $C .0167$ $16.00 \mathrm{C} .15 \mathrm{C} 0.01200 .54 t 26.0157$

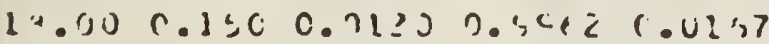

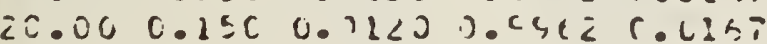
$22.040 .1=0 \quad 0.31 \geq 0 \quad 0.59<6.01 \pm 7$

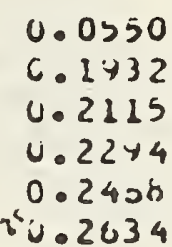

0.0448

0.1552

G. $16+6$

ن. 1835

4.1709

0.2101

0.0403

0. $13 \div 3$

c. 1510

0.1632

U. 1752

v. $1 \div 09$

C. $037 d$

c. $12: 55$

0.1412

U. $13<7$

v. 1537

0. 1744

0.0355

0.1244

c. 1304

i. 1473

U. $157 t$

U. 1603

U. 0362

U. 1237

u. $134^{\prime} ;$

$0.14 j t$

j. $15: 4$

ง. 1000
0.1413
0.2574
0.6077
0.0544
0.6460
0.7417

0.2071

0.0430

0.5219

0.4465

1. 0603

1. 135 t

0.2546

1. 0523

$1.1=30$

1. 44 ? 1

1. 3387

1. $4 \overline{2} 32$

0. $\angle \approx 73$

.1 .1953

1. 3133

1.4217

i. 5274

1.0270

C. 3073

1. $2 \pm 41$

1.4067

1. 2247

1.0492

1. 7449

0.3123

1. 3690

1. 4300

1.j24,

1. $0,7: 74$

1. 7001
0.1320

0.5112

U. 5659

ن. 6140

0.6724

0.7241 .

$0.23<0$

0.5112

0.5659

0.6196

0.6724

J. 7241

$0.13<0$

0.5112

U. $565 y$

$0.01>6$

0.6724

0.7241

0.1320

0.51:2

0.5659

$0.31>6$

$0.07<4$

0.7241

0.1320

0.5112

U. 5659

u. 0140

v. 6124

0.7241

$0.13<0$

0.5112

v. $56=4$

U.61,

J. 0724

0.7241
17.7315

67.7004

73.0213

$70.140 J$

$8<.3 \backsim 5 j$

o5. 9727

16.0156

72.5074

7 c.57

63.9702

bc. 7111

$9<.7134$

14. 2605

$74.73+3$

ชט.77. 3

$80 \cdot 6,11$

41.5110

45.7534

19.5 303

75. 7 ว

84. ¿̇s

$80.4=3$

$91: 707 v$

$24 \cdot 1 \mathrm{i} i 7$

14.6543

$7 \% .24: 3$

$91.304 J$

80.144

4 i. $<7$ g

9ט.3 7 s.

$19.6 \div 3$

77.7 is

4..化s

ะง.77;

41.i., 3

84.3404 
FLOM DIA. MAYH. L/S. H. COEFF
SUFPLY DRAIN

SLCPE SLCPE

(SIN) (SIN)
DRAIN FLQH

ENTRYY UEPTH

RAIIO HIO.
ENTRY

ENERGY

M.
NORMAL

DEPTH

H/D.
PIPE LENGTH

NORMAL JEPIr L/J.

$\begin{array}{lllll}2.00 & 0.150 & 0.3150 & 0.2588 & 0.0500 \\ 14.00 & 0.150 & 0.0150 & 0.2586 & 0.0500 \\ 16.00 & 0.150 & 0.0150 & 0.2568 & 0.05 .00 \\ 16.00 & 0.150 & 0.1120 & 0.2548 & 0.0500 \\ 20.00 & 0.150 & 0.0150 & 0.2580 & 0.0500 \\ 22.00 & 0.150 & 0.12120 & 0.2580 & 0.0500\end{array}$

$2.00 \quad 0.150 \quad 0.0150 \quad 0.5 c 600.0500$ $14.00 \quad 0.150$ C. $0.31500 .50 C C \quad 0.0 .500$ $16.00 \quad 0.150 \quad 0.1250 \quad 0.5$ Ci..0 C. C500 $13.00 \quad c .150 \quad 0.3150 \quad 0.5(100 \quad 0.0500$ $20.000 .1500 .01500 .5000 \quad 0.0500$ $22.00 \quad(.150 \quad 0.2) 1500.500 C \quad c .0500$

\author{
0.0633 \\ 0.2250 \\ 0.2400 \\ C. 2678 \\ 40.2803 \\ 0.3003
}

0.1100

0.4236

0.4601

0.4949

0.5277

0.5588

0.2597

0.6357

0.0943

0.7492

0.8025

0.3494

0.0462

0.1603

0.1752

0.1846

0.2037

0.2174

0.0434

0.1495

0.1637

0.1771

0.1901

$0.202 e$

$20.00 \quad c .15 C \quad 0.21500 . \operatorname{tet} C \quad 0.0500$

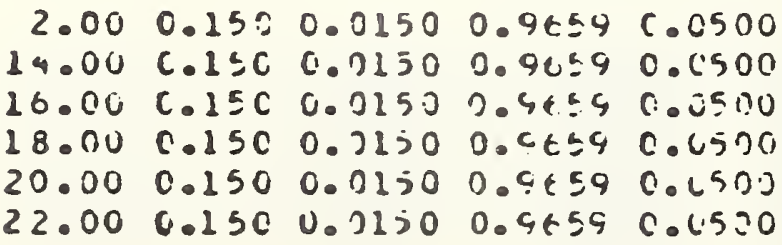

$\begin{array}{lllllll}2.00 & 0.250 & 0.2150 & 0.9962 & c .0500\end{array}$

$14.00 \quad 0.15 \mathrm{C} \quad 0.0150 \quad 0.946 .2 \quad 0.0500$

$16.000 .150 \quad 0.01500 .99620 .050$

$13.0 \mathrm{C} 0.13 \mathrm{C}$ 0.0150 0.99ez C.. (50J

$20.00 \quad 0.150 \quad c .0150 \quad 0.8942 \quad 0.6500$

$22.000 .130 \quad 0.3150 \quad 0.45 E 2 \quad 0.0500$
0.0420

0.1447

C. 1578

0.1708

0.1932

0.1 i54

0.0415

0.1432

0.1504

$0.16 \% 1$

$0.13 i j$

0.1935
0.1953

0.7922

0.8662

0.9362

1.0008

1.0636

0.2201

0.7021

0.9864

1. 0663

1.1433

1.2157

0.2350

0.9047

1.0542

1. 1441

$1 .<270$

1. 3051

0.2397

0.4839

1.0775

1.1670

$1 . \leq 527$

1.1307
0.1008

0.4021

0.4436

0.4341

0.5239

$0.5630^{\circ}$

0.1008

0.4021

0.4436

0.4641

0.5239

0.5030

0.1008

0.4021

0.4436

0.4841

0.5239

0.5630

0.1008

0.4021

0.4436

0.4541

0.5234

0.5630

0.1008

0.4021

0.4436

0.4841

0.5239

0.5330

0.1008

$0.40<1$

0.4436

0.4641

0.5239

0.5630
0.6502

32.6544

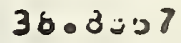

41.5134

$44.52>0$

46.9423

4.6042

39.6793

43.3195

46.6002

49.6710

52.3477

9.5702

41.3743

45.1702

40.3256

51.2240

54.7104

10.1803

$4<.2502$

40.1423

49.6742

52.9747

$53.94 \times 1$

10 . 28 y 4

42.6000

40.6325

50.2050

53.2470

50.5024

10. 3130

$42 .=073$

40.7545

su. $34=4$

$51.71 j 3$

$50.7 i>7$ 


FLOH CIA. MANIN SUPPLY CFAIN
LIS. M. CJEFF SLLPE SLLPE
ISINI ISINI

$2.00 \quad 0.150 \quad 0.0150 \quad 0.25 t t \quad 0.0250$

$14.00 \quad 0.250 \quad 0.3150 \quad 0.2584 \quad$ C.C. 550

$16.00 \quad 0.150 \quad(.01) 250 \quad 0.2560 \quad$ I. $(125,0$

$19.00 \quad 0.150 \quad 0.31500 .25 t r \quad C .0 \geq 50$

$20.00 \quad(.150 \quad 0.31>0 \quad 0.25 t 0 \quad 0.1250$

$22.00 \quad 0.1 \leqslant C \quad 0.3150 \quad 0.23 t 5 \quad C .4250$

$2.00 \quad 0.1500 .01500 .5 C C O \quad c .0250$

$14.00 \quad c .15 C \quad 0.0130 \quad 0.5 C C \cdot \quad n . C 250$

$10.00 \quad 0.150 \quad 0.2150 \quad 0.50 C 0 \quad C .1290$

$13.00 \quad 0.1 \leq C \quad 0.3120 \quad 0.5 C C .6 \quad$ C. 0250

$20.00 \quad .1 \leq 0 \quad 0.3150 \quad 0 . \pm 0 C 0 \quad .0250$

$22.00 \quad 0.1500 .32500 .5 c c 0 \quad 0.0250$

$2.00 \quad c .1500 .1150 \quad 0.7 C 7$ C r.c250

$14.00 \quad 0.150 \quad 0.3150 \quad 0.7 C 7 C \quad 0.025 J$

$16.00 \quad 0.15 C \quad 0.01500 .7 C 76 \quad 8.02<0$

$1 \% .00 \quad 1.150 \quad 0.31500 .707 C \quad c .0250$

$20.03 \quad 0.150 \quad 0.31300 .707 C \quad$ C.C25.

$22.00 \quad 0.1500 .3150 \quad 0.7070 \quad 0.0 .250$

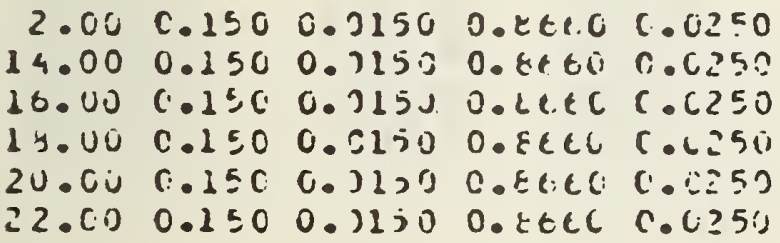

$2.00 \quad 0.150 \quad 0.02530 .9659 \quad 0.0250$

$14.00 \quad 0.15 C \quad 0.3150 \quad 0.9454 \quad 0.2 .250$

$16.00 \quad 0.1 \leq 0 \quad 0.3150 \quad 0.5 t \leq 9 \quad r .0 .250$

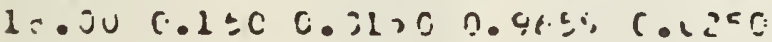

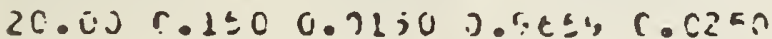

$22.060 .150 \quad 0.1120$ ?.40.5 $\quad .1250$

$2.00 \quad 0.150 \quad 0.31500 .55 t 2$ C. 0.250

$14.000 .1 \% 0 \quad 0.31$ ij 0.44 ci c.i?an

13.0 C.1SC 0.n230 0.44EZ C.1.250

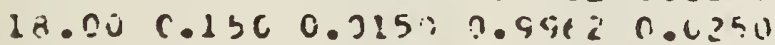

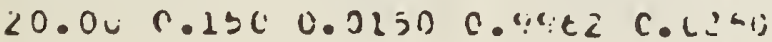

$22.00 \quad 0.1=0.01500 .094<0.4250$
DRAIN FLOH

ENTRY DEPTH

RATIO N/O.
ENTRY

ENERGY

n.
NURMAL DEPTH

H/O.
PIPE LEIGTH TI NOKAAL JEPTH. L/O.

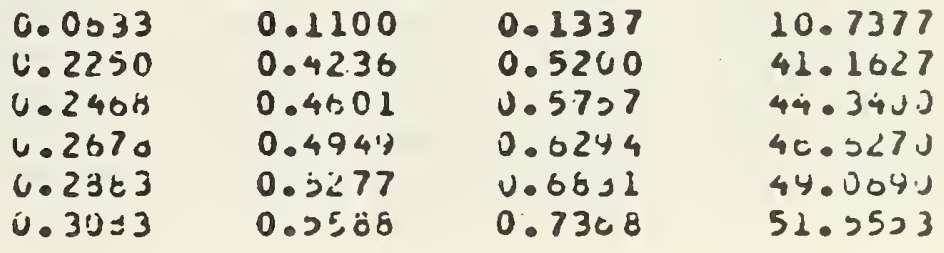

0.0515

0.1801

0.1969

0.2133

ci. 2291

c. $244 \%$

c. 0402

0.1503

i. 17,2

ن. $1=4 t$

i. 2337

c. 2174

0.0434

i. 1490

v.1637

(1. 1771

0.19נ1

0.2023

$0.04 \angle C$

C. 1447

0.1570

ن. 17 , c

(.) $1 \div 32$

L. 1124

i. 0415

c. 1432

c. 1504

U. 10y1

L. 211 3

U.1.33
0.1597

0.0357

0.0943

0.5015

0.5494

0.1953

0.7922

0.8062

0.9361

1. $003 \mathrm{H}$

1.0635

0.2201

0.9021

0.4564

2. 5663

1. 1433

$1 . \angle 157$

0.2350

0.9647

1. U532

1.1441

1.2270

1. 3051

0.2397

$0.4: 34$

1. 4775

1.1066

2. 2527

1.3307
0.7492
0.1337

0.5200

0.5757

U. 0294

$v \cdot t 031$

U. 7308

0.1337

0.52 .00

U.575?

0.6244

0.3831

ن. 7308

0.1337

0.5200

0.5727

0.0294

ग.059 1

0.7300

0.1337

0.5250

J. 5757

J. 6214

0.6531

0.7308

\subsection{7}

0.5200

0.5757

0.6244

0.6011

ग. 7 jणत 11.joul

44.7311

40.2350

56.4270

$5 j .7$ ن.jo

5 c. 4 c 1

11.9140

40.3445

5 บ・15ว

2⿺辶 $0=77$

55.7755

50.7010

12.1030

47.2074

$=1.0 \cup 13$

53.4 .515

30.9103

$54.11=7$

12.2000

$47.0<44$

$51.4 \geq 27$

$54.42 t>$

57.4212

oC. $5 \geq 67$

$12.22,1$

$4 \% .7443$

$51.50: 23$

54.2721

57.0443

OU.0521 
FLOH DIA. MAYN. SUPPLY DRAIY L/S. H. COEFF. SLCPE SLQPE (SIN) (SIN)
ORAIN FLCH EMTRY DEPTH RATIJ H/D.
ENTRY

EMERGY

M.
NORAA L DEPTH

H/D.
PIPE. LEVITH I MORMAL JEPTH. L/D.

$\begin{array}{rllll}2.00 & 0.150 & 0.0150 & 0.2588 & 0.0167 \\ 14.00 & 0.150 & 0.2150 & 0.2586 & 0.0167 \\ 16.00 & 0.150 & 0.3150 & 0.2586 & 0.0167 \\ 18.00 & 0.150 & 0.0150 & .1 .2588 & 0.0167 \\ 20.00 & 0.150 & 0.1150 & 0.2586 & 0.0167 \\ 22.00 & 0.150 & 0.1150 & 0.2588 & 0.0167\end{array}$

$2.00 \quad 0.15 C \quad 0.0150 \quad 0.50 C 0 \quad 0.0167$. $\begin{array}{lllllll}14.00 & 0.15 C & 0.0150 & 0.5000 & 0.0157\end{array}$ $16.00 \quad 0.150 \quad 0.0150 \quad 0.56 C 0 \quad 0.0157$ $18.00 \quad 0.15 C \quad 0.2150 \quad 0.5 C C C \quad C .0 .157$ 20.000 .1500 .01500 .5000 .0167 $22.00 \quad 0.1500 .01500 .5 C C C$ C.C.07

$2.00 \quad 0.150 \quad 0.0150 \quad 0.7070 \quad 0.0145$ $14.00 \cdot 0.150 \quad 0.0150 \quad 0.7 C 70 \quad c .0 .167$ $16.00 \quad 0.150 \quad 0.3150 \quad 0.7670 \quad$ C.C1.57 $18.04 \quad 0.15 C \quad 0.31500 .76 .70 \quad 0.0157$ $20.00 \quad 0.150 \quad 0.2150 \quad 0.7070 \quad 0.0 .257$ $22.00 \quad 0.150 \quad 0.71500 .7070 \quad 0.1157$

\subsection{3 \\ 0.2220 \\ 0.2468 \\ $0.267 \%$ \\ 0.2803 \\ 0.3003}

0.0515

0.1842

0.1469

0.2133

$0.22+1$

0.2449

0.1603

0.2752

0.1990

0.2037

0.2174

0.0434

0.1493

0.1637

0.1771

0.1901

0.2028

0.0420

c. 1447

0.1570

0.1700

0.1832

0.1954

0.0415

0.1432

U. 2504

0.1011

0.1323

0.1735
0.1100

0.4236

$0.4 \leq 01$

0.4947

0.5277

$0.558 \%$

0.1597

0.0357

0.0943

0.7492

0.8015

0.5494

0.1953

0.7422

0.0662

0.9361

1. 0005

1.0636

0.2201

0.9021

0.9864

1.0663

1.1433

1.2157

0.2350

0.7647

1. 0562

1.1441

1.2270

1.3051

0.2397

0.7539

1. 4775

1.1060

1.6527

1.3307

\subsection{7}

0.6000

0.6724

0.7303

0.8013

0.8647

$0.15<7$

0.5000

0.6724

0.7308

0.8013

0.2047

0.1527

0.6060

0.6724

0.7368

$2.60 \$ 3$

0.0047

0.1527

0.6030

0.6724

0.7358

0.8013

0.8647

0.1527

0.6060

0.6724

ن. 7305

0.8013

0.8547

0.1527

0.6060

0.6724

0.7308

$0 . \varepsilon 013$

0.0347
11.0444

30.0321

40.1312

41.6323

42.87 .14

43.7873

11.8447

41.6012

44.0872

42.4453

$47.5 \div<5$

40.7473

12.2030

43.2469

40.9004

47.9257

44.6731

51.024 .1

12.3939

44.4383

40.5291

49,3057

50.2755

$5 \mathrm{~L} .2072$

12.4929

45.2075

40.4107

$50.15 \div 3$

$50.57 i 1$

$5<.5023$

14.5213

45.5317

4c. ijडt

5U.37J 3

5U.0:? ?

2द. $4=07$ 
FLOH DIA. MANN. SUPPLY TFALN LIS. H. COEFF SLTPE SLLPF (SIN) (SIN)
DRAIN FLOH

TNTRY JEPTH

PATIO $\triangle$ IC.
ENTRY

ENERGY

M.
NORMAL DEPTH

H/D.
PIPL LEINUTH TC NOP.ALL DEPTH.

L/O.
$2.00 \quad 0.250 \quad 0.0250 \quad 0.25880 .(125$

$14.00 \quad 0.150 \quad 0.31500 . Z 58 t \quad C . C 125$

$16.000 .1 \leq C \quad 0.0150$..

$1 E .0 J \quad 0.150 \quad 0.0150$ D. Z5EC 0.012 2

$20.00 \quad 0.250 \quad 0.7150 \quad 0.2580 \quad C .0125$

$22.00 \quad 0.1500 .31500 .2$ EEe (i.Ul?

\subsection{3 \\ 0.2250 \\ U. 2400 \\ U. 2078 \\ ט. 2303 \\ 4.3053}

$0.021 \%$

C. 1201

0.1969

0.2133

c. 2271

U.2447

v. 0402

0.1603

$0.17>2$

C. 1896

U. 2337

v. 2174

0.0434

U. $14+6$

ن. 1037

0.1771

ט.1.1 1

$0.20<0$

0.0420

0.1447

U. 1070

ט.17U⿺

0.1332

ن. 1 th 4

$\cup .0415$

ن. $143 i$

‥1504

$0.11) 11$

6. $1: 13$

0.175
0.1100

0.4233

0.4001

0.4944

0.5277

0.3509

0.1597

0.0357

0.6943

0.7442

0. त्य 25

0.5494

0.1953

0.7022

$0 .=662$

0.9361

1.0003

1.0630

0.2201

0.7021

0.4564

1. vंगु

1.1433

$1 .<157$

0.2350

0.4047

1. $u=82$

1. 1441

1. 2270

1. 3052

0.0397

0.2033

1. 0775

1.16h

1. 2327

1.3307
0.1601

0.6702

0.7534

0.8207

0.8999

0.9722

0.1631

0.6732

0.7534

U. 2207

0.8999

0.9722

0.1601

$0.67=2$

0.7514

0.9207

0.6994

0.9722

0.1601

0.67 .2

0.7534

U. 6207

$3.89+9$

$0.97<2$

$0.16=1$

$0.67,2$

0.7534

J. 8207

נ.

0.472 ?

0.1031

$0.67,2$

0.7594

J.020 7

U. 34,4

$3.97<2$
1C. 3002

29.3 $3>1$

$24 \cdot 123$

24.77:7

(4. $42 j J$

24.0737

11.1253

32.2441

34.53 .6

$37.417 j$

$31 \cdot 4=74$

34.10007

$11.40 ? J$

33.3537

30. टगU1

37.3324

$31.5<J 3$

32.5115

11.6752

$33.337 j$

$37 . i 3 \geq 0$

3) 7:140

24.7345

36.4130

$1+\ldots 3+1$

34. I2ن1

$37 \cdot 5 \therefore, 0$

3 ㄷ.. $3=?$

27.

$30 . .204$

11. Y.,

34. -

37.6 .27

$3=.1:-2$

cC. isid

$30.4<34$ 
FLOH RIIA. MAYN. SUPPLY DEAIY L/S. H. COEFF SLCPE SLOPE (SIN) (SIN)
DRAIN FLOSH ENTRY

ENTIR OEPTH ENERGY

RAT $10 \mathrm{M} / \mathrm{C}$.
H.
MORAAL DEPTH

H/D.
PIPE LEVGTH NORMAL UEPTH L/O. $\begin{array}{lllll}2.00 & 0.250 & 0.0130 & 0.2568 & 0.0500 \\ 14.00 & 0.150 & 0.1110 & 0.2580 & C .0 .500 \\ 16.00 & 0.15 C & 0.0130 & 0.2586 & 0.0500 \\ 18.00 & 0.15 C & 0.0130 & 0.2588 & 0.05 n 0 \\ 20.00 & 0.150 & 0.0130 & 0.2586 & 0.0500 \\ 22.00 & 0.150 & 0.0150 & 0.2580 & 0.0500\end{array}$

$2.00 \quad 0.150 \quad 0.2130 \quad 0.3 C C 0 \quad 0.0500$

$14.00 \quad 0.150 \quad 0.131300 .500 . \quad C .0500$

$16.000 .15 \mathrm{C} 0.0130 .50 \mathrm{CC} C .0500$

$18.00 \quad C .150 \quad 0.7130 \quad 0.50 C 0 \quad c .050 n$

$20.00 \quad 0.15 C \quad 0.31300 .5 C C=0.1 .500$

$22.00 \quad 0.250 \quad 0.21300 .5000 \quad c .(5000$
$2.00 \quad 0.150 \quad 0.31300 .8 \in t C \quad 0.0500$

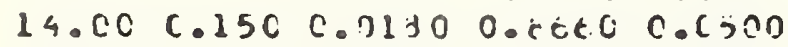

$16.00 \quad 0.15 \mathrm{C} \quad 0.31300 . \operatorname{tetc} 0.0500$

$18.00 \quad 0.15 C \quad 0.31300 .8 t t \bar{C}$ C.C500

$20.000 .130 \quad 0.31300 .1 t t C \quad 0.050 C$

$22.00 \quad 0.1500 .11100 . \varepsilon e t C \quad 0.05100$

0.0710
0.2551
0.2600
0.3040
0.3274
0.3508

0.0577

0.2035

0.2220

0.2426

0.2600

0.2701

0.0512

0.1810

0.1 is 1

0.2145

0.2306

0.2463

\subsection{6}

0.0906

0.3414

0.3707

0.3957

0.4234

0.4485

0.1296

0.5071

0.3527

0.5952

0.6347

0.6714

0.1579

0.0293

0.6863

0.7413

0.7420

0.5400

0.2777

0.1691

0.1349

0.2013

c. 2152

0.2276

0.0470

0.1632

0.1763

0.1730

0. 2074

0.2213

0.0465

0.1615

0.1764

0.1920

$0.20 \$ 2$

0.2109

0.9537

0.1596

0.7653

0.0371

0.71547

0.4674

1.0291

0.1929

0.7805

0.3548

$0.4 \overline{2} 24$

0.4872

1.0500
0.1201

0.4547

0.5003

0.5552

0.602 :

0.6479

0.1201

0.4547

0.5083

0.5552

0.6021

0.6479

0.1201

0.4577

0.5083

0.5552

0.6021

0.6479

0.1201

0.4577

0.5003

0.5552

0.6021

0.6479
0.2201

0.4547

0.5053

0.5532

0.6021

0.6479

0.2201

0.4547

0.5003

0.5502

$0.60<1$

0.6479
:

0.4405

25.7215

20.0527

29.7732

31.7143

33.3409

7.2413

20.0015

31.2745

33.2354

35.4241

37.20 .13

7.4375

24.9402

$3<.07>?$

$34.77<1$

37.07 .3

39.0250

7.5925

30.0337

$33.42: 3$

35.53 .57

$37.74 ; 3$

34.9742

7.0724

30.96 .30

33 .

30.0. 2

30.3134

46.4450

7.0424 31.17074 33.9607

$30 \cdot 1: 23$

$30 \cdot 3+i 7$

40.3545 


\section{FLOH TIA. MAYN. SUPPLY OPAIN H. CUEFF SLCPE SLLPE (SIM) (SIN)}

DRAIN FLEH ENTRY ENTRY DEPTH EINERGY RATIO H/L. n.
NORMAL DEPTH

H/D.
PIPE LEYGTH TO NOKMAL UEPTH. L/D.

\begin{tabular}{|c|c|c|c|c|}
\hline & $\begin{array}{l}0 \\
0 \\
0 \\
0\end{array}$ & $\begin{array}{l}130 \\
139 \\
140 \\
130\end{array}$ & $\begin{array}{l}0.2 \\
0.2 \\
0.2 \\
0.2 \\
0.2\end{array}$ & \\
\hline
\end{tabular}

$2.000 .15 C \quad 0.01300 .50 C C$ C. C 250

$14.00 \quad C .150 \quad 0.3150 \quad 0.3 C C C \quad C . C 250$

$16.00 \quad 0.15 C \quad 0.31500 .50 C 6 \quad(.6250$

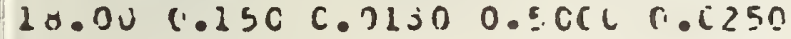

$20.00 \quad 0.130 \quad 0.01300 .5 C C L C .0 .250$

$22.00 \quad 0.150 \quad 0.0130 \quad 0.3 C C C \quad C .1 .250$

$2.00 \quad 0.250 \quad 0.3130 \quad 0.70 .7 C \quad 0.0250$

$14.00 \quad 0.156 \quad 0.31300 .7(7 C \quad C .075,0$

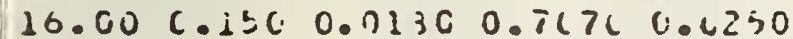

$18.02 \quad 0.1300 .01300 .7070 \quad 0.0[251]$

$20.00 \quad 0.150 \quad 0.11300 .7676 \quad c .0250$

$22.00 \quad 0.150 \quad 0.0130 \quad 0.7070 \quad c .4250$

$2.000 .150 \quad 0.01300 .8 . \in C C$ C.01250

14.00 C.1SC 0.2132 C.ttt: C.C25n

$16.00 \quad C .1 \leq C \quad 0.31 \neq 0$ n.tetc C.C250

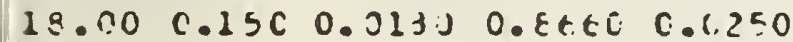

$20.00 \quad 0.15 C$ G. $013 . J 0.8 t . t e r .1 .250$

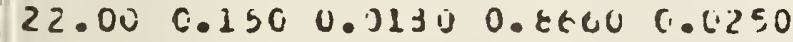

$2.000 .15 C \quad 0.0150 \quad 0.9654 \quad 0.0250$

$14.00 \quad 0.1300 .3190 \quad 0.5454 \quad C .0250$

$16.00 \quad 0.1500 .0130 \quad 0.90 \leq 9(.0250$

$1 \approx .00 \quad 0.15 C \quad 0.31 y 0 \quad 0.4 c \leqslant c_{i} \quad 0.0 .250$

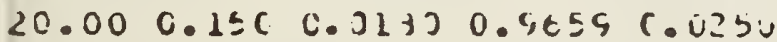

$22.00 \quad .15 C \quad 0.12300 .5459$ C.1253

$2.00 \quad C .130 \quad C .21300 .494=\quad(.0250$

$14.00 \quad 0.1500 .11310 .996 \quad(.0250$

16.00 C.136 0.171300 .9962 C. 2250

$10.00 \quad 0.150 \quad 0.21300 .946 \bar{C} \quad .024 .1$

20.0 r (.15C C. $311130.9042 \quad .0250$

22.00

$\begin{array}{ll}0.0710 & 0.0906 \\ 0.2551 & 0.3414 \\ 0.2500 & 0.3707 \\ 0.3040 & 0.398 .7 \\ 0.3279 & 0.4235 \\ 6.3508 & 0.4485\end{array}$

0.0577

0.2035

0.2220

0.2410

ن. 2000

$\dot{0} .2701$

0.0510

0.1810

0.1911

0.2145

c. 2306

0.2403

0.0486

0.1691

0.1344

$0.20 \cup 3$

6. 2122

i. 2290

0.0470

0.1632

0.1703

U. 1950

0.2074

J. 2213

C. 0403

0.1615

0.1704

0.1910

0.25\%2

0.2104
0.1296

0.3071

0.2527

0.3552

0.0347

0.6714

0.1379

0.0293

0.0263

0.7413

0.7920

0.0400

0.1777

0.7157

$0.7 \pm 13$

0.0430

$0 . y 015$

$0.75 \& 7$

0.1896

0.76 .53

0.0371

0.9047

0.4674

1. 2281

0.1729

$0.7=08$

$0.0=48$

$0.12 \geq 4$

0.1072

$1.0 \leq 00$
0.1508

0.5972

0.6616

0.7201

$0.7 \mathrm{dJ6}$

0.3511

บ. $15 \cup 8$

0.5972

$0 . t .616$

0.7201

v. 7500

0.0511

0.1508

0.5972

0.6016

0.7201

0.7306

0.8511

$0.15 \cup 8$

0.5972

0.0616

0.7201

0.7506

0.0511

0.1508

0.5472

0.0610

0.7201

$0.7 \pm 06$

4.0511

0.1503

0.5472

0.6016

0.7201

j.781io

0.8512
7. 3353

25.3315

20.6207

27.5 गुय 4

26.5429

$29.134 . J$

7. 4485

26.0321

24.5423

31.0134

32.0100

32.2270

8.2235

24.24 .15

$30.97 \leq 1$

$3<.5272$

$33.04,7$

34.6172

$0.37=4$

24.9754

31.7315

$33.34,7$

34.5318

$32.37 \div 9$

6.4503

30.3143

32.1245

33.7507

$34.9=55$

30.نว ?

0.4773

36.4130

$3<.2 i>0$

33. 30u

33.1131

30.2'si 
FLOH CIA. MAYN. SUPPLY TPAIN L/S. H. COEFF SLQPE SLCPE (SIM) (SIN)
ORAIN FLOH ENTRY ENTRY LEPTH ENERGY RATIO HID. . M.
NORMAL DEPTH

H/O.
PIPE LEAETH I NORMIL UEPTH。 $L / D$. $\begin{array}{llllll}2.00 & 0.150 & 0.0130 & 0.2588 & 0.0167\end{array}$ $14.00 \quad 0.1500 .0130 \quad 0.25860 .(13)$ $16.00 \quad 0.1300 .01300 .256<0.0167$ $19.00 \quad 0.150 \quad 0.01300 .258 t \quad 0.0167$ $20.00 \quad 0.15 \mathrm{C} \quad \mathrm{C.0} 31100.256 \mathrm{C} \quad .0167$ $22.00 \quad 0.150 \quad 0.0130 \quad 0.25680 .0137$

$2.00 \quad 0.150 \quad 0.0130 \quad 0.50000 .0167$ $14.00 \quad(.150 \quad 0.31300 .5 C C O \quad .0167$ $16.00 \quad 0.150 \quad 0.9130 \quad 0.5 C C C \quad 0.0167$ $18.000 .1500 .11100 .5000 \quad 0.0167$ $20.00 \quad 0.150 \quad 0.3180 \quad 0.5 C 0 C \quad 0.0167$ $22.00 \quad 0.150 .21300 .50 C C \quad .(267$

$2.000 .150 \quad 0.01300 .76740 .0167$ $14.00 \quad 0.150 \quad 0.21300 .7070 \quad 0.0137$ $16.00 \quad 8.150 \quad 0.01300 .7 C 70 \quad c .0167$ $1.000 .1300 .31300 .7070 \quad 0.0167$ $20.00 \quad 0.150 \quad 0.1150 \quad 0.7070 \quad 0.0167$ $22.00 \quad 0.1500 .3190 \quad 0.7 C 7 C \quad 0.0167$

$2.00 \quad 0.15 C \quad 0.01300 .8 t \in C \quad 0.0167$ $14.00 \quad 0.15 C \quad 0.2130 \quad 0.8 t .6 C \quad 0.0167$ $10.00 \quad 0.15 C \quad 0.0130 \quad 0 . t 6 t C \quad 0.0167$ $15.00 \quad 0.1500 .113 .30 . \varepsilon e 600.0167$ $20.00 \quad 0.15 C \quad 0.01300 . t 6 t 0 \quad 0.4167$ $22.00 \quad 0.150 \quad 0.31: 00.8 t e c \quad C .0367$

$2.00 \quad 0.150 \quad 0.21300 .94590 .0167$ $14.00 \quad 0.15 \mathrm{C} \quad 0.01300 .9659 \quad 0.0167$ $16.00 \quad 0.150 \quad 0.01300 .9454 \quad 0.1267$ $18.00 \quad 0.1 \leqslant c \quad 0.31 .31) \quad 0.96 \leqslant 90 .(167$ 20.000 .1300 .0130 0.9t 590.0167 22.00 C.15C C.21,u 0.96 .59 r.C.C167

$2.00 \quad 0.150 \quad 0.01300 .9962 \quad 0.0167$ $14.00 \quad 0.15 C \quad 0.3130 \quad 0.7962 \quad 0.0187$ $10.00 \quad 0.150 \quad$ C. 01500.99420 .0167 $18.00 \mathrm{C.1} 500.31340 .99420 .4137$ $20.0 .0 .15 C \quad 0.01300 .494 z \quad 0.0137$ $22.00 \quad 0.1 \leq C \quad 0.01300 .99 C 2 \quad 0.0167$

\subsection{0 \\ 0.2551 \\ 0.2030 \\ 0.3040 \\ 0.3279 \\ 0.3508}

0.0577

0.2035

0.2228

0.2416

0.2600

0.2701

0.0518

$0.1+10$

0.1901

0.2145

0.2306

0.2463

0.0406

0.1691

0.1549

(:. 2003

U. 2152

0.2246

0.0470

0.1632

c. $17 \pm 3$

0.1930

v. 2074

U. 2213
0.0906

0.3414

0.3707

0.3987

0.4238

0.4485

0.1296

0.5071

0.5527

0.5952

0.0347

0.0719

0.1574

0.6293

0.0863

0.7413

0.7920

0.8400

0.1777

0.7157

0.7613

0.0430

0.4018

0.4527

0.1896

0.7653

0.0371

0.9047

0.4674

1.0281

0.1929

0.7808

0.8544

0.4224

0.9872

1. 4500
0.1725

0.6978

0.7759

0.8521

0.9272

1. 0000

0.1725

0.6978

0.7759

0.3521

0.9272

1.0000

0.1725

0.6978

0.7754

0.8521

0.9272

1. 0000

0.1725

0.6978

0.7759

0.5521

0.9272

1.0000

0.1725

0.6973

ن. 7759

0.3521

0.9272

1.0000

0.1725

0.6978

0.7759

0.3521

0.9272

1.0010
6.5632

15.1757

16.7734

$16.10+7$

24. 2745

15. $33=7$

$7 \cdot 1756$

22.3477

18.7341

10.8500

$10 . \$ 367$

17.4717

7. 4063

21.7122

$19.31 \div 7$

20.8320

25.0935

17.9951

7.6178

21.557 .7

19.3045

21.5153

22.3173

17.5447

7.6935

22. 2225

19.2514

22.1145

$22.27 \mathrm{~J} 7$

17.5154

7.71 .74

22.1700

14. 28.22

2L.:4\%

$20^{\prime} .2101$

17. 蚂了? 
APPENDIX 2

DESCRIPTION OF PROGRAM PROFIL 2 
Program PROFIL 2

This appendix presents a complete print out of this program together with sample input data. The program was run on the NBS CBT Perkin Elmer 732 computer.

The program accepts data in SI units with the exception of flow rate which is read in liters/second and corrected to $\mathrm{m}^{3} / \mathrm{s}$ within the program。

The program is effectively the first section of the hydraulic jump prediction program fully described in reference [1]. For this reason no detailed flow chart is included in this report. 


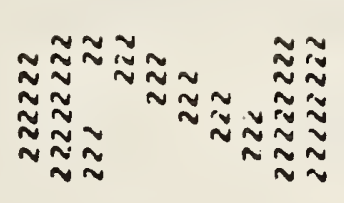

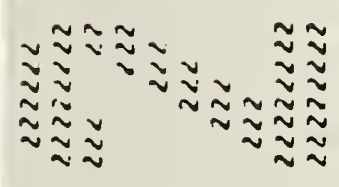

J

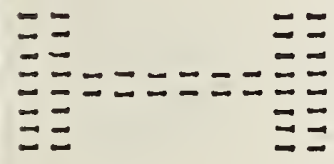

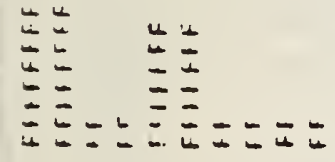

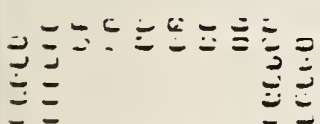

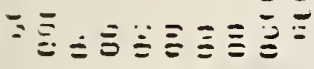

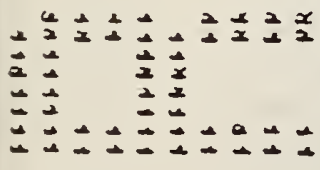

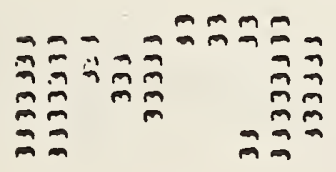

952

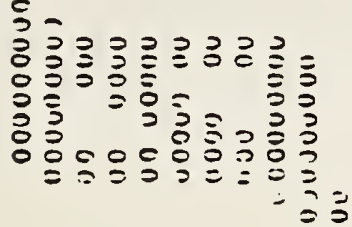

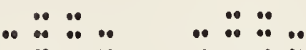

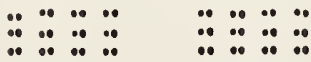

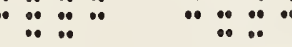

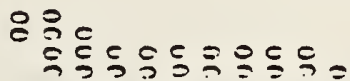

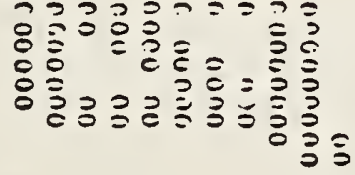

ํํㄱ

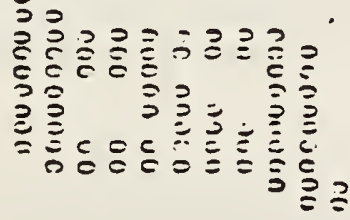

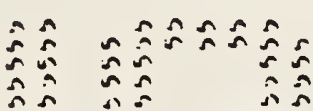

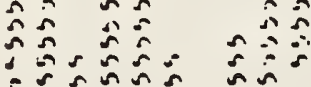

:

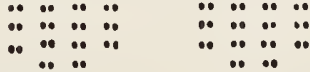$$
\bullet \bullet
$$
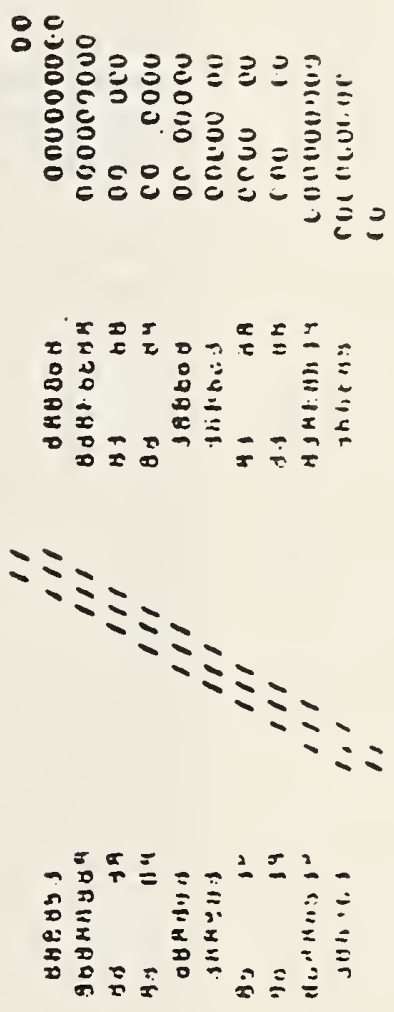

ㅇำ

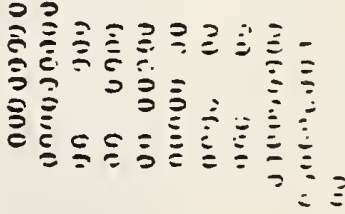

ミミミン<smiles>C=C=C=C=C=C=C</smiles>

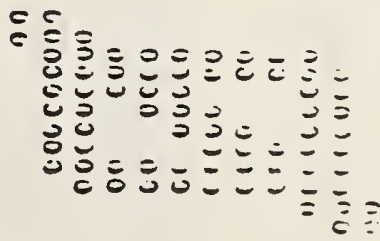

こニニニニニニニきき 
PROGRAM PRCFILE CALGJLATES THE hateá SUKFACE PRLF ILE IN SUPERCRITICAL FLIA FRIJA AN FNIRT EONLITIDN REPRE SENIINC TERAITAL CIINCITITINS IN A STEE SUPPLY PIPE

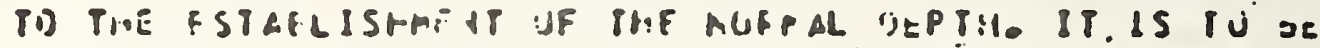
NDTED THAT ThIS TZAMjITIUN CUES NOBT SRZZUIKE A HYERAULIC JUEP AS THE PLLH IS JUPEXCFITIC.AL AT ITS FULLY DEVELOPED CEPTH.

.)(MENS ION $\$ 12,10 C), F(2,100), 1$ ( 14$)$

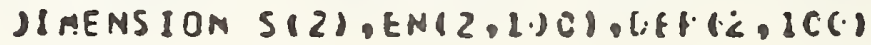

I VTECER SHAPE

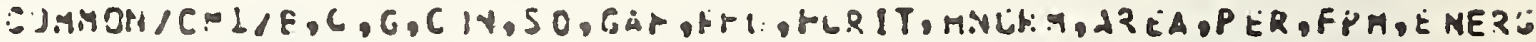
GIMMON/CRE/ SHAPE

SUMMON/CHZ/IZ

PEAT(4,70LISTAPE

702 F.र.MAT(1)

REAO(4, IZU) MA,NS,ILON

REAJ(4, 121$) F, L P, C . E V$

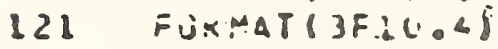

$\dot{j=9}$. 41

I $R U N=0$

$i \angle=0$

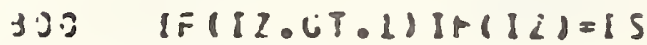

$I L=I i+l$

IFIII. $6 T .2) 12=1$

READ $14,10 \because 2 P L, S C, C, H C I N T, E N Z$

$5(I 2)=50$

6. IF ICEN=1 THE FLCH IVILYSIS IS FLSI? JV AA INPUT EVERUY AT

- E.TTRY TIT THE PILD IL IOE PIPF, TEFP. RNE. S. TILARLY THE LUSE

C BJEFFICIENT LI PIP ENTZY MAY LF EYPSESSEU AS A FACTJKOUEN.

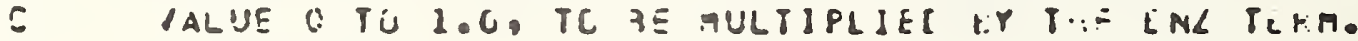

C IS THE ENTFY FLCW SEPTH IS TC \&F CALCULATES FKIA ENLEEN

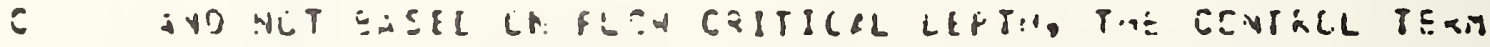

C TCUNT IS SET TO 1.J. THE WIHEK PAMAMETERS REFEK TL PIPE ELJH

C RATE AND UIFERSIOND.

loJ = उरMATISF,C.4)

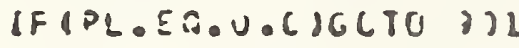

C PLOPIPE LENCTH BOAI:TH, BPAFCNMIPI; CUEFF, SDOSLUPE,

C J-FLOHZATE IN LIS TJ JE USEC IN F\$3/S, MEUNT-CCNTROL LFPTH

C SET TU LEKL IF CFITICAL JEPTH ASSLMED.

$j=2 / 160.90 \%$

$\langle H J=10$ ONO.

$\therefore . J N=0 . P \$ 2 / S C$

SA.T = C QRHC

C PQUGEAA CUMPTPLL DATA.

153 F JRAAT(3)

C VY-SILE IJF THE UH STEP IN SIPFSCNS DULE ONS-NU.

- JTEPS IN LEFTH CALCOICON LETLFPINES AHETMER ENEKLY

C LIPUT OR UPSTKEAM ILIPE IS USEC, CFN IS THE ENCKGY

C IJSS FACTUK FUR THE PIPE EATKY, IN EI T.IER ENTRY CASE.

C ENZ-INOUT ENEFGY 10 QEPLACE SLIPE IF ICOY $=1$.

IF (ICDN.tQ.L) COTO GIJ

3UTO 652

$650 \quad \Xi N L=D E N \triangle E N 2$

¿ALL GOUNOIENL, $, B, B, H I$

$L L=2$

301018

651 EUPTINUE

IFIHCIINT.EO.1.0) COIT 17

j.נT: 1 . 


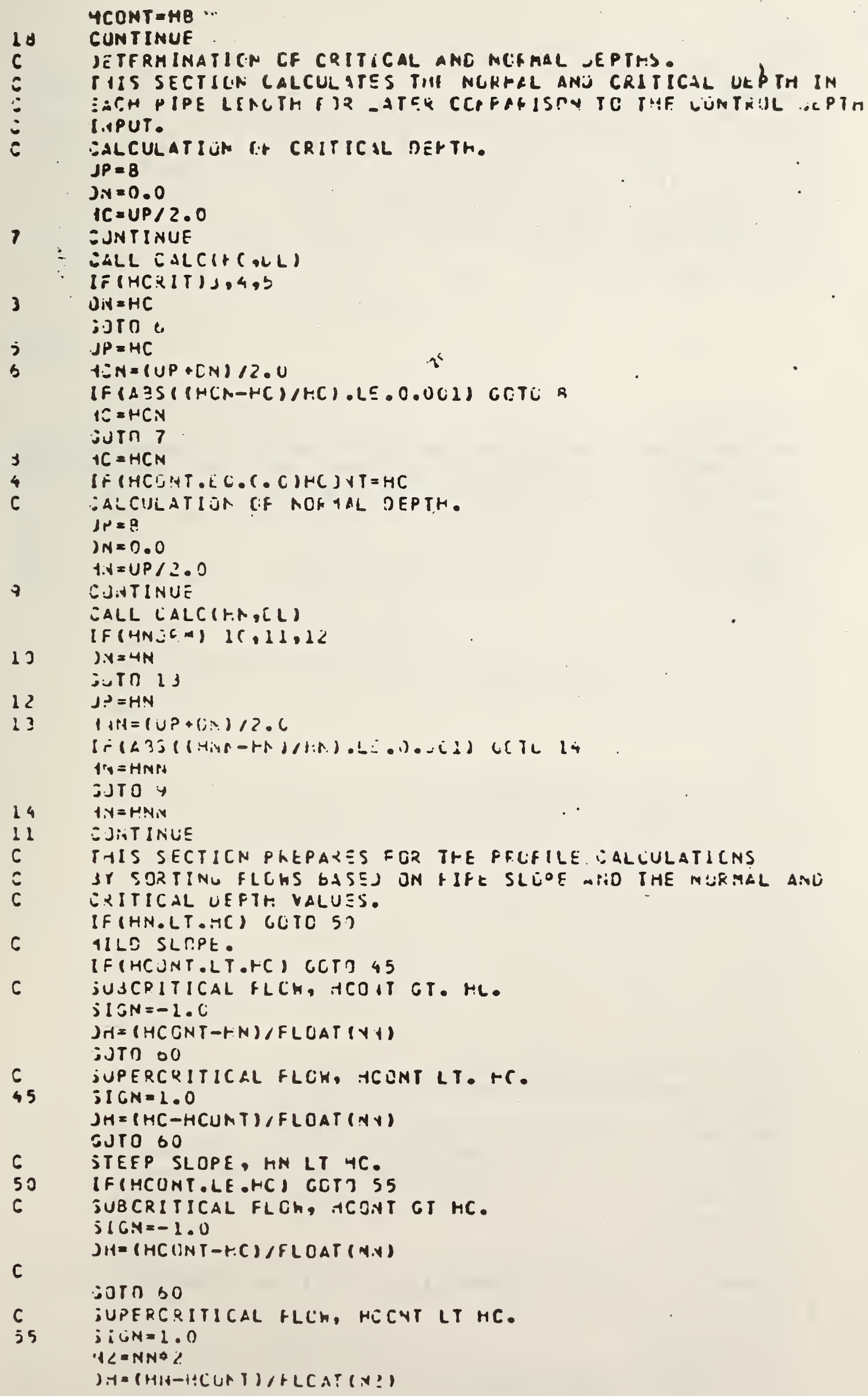


$1 S=1$

IF (II.EO.1) CCTC 347

I MCONT

:ALL CALCIR, ML

$\therefore=$ ENED?

$F .1=F P M$

(F (IL.CT.L)F(IL-1, I S) =F

IF(II.ED.21X(1, IS) =).)

[F(I].GT.1)CEP(

iF $(1) \cdot C T \cdot 1) E N(1 i-1,15)=i$

IF(!2.FL.Z) (LTC 11 ?

5
6
6
6
6
7
6
6

\section{CUNTINITE}

6

IHE APP

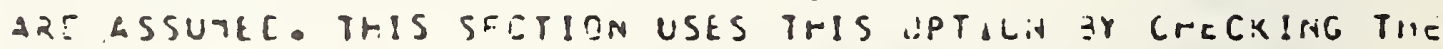
PIPF NUAEEF,IL,ANJ THE VALIIE IF ICIN AUICH IS SET TD C. $H=H A$

ZALL CELCIH,IL)

ENCJ =ENÉR.

TENT = TR

EENT $=E N G C$

octio a do

$\hat{6}$

c

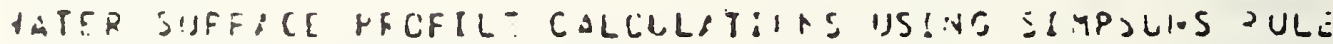

TJ FYALUATE ITE IMTOZPIL.

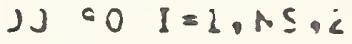

$\Sigma L O=S L$

is $=15+1$

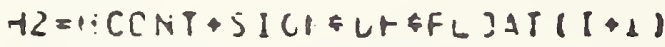

$13=H C J N T+3 I C H \& F L C A T(1)+54$

CALL CALC(r., IL)

iALL $I A L C(1 E \bar{C}, L L Z)$

$\therefore A L L C M L C(r Z, L L Z)$

$J A=D H \leqslant(? L+(L Z+4, C+1 L 3) / 3, C$

$S L=S L+: X$

$1=\mathrm{H2}$

SALL CALCIH.OCL)

$\equiv=\sum N E R C$

$F . Y=F P M$

$x(1, I 5)=5 L$

$J E P(1,1 S)=r$

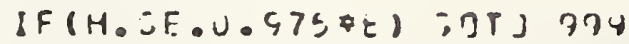

J? LONTINUE

马Q9 CONTINUE

$X F I N=X(1, I 5)$

HFIN $=H . Y$

$j=0 \$ 1000 . C$

HENT $=$ MENT/E

HF IN = HF IN/B

IF (HF I.Y.CT.C.449) HFI IN $=1 . C$

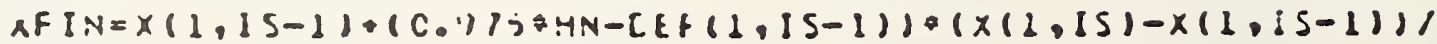

1 (DEP(1, IS)-[EP(1, IS-1)

$\angle F I N=X F I X / P$

ISIT $=1 E U . Y+1$

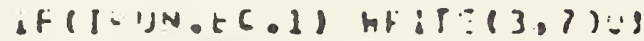

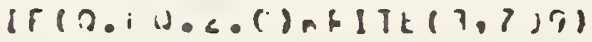


HRITE $\left(3,70110,8, R H_{0} S 11\right), 5121$, HENT, EENT, HF IN.XFIH

701

700

FRRMATI 1CX,F6.2,F6, 3, 3F 7.4, 3F 10,4,F12. 4)

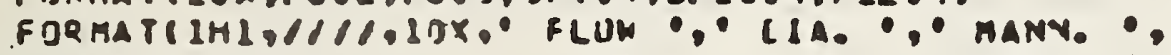

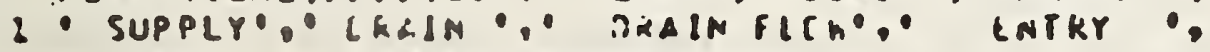
2. HORMAL $"$ PIPE LENGTH TL.,

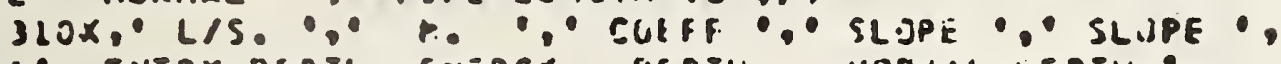
$4^{\circ}$. ENTRY DEPTH ENE?TR DEPTH MISPAAL LEPTH.",

\section{$1.29 \times 0^{\circ}$ (SIN) (SIN) .}

3. PATIO M/C. H. H:/C.

IFIJKUN.EC. 3t.) IKUNA.J

C.JT ?. อ.

QCL CUNTINUE

\section{ENO}

SUBRDUTINE RELNCIE, 2, 3, HR)

LIVTEGER SMGPE

CJAMUN/CMZ/STAPE

5
6
6
$\therefore$

SUSRTUTINE ECI NC CALCJLATES THE ENTRY CDIALITIUN TU THE IILD SLTPE FIPE IIL=ZI aY KEFLEEPLE TJ THÉ ENEXCY AT JISCHAKGE FFIM THE STFE'P SLCPE PIPT IIZ=1), UR SIMPLY EROM THE SNEPEY INPIIT UATA IF THAT MOJUE IS CHUOSEN IY THE LiALT LF ICIY = I IN THE INITIAL ÏEAD STATEMENTS. PIPE CRCSS SICTICN IS CENTHCLLET EY TIS VALUE i.F

TERM SHAPE, I=hECTAVOILAR CK $\angle=$ CIRCULAK, IN THL IAPUT. $j=9.82$

IFISHAPE.OT. 11 G[T.] 2

$Y 1=0.0$

$H \bar{O}=C /(3 *(2, C+()[1+2 . j)$

). $t=H_{1:} / 2 ? \cdot 1 \cdot \mathrm{C}$

$-1 x=4.4 \% / 2,0$

) $75 \quad I=1,1 \mathrm{CC}$

IFI).CR. I) $Y]=4$

$1 x=1 \cdot x+\int_{14}$

$P=(0+z) /(z-(+C)+(1+\neq+\alpha) \neq z) \neq(H X-E)$

IFII.ED.LICTTC 75

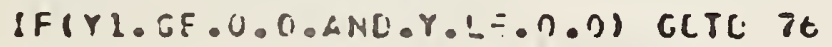

IF(YL.LE.U.C.ANCEY.IE.J.U) CCTC TE

$7 j$ EJMTIITUE

$76 \quad$ is $=4 x$

I.) 101

5 I.JNTINLE

I×J.0

$\equiv C l=0.0$

JELH=E $/ 200.0$

$2 I=3.142$

$s=8 / 2.0$

$3 \quad T=H+C E L H$

IF (H.LT.R.) THE TA=2. OFATANISCKT $(1: E(?-H)) /(K-M))$

IF (H.EO.R) THETA=PI

IF (H.CT.R) THETA=PI 2. C*ATANI( $(H-F) /(S 2 R T(H *(B-H)))$ )

IREA $=(18 * 2) / 6 . C)+(T H E T A-S I N(T H E T A) ;$

$\Xi C 2=H+(O * 2) /(1 A F E A * 2)+2.0 * C)$

IF(ECL.LE.E.AND.ECL.IE.E) CCTC a

$E C L=E C 2$

उUTा 3

$4 \quad 4 d=H$

1 IUNTINUE

RETURN

SND

JUBPRUTIIL CALCIH,I)LI

C. IUSROUTINE CALC IS USED THETLCRILT TH. PIUCRAM TG

JETEOMINE THE HLI,H-DIPL PAFARLIHLS SUGH AS FLLIN 
6 IN THE EISECTICN HETHJO CALCLLATILN OF NORHAL ANO

CRITICAL DEPTHS IN FLCH OF THE PIPE LEMGTUS. -.

IN THE CIRCULAR PIPE CRCSS SECTILIN CASF IT ALSO

GALCULATES SLGTENDFD ANGLE AMU TME MATER SURFACE

IIITH AS UEFIF ChANJËS.

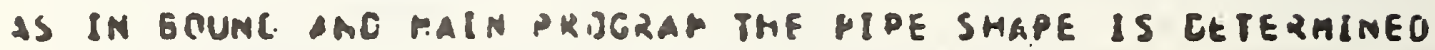

iY THF VALLF CF TH: iSOM SHAFE IMPIST AS LATA.

INTERER SHAPE

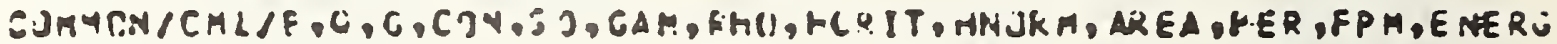

OUM IDN/CA $2 / S R \angle P E$

BMHDN/TMS/I?

IFISHAPE.CT.1)CLTO 1

IF I I Z,EO.J.ARE, H,CE. 3$) H=B$

IRE $A=H * 8$

$P E R=\underline{P}+2 \cdot 0 * 1$

IC $2 / T=1.0-(C 5 \div:) \div B /(15+A 25 A-3)$

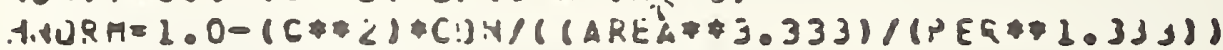

$\left.J_{L}=H C R I T / P H N C F M+5 O\right)$

$F P M=(S A-A K E \& H / 2 . D)+(X H T C+C / A F(A)$

$E N E R C=H+(U * Z) /((A R \leq 4 * 2) * 2 \cdot C * C)$

SUTा 2

1

<2 $\Rightarrow+C .5$

$P I=3.142$

IF (8Z,EO.3.APL.R.GE. I) rOTL ZC

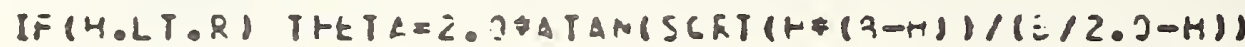

IF $\left(H_{0}[G, K)\right.$ THE TA=PI

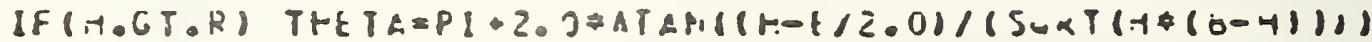

-งTO 22

$20 \quad y=j$

THET $A=2 \cdot 0 F F 1$

AREA $=P\{\neq(\varepsilon / 2 \cdot v) \leqslant 2$

$P E R=P I \div 9$

<.j $=k / 2.0$

juts

?? CONTINUE

AREAE( $(B A \bar{C}) / E . O)$ (THETA-SIN(THETA))

PER $=9+T H E T A / \bar{L} \cdot U$

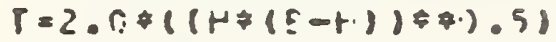

ALRIT $=1.3-(C+2.0) \leqslant T /(G+\Delta R E A+3)$

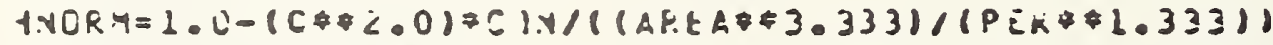

)$L=H C K I T /($ HINCKA SOSO)

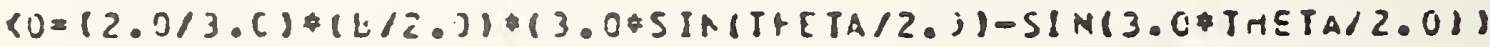

1/ $4.0=(T H=T A / \angle 0 C-0.5 \neq S$ IN(THETA) )

21 to $A R=\times O+M-t / Z \circ 0$

$F P M=C A .7 \$ A R E A F R E A R+R H C+U \div C / A R E L$

$\Xi V E F C=H+(G * 2) /((A 2 E A * 2) * 2 \cdot C * C)$

2

SUTTINUE

SETURA

ENO 
Sample data program PROFIL 2.

Line 1. SHAPE, Format I3.

Geometry indicator 2 = circular pipe

$\nabla \nabla 2$

Line 2. NN, NS, ICON, Format 314

NN - dh interval on depth profile

NS $-\mathrm{N}^{\circ}$ calculation steps, max., Simposn Rule

ICON - 2 assumes terminal conditions in approach pipe $\nabla \nabla 30 \nabla 200 \nabla \nabla \nabla 2$

Line 3. B, RM, DEN, Format 3F10.4

B - pipe diameter, RM - Manning Coeff.,

DEN - entry energy loss coefficient

$\nabla \nabla \nabla \nabla 0.1500 \nabla \nabla \nabla \nabla 0.0090 \nabla \nabla \nabla \nabla 1.0000$

Line 4. PL, SO, Q, HCONT, ECZ, Format 5F10.4

PL - pipe length, SO - pipe slope, Q - flow rate

HCONT - control depth indicator, 0 value

indicates upstream critical control,

ENZ - entry energy, zero unless ICON $=1$.

$\nabla \nabla \nabla \nabla 40.0000 \nabla \nabla \nabla \nabla 0.0070 \nabla \nabla \nabla \nabla 4.0000 \nabla \nabla \nabla \nabla 0.0000 \nabla \nabla \nabla \nabla 0.0000$

Note, 1 ine 4 is approach pipe data.

Line 5. PL, SO, Q, HCONT, ENZ, Format 5F 10.4

HCONT $=1$ indicates entry depth as control

Line 5 is test pipe data

$\nabla \nabla \nabla \nabla 40.0000 \nabla \nabla \nabla \nabla 0.0250 \nabla \nabla \nabla \nabla 4.0000 \nabla \nabla \nabla \nabla 1.0000 \nabla \nabla \nabla \nabla 0.0000$

Line 6,$7 ; 8,9$; etc repeat format 4, 5; for all test cases

Last line. PL, SQ, Q, HCONT, ENZ, Format 5F10.4 zero values terminate run.

$\nabla \nabla \nabla \nabla 0.0000 \nabla \nabla \nabla \nabla 0.0000 \nabla \nabla \nabla \nabla 0.0000 \nabla \nabla \nabla \nabla 0.0000 \nabla \nabla \nabla \nabla 0.0000$ 


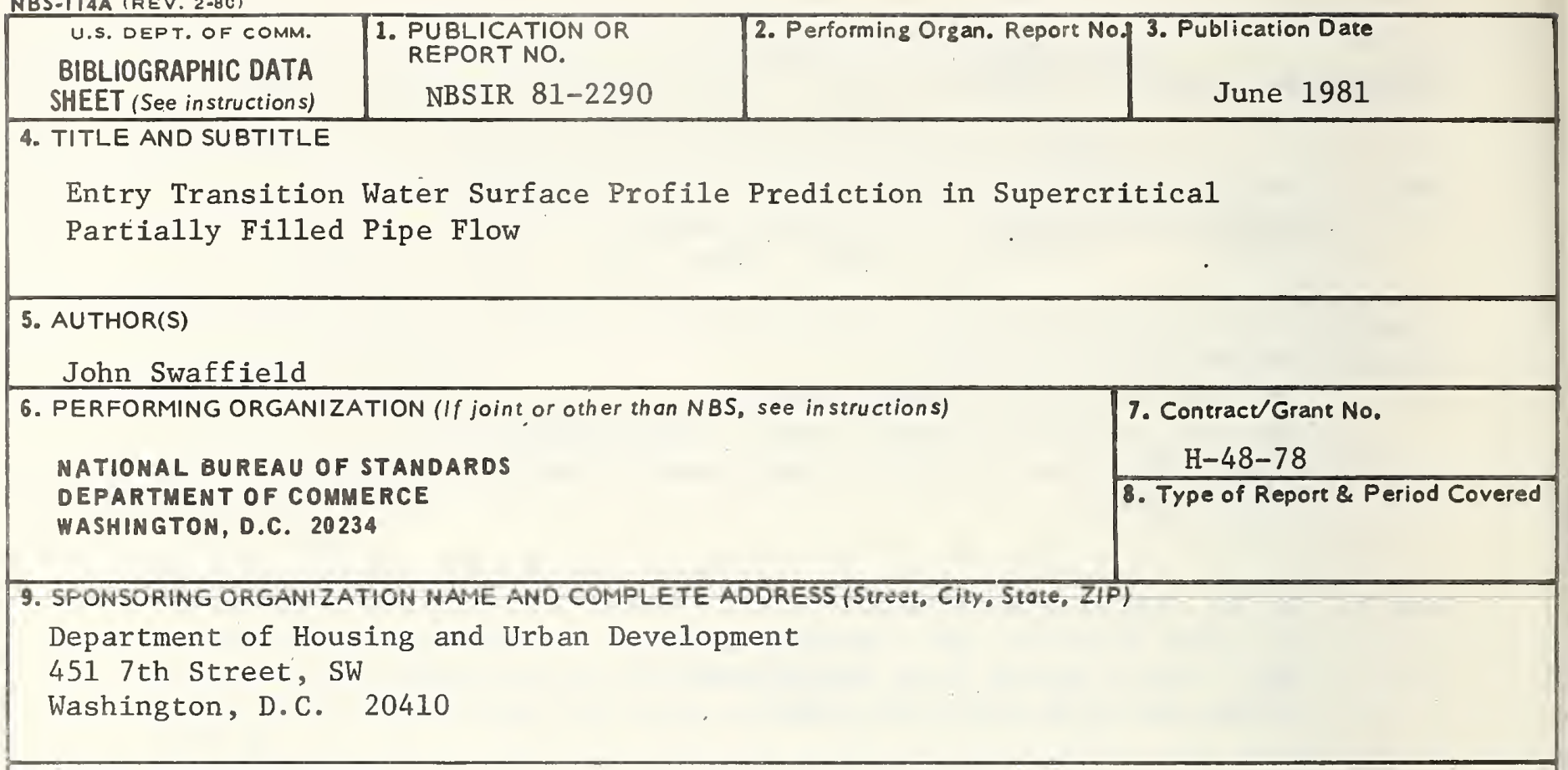

10. SUPPLEMENTARY NOTES

Document describes a computer program; SF-185, FIPS Software Summary, is attached.

11. ABSTRACT (A 200-word or less factual summary of most significant information. If document includes a s/gniflcant bibliography or literature survey, mention it here)

The criteria governing the development of steady partially filled supercritical pipe flow are presented together with the necessary techniques to determine the water surface profile in the pipe entry transition length.

The establishment of full bore flow is predicted for a range of flow rates and pipe design parameters. Based on the water surface profile calculation technique pipe length predictions are presented to avoid the air pressure fluctuations in the drainage system that result from full bore flow establishment.

Tabular data is presented to allow design decisions to be made that link pipe slope, diameter and roughness to the need to avoid full bore flow. A graphical technique is also presented that removes the necessity to interpolate from the tabular data.

The effect of entry geometry loss coefficients is included in the techniques presented.

12. KEY WORDS (Six to twelve entries; alphabetical order; capitalize only proper names; and separate key words by semicolons) Building drainage; supercritical flow; transition length; vertical stack drain entry.

13. AVAILABILITY

X Unlimited

For Official Distribution. Do Not Release to NTIS

[ Order From Superintendent of Documents, U.S. Government Printing Office, Washington, D.C. 20402.

[Xorder From National Technical Information Service (NTIS), Springfield, VA. 2216I

14. NO. OF

PRINTED PAGES

107

15. Price

$\$ 11.00$ 

